Cochrane Database of Systematic Reviews

\title{
Intrauterine administration of human chorionic gonadotropin (hCG) for subfertile women undergoing assisted reproduction (Review)
}

\author{
Craciunas L, Tsampras N, Raine-Fenning N, Coomarasamy A
}

Craciunas L, Tsampras N, Raine-Fenning N, Coomarasamy A.

Intrauterine administration of human chorionic gonadotropin (hCG) for subfertile women undergoing assisted reproduction.

Cochrane Database of Systematic Reviews 2018, Issue 10. Art. No.: CD011537.

DOI: 10.1002/14651858.CD011537.pub3. 
TABLE OF CONTENTS

HEADER 1

ABSTRACT

PLAIN LANGUAGE SUMMARY

SUMMARY OF FINDINGS

BACKGROUND

OBJECTIVES

METHODS

RESULTS

Figure 1.

Figure 2.

Figure 3.

Figure 4.

Figure 5.

Figure 6.

Figure 7.

Figure 8.

DISCUSSION

AUTHORS' CONCLUSIONS

ACKNOWLEDGEMENTS

REFERENCES

CHARACTERISTICS OF STUDIES

DATA AND ANALYSES

Analysis 1.1. Comparison 1 Intrauterine human chorionic gonadotropin (hCG) versus no hCG, Outcome 1 Live birth.

Analysis 1.2. Comparison 1 Intrauterine human chorionic gonadotropin (hCG) versus no hCG, Outcome 2 Miscarriage.

Analysis 1.3. Comparison 1 Intrauterine human chorionic gonadotropin (hCG) versus no hCG, Outcome 3 Miscarriage per clinical pregnancy.

Analysis 1.4. Comparison 1 Intrauterine human chorionic gonadotropin (hCG) versus no hCG, Outcome 4 Clinical pregnancy. ..

Analysis 1.5. Comparison 1 Intrauterine human chorionic gonadotropin (hCG) versus no hCG, Outcome 5 Complications. APPENDICES

WHAT'S NEW

HISTORY

CONTRIBUTIONS OF AUTHORS

DECLARATIONS OF INTEREST

SOURCES OF SUPPORT

DIFFERENCES BETWEEN PROTOCOL AND REVIEW

INDEX TERMS 
[Intervention Review]

\section{Intrauterine administration of human chorionic gonadotropin (hCG) for subfertile women undergoing assisted reproduction}

Laurentiu Craciunas $^{1}$, Nikolaos Tsampras ${ }^{2}$, Nick Raine-Fenning ${ }^{3}$, Arri Coomarasamy ${ }^{1}$

1Tommy's National Centre for Miscarriage Research, Institute of Metabolism and Systems Research, University of Birmingham, Birmingham, UK. ${ }^{2}$ Reproductive Medicine, St Marys Hospital, Central Manchester University Hospital NHS Trust, Manchester, UK. 3Division of Child Health, Obstetrics and Gynaecology, School of Medicine, University of Nottingham, Nottingham, UK

Contact address: Laurentiu Craciunas, Tommy's National Centre for Miscarriage Research, Institute of Metabolism and Systems Research, University of Birmingham, Birmingham, B15 2TG, UK. Icraciunas@doctors.org.uk.

Editorial group: Cochrane Gynaecology and Fertility Group.

Publication status and date: Edited (no change to conclusions), published in Issue 10, 2018.

Citation: Craciunas L, Tsampras N, Raine-Fenning N, Coomarasamy A. Intrauterine administration of human chorionic gonadotropin (hCG) for subfertile women undergoing assisted reproduction. Cochrane Database of Systematic Reviews 2018, Issue 10. Art. No.: CD011537. DOI: 10.1002/14651858.CD011537.pub3.

Copyright ( 2018 The Cochrane Collaboration. Published by John Wiley \& Sons, Ltd.

\section{A B S T R A C T}

\section{Background}

Most women undergoing assisted reproduction treatment will reach the stage of embryo transfer (ET), but the proportion of embryos that can be successfully implanted after ET has remained small since the mid-1990s. Human chorionic gonadotropin (hCG) is a hormone that is synthesised and released by the syncytiotrophoblast and has a fundamental role in embryo implantation and the early stages of pregnancy. Intrauterine administration of hCG via ET catheter during a mock procedure around the time of ET is a novel approach that has been suggested to improve the outcomes of assisted reproduction.

\section{Objectives}

To investigate whether intrauterine (intracavity) administration of hCG (IC-hCG) around the time of ET improves clinical outcomes in subfertile women undergoing assisted reproduction.

\section{Search methods}

We performed searches on 9 January 2018 using Cochrane methods.

\section{Selection criteria}

We looked for randomised controlled trials (RCTs) evaluating IC-hCG around the time of ET, irrespective of language and country of origin.

\section{Data collection and analysis}

Two review authors independently selected studies, assessed risk of bias, extracted data from studies, and attempted to contact study authors when data were missing. We performed statistical analysis using Review Manager 5. We assessed evidence quality using GRADE methods. Primary outcomes were live birth and miscarriage; secondary outcomes were clinical pregnancy rate and complications.

\section{Main results}

Seventeen RCTs investigated the effects of IC-hCG administration for 4751 subfertile women undergoing assisted reproduction. IC-hCG was administered in variable doses at different times before the ET. hCG was obtained from the urine of pregnant women or from cell cultures using recombinant DNA technology. 
Most studies (12/17) were at high risk of bias in at least one of the seven domains assessed. Common problems were unclear reporting of study methods and lack of blinding. The main limitations for evidence quality were high risk of bias and serious imprecision.

For analyses of live birth and clinical pregnancy, there was considerable heterogeneity $\left(1^{2}>75 \%\right)$ and therefore we present subgroups for dosage and stage of ET. Exploration for sources of heterogeneity revealed two key prespecified variables as important determinants: stage of ET (cleavage vs blastocyst stage) and dose of IC-hCG (<500 international units (IU) vs $\geq 500$ IU). We performed meta-analyses within subgroups defined by stage of embryo and dose of IC-hCG.

Live birth rates among women having cleavage-stage ET with an IC-hCG dose $<500$ IU compared to women having cleavage-stage ET without IC-hCG showed no benefit of the intervention and would be consistent with no substantive difference or disadvantage of indeterminate magnitude (risk ratio (RR) $0.76,95 \%$ confidence interval $(\mathrm{Cl}) 0.58$ to 1.01 ; one $\mathrm{RCT} ; 280$ participants; $I^{2}=0 \%$; very low-quality evidence). In a clinic with a live birth rate of $49 \%$ per cycle, use of IC-hCG < 500 IU would be associated with a live birth rate ranging from $28 \%$ to $50 \%$.

Results show an increase in live birth rate in the subgroup of women undergoing cleavage-stage ET with an IC-hCG dose $\geq 500$ IU compared to women having cleavage-stage ET without IC-hCG (RR 1.57, 95\% Cl 1.32 to 1.87; three RCTs; 914 participants; I $^{2}=0 \%$; moderate-quality evidence). At a clinic with a live birth rate of $27 \%$ per cycle, use of IC-hCG $\geq 500$ IU would be associated with a live birth rate ranging from $36 \%$ to $51 \%$.

Results show no substantive differences in live birth among women having blastocyst-stage ET with an IC-hCG dose $\geq 500$ IU compared to women having blastocyst-stage ET without IC-hCG (RR 0.92, 95\% Cl 0.80 to 1.04; two RCTs; 1666 participants; I I $^{2}$ 0\%; moderate-quality evidence). At a clinic with a live birth rate of $36 \%$ per cycle, use of IC-hCG $\geq 500$ IU would be associated with a live birth rate ranging from $29 \%$ to $38 \%$.

Evidence for clinical pregnancy among women having cleavage-stage ET with an IC-hCG dose $<500$ IU showed no benefit of the intervention and would be consistent with no substantive difference or disadvantage of indeterminate magnitude ( $\mathrm{RR} 0.88,95 \% \mathrm{Cl} 0.70$ to 1.10 ; one RCT; 280 participants; $I^{2}=0 \%$; very low-quality evidence).

Results show an increase in clinical pregnancy rate in the subgroup of women having cleavage-stage ET with an IC-hCG dose $\geq 500$ IU compared to women having cleavage-stage ET without IC-hCG (RR 1.49, 95\% Cl 1.32 to 1.68; 12 RCTs; 2186 participants; I $^{2}=18 \%$; moderatequality evidence).

Results show no substantive differences in clinical pregnancy among women having blastocyst-stage ET with an IC-hCG dose $\geq 500$ IU (RR $0.99,95 \% \mathrm{Cl} 0.85$ to 1.15 ; four RCTs; 2091 participants; $\mathrm{I}^{2}=42 \%$; moderate-quality evidence) compared to women having blastocyst-stage ET with no IC-hCG.

No RCTs investigated blastocyst-stage ET with an IC-hCG dose $<500$ IU.

We are uncertain whether miscarriage was influenced by intrauterine hCG administration (RR 1.04, 95\% CI 0.81 to $1.35 ; 11$ RCTs; 3927 participants; $I^{2}=0 \%$; very low-quality evidence).

Reported complications were ectopic pregnancy (four RCTs; 1073 participants; four events overall), heterotopic pregnancy (one RCT; 495 participants; one event), intrauterine death (three RCTs; 1078 participants; 22 events), and triplets (one RCT; 48 participants; three events). Events were few, and very low-quality evidence was insufficient to permit conclusions to be drawn.

\section{Authors' conclusions}

There is moderate quality evidence that women undergoing cleavage-stage transfer using an IC-hCG dose $\geq 500$ IU have an improved live birth rate. There is insufficient evidence for IC-hCG treatment for blastocyst transfer. There should be further trials with live birth as the primary outcome to identify the groups of women who would benefit the most from this intervention. There was no evidence that miscarriage was reduced following IC-hCG administration, irrespective of embryo stage at transfer or dose of IC-hCG. Events were too few to allow conclusions to be drawn with regard to other complications.

\section{PLAIN LANGUAGE SUMMARY}

The effect of administering pregnancy hormone into the womb of subfertile women undergoing assisted reproduction

\section{Review question}

Does administering pregnancy hormone into the womb of subfertile women undergoing assisted reproduction provide any benefit?

\section{Background}

Subfertility affects $15 \%$ of couples and is defined as the inability to become pregnant naturally following 12 months of regular unprotected sexual intercourse. Assisted reproduction refers to procedures involving handling of both sperm and eggs in the laboratory to create 
embryos to be transferred into the womb (embryo transfer (ET)). Administering natural or synthetic pregnancy hormone into the womb of subfertile women undergoing assisted reproduction treatment is a novel approach that might increase the chance of having a baby.

\section{Study characteristics}

We evaluated 17 studies (4751 women) comparing administration of pregnancy hormone versus no hormone. The natural or synthetic hormone was administered at variable doses at different times before ET.

\section{Key results}

Live birth rates in women having day three ET with human chorionic gonadotropin administered into the uterus (IC-hCG) at a dose $<500$ IU compared to women having day three ET without pregnancy hormone showed no benefit of the intervention and would be consistent with no substantive difference or disadvantage of indeterminate magnitude (very low-quality evidence: one study; 280 women). In a clinic with a live birth rate of $49 \%$ per cycle following day three ET, use of a pregnancy hormone dose $<500$ IU would be associated with a live birth rate varying from $28 \%$ to $50 \%$.

Live birth rate was increased in a subgroup of women having day three ET with a pregnancy hormone dose of 500 IU or greater compared to women having day three ET without pregnancy hormone (moderate-quality evidence: three studies; 914 women). At a clinic with a live birth rate of $27 \%$ per cycle, use of a pregnancy hormone dose of $500 \mathrm{IU}$ or greater would be associated with a live birth rate varying from $36 \%$ to $51 \%$.

Trial results show no substantive differences in live birth among women having day five ET with a pregnancy hormone dose of $500 \mathrm{IU}$ or greater compared to women having day five ET without pregnancy hormone (moderate-quality evidence: two studies; 1666 women). At a clinic with a live birth rate of $36 \%$ per cycle, use of a pregnancy hormone dose of $500 \mathrm{IU}$ or greater would be associated with a live birth rate varying from $29 \%$ to $38 \%$.

We are uncertain whether administration of pregnancy hormone into the womb at any dose or time affected miscarriage (very low-quality evidence: 11 studies; 3927 women).

Evidence for clinical pregnancy among women having day three ET with a pregnancy hormone dose $<500$ IU showed no benefit of the intervention and would be consistent with no substantive difference or disadvantage of indeterminate magnitude (very low-quality evidence: one study; 280 women).

The clinical pregnancy rate was increased in the subgroup of women having day three ET with a pregnancy hormone dose of 500 IU or greater compared to women having day three ET without pregnancy hormone (moderate-quality evidence: 12 studies; 2186 women).

Trial results show no substantive difference in clinical pregnancy among women having day five ET with a pregnancy hormone dose of 500 IU or greater compared to women having day five ET with no pregnancy hormone (moderate-quality evidence: four studies; 2091 women).

No randomised controlled trials (RCTs) investigated day five ET with a pregnancy hormone dose $<500 \mathrm{IU}$.

Other complications reported in the included studies were ectopic pregnancy (where the embryo develops outside the womb), heterotopic pregnancy (where embryos develop inside and outside the womb), foetal death, and triplets. Events were few, and insufficient evidence of very low quality does not permit us to determine whether there were differences between groups.

There should be further trials with live birth as the primary outcome to identify the groups of women who would benefit the most from this intervention.

\section{Quality of the evidence}

Evidence quality varied from very low to moderate depending on the outcome. The main limitations for the overall quality of the evidence were high risk of bias and serious imprecision. 


\section{SUMMARY OF FINDINGS}

\section{Summary of findings for the main comparison. Intrauterine administration of hCG for women undergoing assisted reproduction}

\section{Intrauterine administration of hCG for women undergoing assisted reproduction}

Patient or population: subfertile women undergoing assisted reproduction

Setting: assisted reproduction units

Intervention: intrauterine human chorionic gonadotropin (hCG)

Comparison: no intrauterine hCG

\begin{tabular}{|c|c|c|c|c|c|}
\hline \multirow[t]{2}{*}{ Outcomes } & \multicolumn{2}{|c|}{ Anticipated absolute effects* $(95 \% \mathrm{Cl})$} & \multirow{2}{*}{$\begin{array}{l}\text { Relative effect } \\
(95 \% \mathrm{Cl})\end{array}$} & \multirow{2}{*}{$\begin{array}{l}\text { No. of partici- } \\
\text { pants } \\
\text { (studies) }\end{array}$} & \multirow{2}{*}{$\begin{array}{l}\text { Certainty of the } \\
\text { evidence } \\
\text { (GRADE) }\end{array}$} \\
\hline & Risk with no hCG & $\begin{array}{l}\text { Risk with intrauterine human } \\
\text { chorionic gonadotropin (hCG) }\end{array}$ & & & \\
\hline \multirow{2}{*}{$\begin{array}{l}\text { Live birth } \\
\text { Cleavage stage: } \mathrm{hCG}<500 \mathrm{IU} \\
\text { Follow-up: mean } 40 \text { weeks }\end{array}$} & 495 per 1000 & $\begin{array}{l}376 \text { per } 1000 \\
\text { ( } 287 \text { to } 500)\end{array}$ & $\begin{array}{l}\text { RR } 0.76 \\
\text { (0.58 to } 1.01)\end{array}$ & $\begin{array}{l}280 \\
(1 \mathrm{RCT})\end{array}$ & $\begin{array}{l}\oplus \ominus \ominus \ominus \\
\text { VERY LOWa,b }\end{array}$ \\
\hline & 273 per 1000 & $\begin{array}{l}428 \text { per } 1000 \\
(360 \text { to } 510)\end{array}$ & $\begin{array}{l}\text { RR } 1.57 \\
\text { (1.32 to } 1.87)\end{array}$ & $\begin{array}{l}914 \\
\text { (3 RCTs) }\end{array}$ & $\begin{array}{l}\oplus \oplus \oplus \ominus \\
\text { MODERATEC }\end{array}$ \\
\hline $\begin{array}{l}\text { Blastocyst stage: } \mathrm{hCG} \geq 500 \mathrm{IU} \\
\text { Follow-up: mean } 40 \text { weeks }\end{array}$ & 369 per 1000 & $\begin{array}{l}340 \text { per } 1000 \\
\text { (296 to } 384 \text { ) }\end{array}$ & $\begin{array}{l}\text { RR } 0.92 \\
\text { (0.80 to } 1.04)\end{array}$ & $\begin{array}{l}1666 \\
\text { (2 RCTs) }\end{array}$ & $\begin{array}{l}\oplus \oplus \oplus \ominus \\
\text { MODERATEC }\end{array}$ \\
\hline $\begin{array}{l}\text { Miscarriage } \\
\text { Follow-up: mean } 40 \text { weeks }\end{array}$ & 58 per 1000 & $\begin{array}{l}60 \text { per } 1000 \\
(47 \text { to } 78)\end{array}$ & $\begin{array}{l}\text { RR } 1.04 \\
\text { (0.81 to } 1.35)\end{array}$ & $\begin{array}{l}3927 \\
\text { (11 RCTs) }\end{array}$ & $\begin{array}{l}\oplus \ominus \ominus \ominus \\
\text { VERY LOWc,d }\end{array}$ \\
\hline $\begin{array}{l}\text { Clinical pregnancy Cleavage stage: } \\
\text { hCG < } 500 \text { IU } \\
\text { Follow-up: mean } 12 \text { weeks }\end{array}$ & 579 per 1000 & $\begin{array}{l}509 \text { per } 1000 \\
\text { (405 to } 637 \text { ) }\end{array}$ & $\begin{array}{l}\text { RR } 0.88 \\
(0.70 \text { to } 1.10)\end{array}$ & $\begin{array}{l}280 \\
(1 \mathrm{RCT})\end{array}$ & $\begin{array}{l}\oplus \ominus \ominus \ominus \\
\text { VERY LOWa,d }\end{array}$ \\
\hline $\begin{array}{l}\text { Cleavage stage: } \mathrm{hCG} \geq 500 \mathrm{IU} \\
\text { Follow-up: mean } 12 \text { weeks }\end{array}$ & 307 per 1000 & $\begin{array}{l}458 \text { per } 1000 \\
\text { (406 to } 517 \text { ) }\end{array}$ & $\begin{array}{l}\text { RR } 1.49 \\
\text { (1.32 to } 1.68)\end{array}$ & $\begin{array}{l}2186 \\
(12 \mathrm{RCTs})\end{array}$ & $\begin{array}{l}\oplus \oplus \oplus \ominus \\
\text { MODERATEC }\end{array}$ \\
\hline $\begin{array}{l}\text { Blastocyst stage: } \mathrm{hCG} \geq 500 \text { IU } \\
\text { Follow-up: mean } 12 \text { weeks }\end{array}$ & 422 per 1000 & $\begin{array}{l}418 \text { per } 1000 \\
\text { (359 to } 485)\end{array}$ & $\begin{array}{l}\text { RR } 0.99 \\
\text { (0.85 to } 1.15)\end{array}$ & $\begin{array}{l}2091 \\
\text { (4 RCTs) }\end{array}$ & $\begin{array}{l}\oplus \oplus \oplus \ominus \\
\text { MODERATEC }\end{array}$ \\
\hline $\begin{array}{l}\text { Complications } \\
\text { Follow-up: mean } 40 \text { weeks }\end{array}$ & $\begin{array}{l}\text { Other complicatio } \\
\text { ectopic pregnancy } \\
\text { erotopic pregnanc } \\
\text { death (3 RCTs; } \mathrm{N}=\end{array}$ & $\begin{array}{l}\text { ted in the included studies were } \\
\mathrm{N}=1073 ; 4 \text { events overall), het- } \\
\mathrm{N}=495 ; 1 \text { event), intrauterine } \\
\text { events), and triplets ( } 1 \mathrm{RCT} ; \mathrm{N}=48\end{array}$ & - & $\begin{array}{l}1764 \\
\text { (7 RCTs) }\end{array}$ & $\begin{array}{l}\oplus \ominus \ominus \ominus \\
\text { VERY LOWc,d }\end{array}$ \\
\hline
\end{tabular}

1764 
${ }^{\star}$ The risk in the intervention group (and its $95 \%$ confidence interval) is based on the assumed risk in the comparison group and the relative effect of the intervention (and its $95 \% \mathrm{Cl}$ ).

$\mathrm{Cl}$ : confidence interval; hCG: human chorionic gonadotropin; RCT: randomised controlled trial; RR: risk ratio.

\section{GRADE Working Group grades of evidence.}

High certainty: we are very confident that the true effect lies close to that of the estimate of the effect.

Moderate certainty: we are moderately confident in the effect estimate: the true effect is likely to be close to the estimate of the effect, but there is a possibility that it is substantially different.

Low certainty: our confidence in the effect estimate is limited: the true effect may be substantially different from the estimate of the effect.

Very low certainty: we have very little confidence in the effect estimate: the true effect is likely to be substantially different from the estimate of effect.

${ }^{a}$ Downgraded two levels for very serious risk of bias: lack of blinding of participants and personnel, no clear description of allocation concealment, and premature termination of the study following interim analysis.

bDowngraded one level for serious imprecision: total events were fewer than 300.

cDowngraded one level for serious risk of bias: lack of blinding of participants and personnel, no allocation concealment.

dDowngraded two levels for very serious imprecision: total number of events was less than 300 , and $95 \%$ confidence interval around the pooled effect includes both no effect and appreciable benefit or appreciable harm. 


\section{B A C K G R O U N D}

\section{Description of the condition}

Subfertility is defined as the inability of a couple to conceive spontaneously following 12 months of regular unprotected sexual intercourse. It is estimated that $15 \%$ of couples are affected by subfertility of different causes (female factor, male factor, unexplained). Assisted reproduction refers to procedures involving the in vitro (in a laboratory dish) handling of both human gametes (sperm and eggs) with the objective of establishing a pregnancy (Zegers-Hochschild 2009). The most vulnerable step of assisted reproduction is the embryo transfer (ET), as it involves a radical change in the embryo's environment, which makes it prone to demise (Schoolcraft 2001). Most women undergoing assisted reproduction treatment will reach the stage of ET owing to important improvements in ovarian stimulation protocols and laboratory technology, but the proportion of embryos that successfully implant following ET has remained small (less than one-third) since the mid-1990s (Kupka 2014).

The process of implantation involves a reciprocal interaction between the embryo and the endometrium, culminating in a small reception-ready phase of the endometrium, during which implantation can occur. This interaction is dependent on the temporal differentiation of endometrial cells to attain uterine receptivity. Implantation failure is thought to occur as a consequence of impairment of the embryo developmental potential or impairment of uterine receptivity, or both, and the embryo-uterine dialogue (Diedrich 2007).

Many interventions have been attempted with varying degrees of success before ET (endometrial injury (Nastri 2012), dummy ET (Mansour 1990), endometrial preparation (Derks 2009), periimplantation (heparin (Akhtar 2013), aspirin (Siristatidis 2016)), during ET (ultrasound guidance (Brown 2010), removal of cervical mucus (Craciunas 2014)), and after ET (fibrin sealant, bed rest (Abou-Setta 2014)) to optimise the embryo-endometrial interaction and improve outcomes.

\section{Description of the intervention}

Human chorionic gonadotropin ( $\mathrm{hCG}$ ) is a hormone that is synthesised and released by the syncytiotrophoblast. It stimulates ovarian production of progesterone during the first trimester of pregnancy. Intrauterine administration of synthetic or natural hCG around the time of ET is a novel approach that has been suggested to improve the outcomes of assisted reproduction treatment based on the fundamental role of hCG in embryo implantation and the early stages of pregnancy (Cole 2010). The intervention involves intrauterine administration of hCG via an ET catheter during a mock procedure (a trial of the actual ET without using an embryo, performed to assess the difficulty of the ET) using the lowest volume of medium before the conventional ET. The hCG can be released at different points inside the uterine cavity (close to the internal cervical os, mid-cavity, or near the fundus) within minutes, hours, or days before the actual ET. hCG sources for medical treatments include extraction from the urine of pregnant women (natural) or from cell cultures using recombinant DNA technology (rhCG).

\section{How the intervention might work}

The hCG may promote peritrophoblastic immune tolerance, which facilitates trophoblast invasion by inducing an increase in endometrial T-cell apoptosis (Kayisli 2003). It also supports trophoblast apposition (the first stage of implantation - loose alignment of the trophoblast to the decidua) and adhesion (second stage of implantation - closer attachment of the trophoblast to the decidua) to the endometrium by regulating proteins involved in implantation (Racicot 2014). Intrauterine injection of urinary hCG alters endometrial secretory parameters (Licht 1998), and cell proliferation and migration are increased in the presence of hCG (Bourdiec 2013).

\section{Why it is important to do this review}

Subfertility affects a relatively large proportion of couples, and assisted reproduction treatments remain costly and stressful. All effort should be directed towards increasing the success rate of infertility treatments, and primary research should be translated into clinical practice in an efficient and timely manner. Intrauterine administration of hCG around the time of ET has the potential to improve the outcomes of assisted reproduction treatments; randomised and non-randomised trials have reported varying results (Mansour 2011; Rebolloso 2013).

Previous meta-analyses assessed the efficacy of intrauterine injection of hCG before ET in assisted reproductive cycles, but improvements could be made to the methods of analysis (Dieamant 2016; Osman 2016; Ye 2015). Different studies have evaluated variable circumstances of intrauterine hCG administration in terms of stage of the embryo at transfer (cleavage vs blastocyst), source of hCG (urine vs recombinant), dose of hCG, embryo processing (fresh vs frozen/thawed), and number of embryos transferred, leading to real uncertainties about the role of the intervention. The previous version of this review reported promising outcomes for cleavage-stage ET following intrauterine injection of hCG at a dose of 500 IU or more (Craciunas 2016), but the evidence was weak and newly published randomised controlled trials may have altered our confidence in the results.

\section{O B JECTIVES}

To investigate whether intrauterine (intracavity) administration of hCG (IC-hCG) around the time of ET improves clinical outcomes in subfertile women undergoing assisted reproduction.

\section{METHODS}

\section{Criteria for considering studies for this review}

\section{Types of studies}

We included in this review all randomised controlled trials (RCTs) evaluating intrauterine (intracavity) administration of hCG (IC-hCG) around the time of ET, irrespective of language and country of origin. We planned to include only data from the first phase of crossover RCTs in meta-analyses.

\section{Types of participants}

We included subfertile women undergoing in vitro fertilisation (IVF)/intracytoplasmic sperm injection (ICSI) followed by ET. 


\section{Types of interventions}

RCTs comparing intrauterine administration of hCG around the time of ET versus any other active intervention, no intervention, or placebo were eligible for inclusion.

\section{Types of outcome measures}

\section{Primary outcomes}

- Live birth (delivery of a live foetus after 24 completed weeks of gestation) rate per woman or couple randomised

- Miscarriage (loss of pregnancy before 24 completed weeks of gestation) rate per woman or couple randomised

\section{Secondary outcomes}

- Clinical pregnancy (presence of a gestational sac on ultrasound scan) rate per woman or couple randomised

- Complication rate per woman or couple randomised, including ectopic pregnancy, intrauterine growth restriction, foetal or congenital defects, pelvic infection, or other adverse events, reported as an overall complication rate or as individual outcomes, or both (as reported by individual studies)

\section{Search methods for identification of studies}

We sought all published and unpublished RCTs of intrauterine hCG administration around the time of ET in consultation with the Cochrane Gynaecology and Fertility Group Information Specialist. Search dates ranged from inception of the databases to 9 January 2018 , and we applied no language restrictions.

\section{Electronic searches}

We searched the following.

- Cochrane Gynaecology and Fertility Group Specialised Register (searched 9 January 2018) (PROCITE platform) (Appendix 1).

- Cochrane Central Register of Controlled Trials (CENTRAL) in the Cochrane Library (via the CENTRAL Register of Studies Online (CRSO)) (searched 9 January 2018) (Web platform) (Appendix 2).

- MEDLINE (searched from 1946 to 9 January 2018) (OVID platform) (Appendix 3).

- Embase (searched from 1980 to 9 January 2018) (OVID platform) (Appendix 4).

- PsycINFO (searched from 1806 to 9 January 2018) (OVID platform) (Appendix 5).

- Cumulative Index to Nursing and Allied Health Literature (CINAHL) (searched from 1961 to 9 January 2018) (EBSCO platform) (Appendix 6).

We combined the MEDLINE search with the Cochrane highly sensitive search strategy for identifying RCTs, which appears in the Cochrane Handbook for Systematic Reviews of Interventions (Higgins 2011; Chapter 6, Section 6.4.11). We combined the Embase and CINAHL searches with trial filters developed by the Scottish Intercollegiate Guidelines Network (SIGN) (www.sign.ac.uk/methodology/filters.html\#random).

We also searched the World Health Organization International Clinical Trials Registry Platform (apps.who.int/trialsearch/ Default.aspx) and ClinicalTrials.gov for ongoing and registered trials. We searched OpenGrey (www.opengrey.eu/) and Google Scholar (scholar.google.co.uk/) for grey literature. We handsearched abstracts published following major conferences (e.g. the American Society for Reproductive Medicine (ASRM), European Society of Human Reproduction and Embryology (ESHRE)) held in the last five years to find additional studies not yet published in full.

\section{Searching other resources}

We screened the reference lists of all included studies and relevant reviews to identify further articles for possible inclusion.

\section{Data collection and analysis}

We used Review Manager 5 for input of data and statistical analysis (RevMan 2014), in accordance with the Cochrane Handbook for Systematic Reviews of Interventions (Higgins 2011).

\section{Selection of studies}

Two review authors (LC and NT) independently screened the title, abstract, and keywords for each publication to exclude studies that were irrelevant for the objective of this review. We retrieved the remaining publications in full text, and the same two review authors appraised them independently to identify RCTs that were suitable for inclusion. We encountered no disagreements related to study eligibility and documented the selection process with a PRISMA flow chart.

\section{Data extraction and management}

Two review authors (LC and NT) independently extracted data using a pre-designed and pilot-tested data extraction form. For studies with multiple publications, we used the main RCT report as the reference, and we supplemented it with additional data from secondary publications. We attempted to contact study authors when published data were insufficient. We encountered no disagreements. One review author (LC) entered data into Review Manager 5 (RevMan 2014), and a second review author (NT) checked entered data against the data extraction form.

\section{Assessment of risk of bias in included studies}

We used the Cochrane 'Risk of bias' assessment tool to assess the included studies for selection, performance, detection, attrition, reporting, and other biases. We encountered no disagreements. We included the 'Risk of bias' table in the Characteristics of included studies table, describing the judgements in detail.

\section{Measures of treatment effect}

All outcomes were dichotomous. We calculated Mantel-Haenszel risk ratios (RRs) with 95\% confidence intervals (Cls) using the numbers of events in the intervention and control groups of each study. For outcomes with event rates below $1 \%$, we used the Peto one-step odds ratio (OR) method to calculate the combined outcome with $95 \% \mathrm{Cl}$.

\section{Unit of analysis issues}

We performed analysis per randomised woman or couple for live birth, clinical pregnancy, miscarriage, and complication rates. We counted multiple live births (twins, triplets) as a single live birth event. We performed a secondary analysis for miscarriage per clinical pregnancy to broaden our understanding of the treatment effect. 
If a study included multiple treatment arms based on hCG dose, we planned to split the control group proportionately with the experimental groups to avoid analysing control participants in duplicate.

\section{Dealing with missing data}

We attempted to contact authors of the RCTs to obtain missing data so we could perform analyses on an intention-to-treat basis. In the case of unobtainable data, we planned to undertake imputation of individual values for the live birth rate only. We assumed that live births had not occurred in participants without a reported outcome. For other outcomes, we analysed only available data.

\section{Assessment of heterogeneity}

We identified heterogeneity by visually inspecting forest plots and by using a standard $\mathrm{Chi}^{2}$ test with significance set at $\mathrm{P}<0.1$. We used the $I^{2}$ statistic to estimate total variation across RCTs that was due to heterogeneity, when $\mathrm{I}^{2}$ greater than $50 \%$ indicated substantial heterogeneity.

\section{Assessment of reporting biases}

We conducted a comprehensive search to minimise the potential impact of publication bias and other reporting biases. We planned to use a funnel plot to explore the possibility of small-study effects when the number of included RCTs exceeded 10.

\section{Data synthesis}

We combined the data from similar RCTs comparing similar treatments using a random-effects model. We displayed an increase in the odds of an outcome to the right of the centre line and a decrease in the odds of an outcome to the left of the centre line. For comparisons that showed considerable clinical, methodological, or statistical heterogeneity $\left(I^{2}>75 \%\right)$, we did not combine results of RCTs in a meta-analysis. When data were incomplete and could not be presented in the analyses, we reported available data in narrative form.

\section{Subgroup analysis and investigation of heterogeneity}

When data were available, we conducted subgroup analyses to investigate the efficacy of intrauterine hCG administration around the time of ET depending on:

- $\quad$ stage of the embryo at transfer (cleavage vs blastocyst);

- source of intracavity hCG (IC-hCG) (urine vs recombinant);

- embryo processing (fresh vs frozen/thawed); and

- number of embryos transferred.

If we detected substantial heterogeneity, we explored possible explanations in sensitivity analyses. Factors considered included treatment indication, age of the women, ovarian stimulation protocol, response to ovarian stimulation, timing of IC-hCG administration, IC-hCG dose and volume of infused medium, method of IC-hCG administration (i.e. type of catheter), embryo quality, endometrial thickness, source of oocytes (i.e. donated, own), and ET difficulty. We took any statistical heterogeneity into account when interpreting the results, especially if we noted variation in the direction of effect.

\section{Sensitivity analysis}

We performed sensitivity analysis to examine the stability and robustness of results for the primary outcomes in relation to the following eligibility and analysis factors.

- Inclusion of RCTs without high risk of bias in one or more domains.

- Inclusion of RCTs published as full text.

- Use of a fixed-effect model.

- Calculation of OR.

\section{Overall quality of the body of evidence - 'Summary of findings' table}

Two review authors working independently (LC and NT) prepared a 'Summary of findings' table using GRADEpro software and comparing hCG versus no hCG (GRADEpro 2015). We resolved disagreements by discussion. In this table, we evaluated the overall quality of the body of evidence for the main review outcomes (live birth rate, miscarriage, clinical pregnancy rate, and complications) using GRADE criteria (study limitations (i.e. risk of bias), consistency of effect, imprecision, indirectness, and publication bias) (GRADE 2013). We justified, documented, and incorporated judgements about evidence quality (high, moderate, low, or very low) into reporting of results for each outcome.

\section{RES U L T S}

\section{Description of studies}

See Characteristics of included studies; Characteristics of excluded studies; Characteristics of studies awaiting classification; and Characteristics of ongoing studies tables.

\section{Results of the search}

We performed the latest systematic search on 9 January 2018, and we identified 352 publications (14 from CINAHL, 91 from CENTRAL, 133 from EMBASE, 41 from CGFG, 58 from MEDLINE, 2 from PsychINFO, and 13 from other sources).

In this updated review, we have included 17 studies (12 in the previous version), excluded 13 studies (six in the previous version), and identified two studies awaiting classification and five ongoing studies. See Figure 1 for detailed search results. 
Figure 1. Study flow diagram.

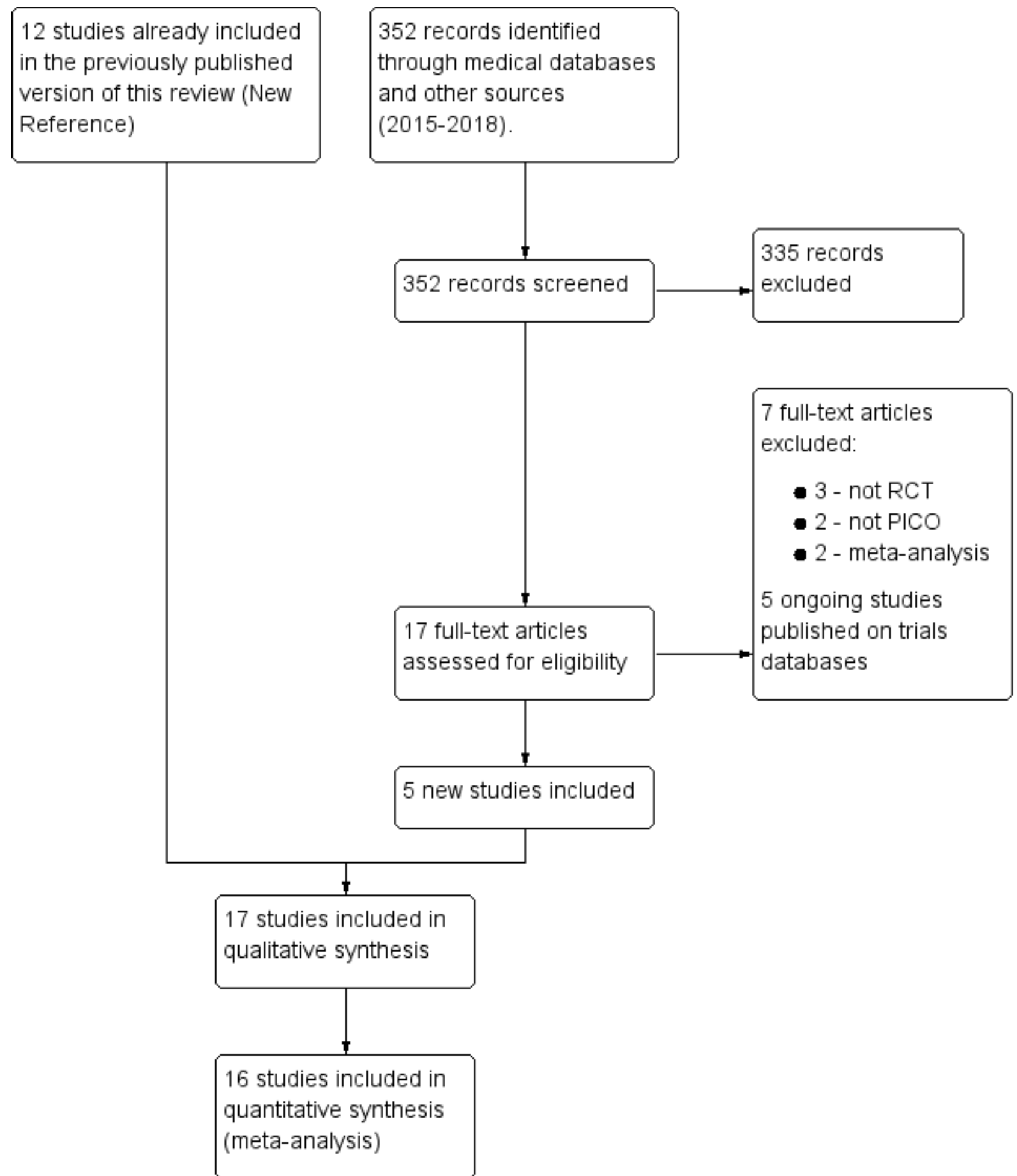

\section{Included studies}

\section{Types of studies}

All 17 included studies were parallel-arm RCTs. One study had two experimental arms (IC-hCG 500 IU vs IC-hCG 1000 IU vs control) (Dehghani Firouzabadi 2016), one study had two phases with three experimental arms (phase one: IC-hCG 100 IU vs IC-hCG 200 IU vs control; and phase two: IC-hCG 500 IU vs control) (Mansour 2011), and one study had two experimental arms using two different timings (IC-hCG 500 IU vs control two days before ET; IC-hCG 500 IU vs control on the day of ET) (Wirleitner 2015a).

Researchers performed randomisation at different times during treatment. Five studies randomised participants before the start 
of their treatment cycle (Dehghani Firouzabadi 2016; Hong 2014; Mansour 2011; Santibañez 2014; Singh 2014), two studies randomised participants on the day of oocyte retrieval (Navali 2016; Wirleitner 2015a), four studies randomised participants on the day of embryo transfer (Aaleyasin 2015; Cambiaghi 2013; Hosseini 2016; Huang 2016), and the remaining six studies provided insufficient details about the timing of randomisation (Eskandar 2016; Kokkali 2014; Leao 2013; Mostajeran 2017; Wirleitner 2015b; Zarei 2014).

Eleven studies were published as full-text articles (Aaleyasin 2015; Dehghani Firouzabadi 2016; Hong 2014; Hosseini 2016; Huang 2016; Mansour 2011; Mostajeran 2017; Navali 2016; Santibañez 2014; Wirleitner 2015a; Zarei 2014), and six studies were published as abstracts (Cambiaghi 2013; Eskandar 2016; Kokkali 2014; Leao 2013; Singh 2014; Wirleitner 2015b).

Ten studies did not report funding (Aaleyasin 2015; Cambiaghi 2013; Dehghani Firouzabadi 2016; Eskandar 2016; Hong 2014; Hosseini 2016; Huang 2016; Leao 2013; Mostajeran 2017; Wirleitner 2015a), and seven studies reported internal funding (Kokkali 2014; Mansour 2011; Navali 2016; Santibañez 2014; Singh 2014; Wirleitner 2015b; Zarei 2014). No studies reported external funding.

\section{Participants}

Participants were couples/women recruited before undergoing assisted reproductive treatment for different subfertility causes. The number of participants varied between 36 in Leao 2013 and 1186 in Wirleitner 2015a. The studies were conducted in the USA, Austria, Greece, Iran, China, Saudi Arabia, Brazil, Egypt, Mexico, and India.

\section{Interventions}

Most studies compared intrauterine administration of urine hCG $500 \mathrm{IU}$ versus controls. One study had two additional arms with lower doses (IC-hCG 100 and 200 IU) (Mansour 2011). One study had an additional arm with a higher dose (IC-hCG 1000 IU) (Dehghani Firouzabadi 2016). One study used 1000 IU (Huang 2016), and another study used $700 \mathrm{IU}$ (Mostajeran 2017). One study used rhCG $250 \mu \mathrm{g}$ (equivalent of $6500 \mathrm{IU}$ ) (Zarei 2014), and another study used intracavity rhCG (IC-rhCG) $40 \mu \mathrm{L}$ (equivalent to $500 \mathrm{IU}$ ) (Singh 2014).

Twelve studies administered IC-hCG within minutes before ET (Aaleyasin 2015; Dehghani Firouzabadi 2016; Eskandar 2016; Hong 2014; Hosseini 2016; Kokkali 2014; Mansour 2011; Mostajeran 2017; Santibañez 2014; Singh 2014; Wirleitner 2015b; Zarei 2014), ranging from less than three minutes in Hong 2014 up to 12 minutes in Zarei 2014. Two studies administered IC-hCG six hours before ET (Cambiaghi 2013; Leao 2013). One study had four groups (two experimental and two controls) at two different timings (two days before ET and three minutes before ET) (Wirleitner 2015a). One study administered IC-hCG three days before ET (Huang 2016).
Another study administered IC-hCG at the time of oocyte retrieval (Navali 2016).

For control groups, seven studies administered the same volume of transfer media (Hong 2014), culture media (Aaleyasin 2015; Singh 2014; Wirleitner 2015a; Wirleitner 2015b), or normal saline (Navali 2016; Zarei 2014), all without hCG, and 10 studies did not administer anything before ET (Cambiaghi 2013; Dehghani Firouzabadi 2016; Eskandar 2016; Hosseini 2016; Huang 2016; Kokkali 2014; Leao 2013; Mansour 2011; Mostajeran 2017; Santibañez 2014).

\section{Outcomes}

Eleven studies reported on one of our predefined primary outcomes: Aaleyasin 2015, Mansour 2011, Singh 2014, Wirleitner 2015a, and Wirleitner 2015b reported on live birth; and Aaleyasin 2015, Dehghani Firouzabadi 2016, Hong 2014, Hosseini 2016, Huang 2016, Mansour 2011, Navali 2016, Singh 2014, Wirleitner 2015a, Wirleitner 2015b, and Zarei 2014 reported on miscarriage.

Seventeen studies reported on one of our predefined secondary outcomes: Aaleyasin 2015, Cambiaghi 2013, Dehghani Firouzabadi 2016, Eskandar 2016, Hong 2014, Hosseini 2016, Huang 2016, Kokkali 2014, Leao 2013, Mansour 2011, Mostajeran 2017, Navali 2016, Santibañez 2014, Singh 2014, Wirleitner 2015a, Wirleitner 2015b, and Zarei 2014 reported on clinical pregnancy; and Aaleyasin 2015, Dehghani Firouzabadi 2016, Hosseini 2016, Mansour 2011, Navali 2016, Santibañez 2014, and Zarei 2014 reported on complications.

\section{Studies awaiting classification}

Two studies await classification (Badehnoosh 2014; Bhat 2014). These studies reported interim outcomes (implantation rate and fertilisation rate), and it is unclear whether they also collected data on clinical outcomes that might be relevant to our review. We emailed the authors of these studies in February 2016 and January 2018 to ask for more information on the methods and outcome measures of their studies.

\section{Excluded studies}

We excluded 13 studies owing to retrospective design (Huang 2017; Jeong 2013; Kanter 2017), non-randomisation (Li 2013; Rebolloso 2013; Riboldi 2013, Volovsky 2016), not meeting the PICO (Giuliani 2015; Strug 2016), and performing a meta-analysis (Dieamant 2016; Osman 2016; Ye 2015). One study was previously published as an abstract (Janati 2013); this has now been replaced by its full manuscript publication (Dehghani Firouzabadi 2016).

\section{Risk of bias in included studies}

Figure 2 shows the 'Risk of bias' graph, and Figure 3 shows the 'Risk of bias' summary. See the Characteristics of included studies table for rationales behind each judgement. 
Figure 2. Risk of bias graph: review authors' judgements about each risk of bias item presented as percentages across all included studies.

Random sequence generation (selection bias)

Allocation concealment (selection bias)

Blinding of participants and personnel (performance bias)

Blinding of outcome assessment (detection bias)

Incomplete outcome data (attrition bias)

Selective reporting (reporting bias)

Other bias

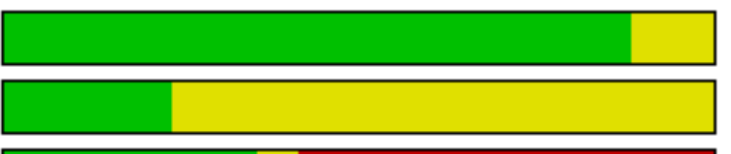

L
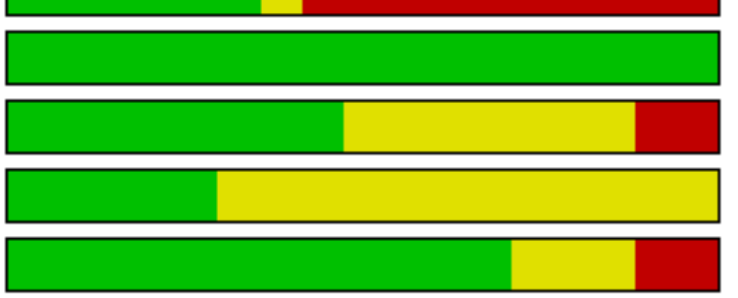

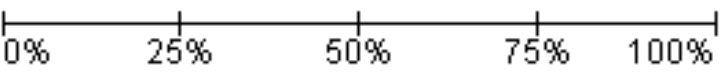


Figure 3. Risk of bias summary: review authors' judgements about each risk of bias item for each included study.

\begin{tabular}{|c|c|c|c|c|c|c|c|}
\hline & 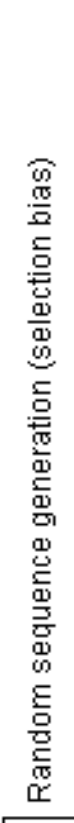 & 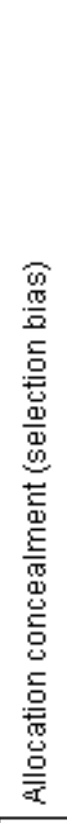 & 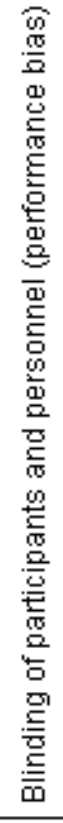 & 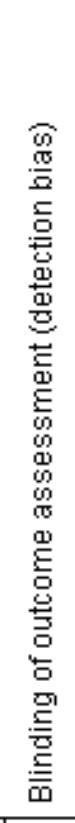 & 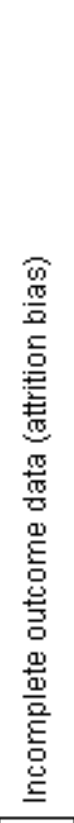 & 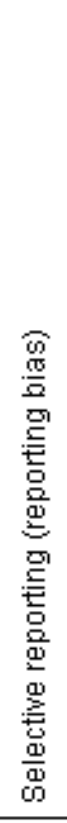 & 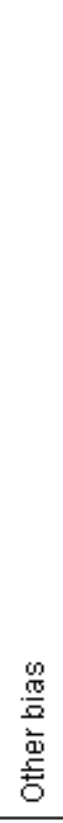 \\
\hline Aaleyasin 2015 & $\odot$ & $\oplus$ & $\oplus$ & $\odot$ & $\odot$ & $\oplus$ & $\oplus$ \\
\hline Cambiaghi 2013 & $\odot$ & $?$ & $\odot$ & $\odot$ & $?$ & $?$ & $?$ \\
\hline Dehghani Firouzabadi 2016 & + & $?$ & - & $\odot$ & $\odot$ & $?$ & $\oplus$ \\
\hline Eskandar 2016 & $\bullet$ & $?$ & - & $\odot$ & $?$ & $?$ & + \\
\hline Hong 2014 & + & $\oplus$ & $\oplus$ & $\odot$ & $\oplus$ & $?$ & $?$ \\
\hline Hosseini 2016 & $\odot$ & $?$ & - & $\odot$ & $\odot$ & $?$ & + \\
\hline Huang 2016 & $\odot$ & ? & ? & + & + & ? & + \\
\hline Kokkali 2014 & $\odot$ & $\oplus$ & - & $\odot$ & $?$ & $?$ & $?$ \\
\hline Leao 2013 & $?$ & $?$ & - & $\odot$ & $?$ & $?$ & $\odot$ \\
\hline Mansour 2011 & + & $?$ & $\odot$ & $\odot$ & $?$ & + & - \\
\hline Mostajeran 2017 & $\odot$ & $?$ & $\oplus$ & + & $?$ & $?$ & + \\
\hline Navali 2016 & $\odot$ & $\odot$ & + & $\odot$ & $\odot$ & $?$ & + \\
\hline Santibañez 2014 & $\odot$ & ? & O & + & + & ? & + \\
\hline Singh 2014 & $\odot$ & $?$ & - & $\odot$ & $\odot$ & $\odot$ & + \\
\hline Wirleitner 2015a & $\odot$ & $?$ & $\odot$ & $\odot$ & $?$ & $\oplus$ & $\oplus$ \\
\hline Wirleitner 2015b & $?$ & $?$ & + & $\odot$ & $\odot$ & + & + \\
\hline Zarei 2014 & $\odot$ & $?$ & + & 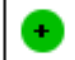 & $\odot$ & $?$ & + \\
\hline
\end{tabular}




\section{Allocation}

\section{Sequence generation}

All included studies were RCTs. The randomisation technique was adequate in 15 studies (Aaleyasin 2015; Cambiaghi 2013; Dehghani Firouzabadi 2016; Eskandar 2016; Hong 2014; Hosseini 2016; Huang 2016; Kokkali 2014; Mansour 2011; Mostajeran 2017; Navali 2016; Santibañez 2014; Singh 2014; Wirleitner 2015a; Zarei 2014), which we classified at low risk of bias. Two studies lacked an adequate description of randomisation, and we classified them at unclear risk of bias (Leao 2013; Wirleitner 2015b).

\section{Allocation concealment}

Four studies mentioned adequate allocation concealment, and we classified them at low risk of bias (Aaleyasin 2015; Hong 2014; Kokkali 2014; Navali 2016). Thirteen studies lacked a description of methods of allocation concealment, and we classified them at unclear risk of bias (Cambiaghi 2013; Dehghani Firouzabadi 2016; Eskandar 2016; Hosseini 2016; Huang 2016; Leao 2013; Mansour 2011; Mostajeran 2017; Santibañez 2014; Singh 2014; Wirleitner 2015a; Wirleitner 2015b; Zarei 2014).

\section{Blinding}

Six studies documented blinding of participants or personnel (or both), and we classified them at low risk of bias (Aaleyasin 2015; Hong 2014; Mostajeran 2017; Navali 2016; Wirleitner 2015b; Zarei 2014). One study was mentioned to be single-blinded, but it was not clear who was blinded; hence, we classified it as having unclear risk of bias (Huang 2016). We classified the remaining studies at high risk of bias (Cambiaghi 2013; Dehghani Firouzabadi 2016; Eskandar 2016; Hosseini 2016; Kokkali 2014; Leao 2013; Mansour 2011; Santibañez 2014; Singh 2014; Wirleitner 2015a).

The outcome measurement was not likely to be influenced by lack of blinding; hence, we classified all studies at low risk of bias.

\section{Incomplete outcome data}

Eight studies followed up all participants and reported the results adequately (Aaleyasin 2015; Dehghani Firouzabadi 2016; Hong 2014; Hosseini 2016; Huang 2016; Santibañez 2014; Singh 2014; Wirleitner 2015b). We classified these studies at low risk of bias. We classified seven studies at unclear risk of bias (Cambiaghi 2013; Eskandar 2016; Kokkali 2014; Leao 2013; Mansour 2011; Mostajeran 2017; Wirleitner 2015a). Two studies reported large numbers of participants lost to follow-up, and we classified them at high risk of attrition bias (Navali 2016; Zarei 2014).

\section{Selective reporting}

Five studies reported on all relevant outcomes, and we classified them at low risk of bias (Aaleyasin 2015; Mansour 2011; Singh 2014; Wirleitner 2015a; Wirleitner 2015b). All studies reported on clinical pregnancy, but if they did not report on live birth, we classified them at unclear risk of bias (Cambiaghi 2013; Dehghani Firouzabadi 2016; Eskandar 2016; Hong 2014; Hosseini 2016; Huang 2016; Kokkali 2014; Leao 2013; Mostajeran 2017; Navali 2016; Santibañez 2014; Zarei 2014).

\section{Other potential sources of bias}

We classified 12 studies at low risk of other potential bias because groups appeared to be comparable at baseline, and we could not identify any other sources of bias (Aaleyasin 2015; Dehghani Firouzabadi 2016; Eskandar 2016; Hosseini 2016; Huang 2016; Mostajeran 2017; Navali 2016; Santibañez 2014; Singh 2014; Wirleitner 2015a; Wirleitner 2015b; Zarei 2014). We classified three studies at unclear risk of bias because they did not report on baseline characteristics between groups (probably because they were available in abstract format only) (Cambiaghi 2013; Kokkali 2014), or they reported a large number of participants who declined to participate after randomisation for various reasons (Hong 2014). We classified two studies at high risk of bias owing to lack of reporting of participant numbers in each study group in Leao 2013, and owing to performance of an interim analysis that changed the study protocol and ended the study prematurely in Mansour 2011.

The overall birth rate in the control groups in Mansour 2011 was $47 \%$, whereas the control group live birth rate ranged from $25 \%$ to $39 \%$ in the other included studies. The reason for this was unclear. The mean age of women in Mansour 2011 was under 30 years, but this was also the case in Aaleyasin 2015, which reported a control group live birth rate of only $25 \%$. Furthermore, Mansour 2011 randomised women at the beginning of their cycle, and Aaleyasin 2015 randomised women before embryo transfer, which should have led to higher live birth rates (by not including cancelled cycles).

\section{Assessment of publication bias}

The funnel plot for clinical pregnancy did not show any evidence of publication bias (Figure 4). 
Figure 4. Funnel plot of comparison: 1 Intrauterine human chorionic gonadotropin (hCG) versus no hCG, outcome: 1.4 Clinical pregnancy.

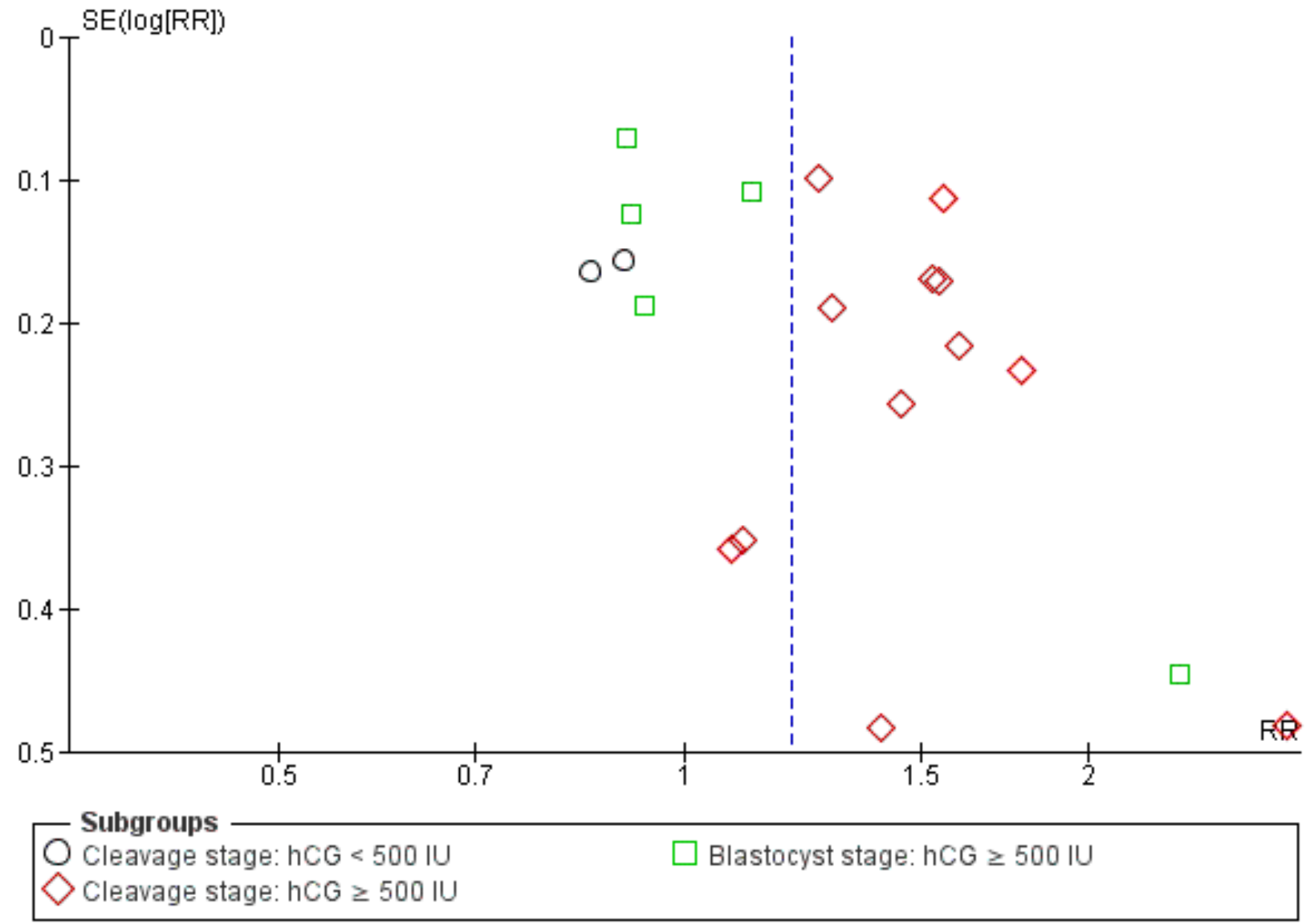

\section{Effects of interventions}

See: Summary of findings for the main comparison Intrauterine administration of hCG for women undergoing assisted reproduction

Note: One study included three experimental arms (Mansour 2011), and another study included two experimental arms based on intrauterine hCG dose (i.e. $100 \mathrm{IU}, 200 \mathrm{IU}, 500 \mathrm{IU}$, and 1000 IU, respectively) (Dehghani Firouzabadi 2016). We regarded and analysed them as separate comparisons. We split the control groups proportionately with the experimental groups to avoid analysing control participants in duplicate. One study investigated intrauterine hCG administration at two different timings (day three vs day five administration), and we regarded and analysed them as two separate comparisons (Wirleitner 2015a).

Two of the comparisons showed considerable heterogeneity $\left(\mathrm{I}^{2}>\right.$ $75 \%$ ) (Analysis 1.1; Analysis 1.4), and we did not perform a global meta-analysis as prespecified in the protocol (Craciunas 2015).

Exploration for the sources of heterogeneity in these analyses revealed two key prespecified variables as important determinants: stage of ET (cleavage vs blastocyst stage) and dose of IC-hCG (<500 IU vs $\geq 500 \mathrm{IU}$ ). When we subgrouped the data according to these variables, we found evidence of significant differences between subgroups. We then performed meta-analysis within the subgroups defined by stage of embryo and dose of hCG.

\section{Primary outcomes}

\section{Live birth}

(Analysis 1.1)

Five studies with eight experimental arms reported on live birth (Aaleyasin 2015; Mansour 2011; Singh 2014; Wirleitner 2015a; Wirleitner 2015b) (Analysis 1.1).

\section{Subgroup analysis}

The forest plot displayed these studies based on the embryo stage at transfer and the hCG dose (Figure 5). The test for subgroup differences indicated a considerable difference between subgroups $\left(\mathrm{Chi}^{2}=29.39\right.$, degrees of freedom $\left.(\mathrm{df})=2, \mathrm{P} \leq 0.00001, \mathrm{I}^{2}=92.3 \%\right)$. 
Figure 5. Forest plot of comparison: 1 Intrauterine human chorionic gonadotropin (hCG) versus no hCG, outcome: 1.1 Live birth.

\begin{tabular}{|c|c|c|c|c|c|c|c|c|c|}
\hline \multirow{2}{*}{ Study or Subgroup } & \multicolumn{2}{|c|}{ Intrauterine hCG } & \multicolumn{2}{|c|}{ No hCG } & \multirow[b]{2}{*}{ Weight } & \multirow{2}{*}{$\begin{array}{c}\text { Risk Ratio } \\
\text { M-H, Random, } 95 \% \mathrm{CI}\end{array}$} & \multirow{2}{*}{\multicolumn{2}{|c|}{$\begin{array}{c}\text { Risk Ratio } \\
\text { M-H, Random, } 95 \% \mathrm{Cl}\end{array}$}} & Risk of Bias \\
\hline & Events & Total & Events & Total & & & & & A B C D E F G \\
\hline \multicolumn{10}{|c|}{ 1.1.1 Cleavage stage: $\mathrm{hCG}<500 \mathrm{IU}$} \\
\hline Mansour 2011 (1) & 35 & 92 & 23 & 47 & $49.2 \%$ & $0.78[0.53,1.15]$ & & - & ๑? \\
\hline Mansour $2011(2)$ & 35 & 93 & 24 & 48 & $50.8 \%$ & $0.75[0.51,1.11]$ & & & \\
\hline Subtotal $(95 \% \mathrm{Cl})$ & & 185 & & 95 & $100.0 \%$ & $0.76[0.58,1.01]$ & & & \\
\hline Total events & 70 & & 47 & & & & & & \\
\hline \multirow{2}{*}{\multicolumn{10}{|c|}{$\begin{array}{l}\text { Heterogeneity: } \operatorname{Tau}^{2}=0.00 ; \mathrm{Chi}^{2}=0.01, \mathrm{df}=1(\mathrm{P}=0.91) ; \mathrm{I}^{2}=0 \% \\
\text { Test for overall effect: } \mathrm{Z}=1.91(\mathrm{P}=0.06)\end{array}$}} \\
\hline & & & & & & & & & \\
\hline \multicolumn{10}{|c|}{ 1.1.2 Cleavage stage: $\mathrm{hCG} \geq 500 \mathrm{IU}$} \\
\hline Aaleyasin 2015 & 98 & 240 & 60 & 243 & $43.6 \%$ & $1.65[1.27,2.16]$ & & & 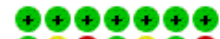 \\
\hline Mansour 2011 (3) & 66 & 108 & 45 & 107 & $43.1 \%$ & $1.45[1.11,1.90]$ & & $\longrightarrow$ & \\
\hline Singh 2014 & 34 & 108 & 20 & 108 & $13.3 \%$ & $1.70[1.05,2.76]$ & & & \\
\hline Subtotal $(95 \% \mathrm{Cl})$ & & 456 & & 458 & $100.0 \%$ & $1.57[1.32,1.87]$ & & & \\
\hline Total events & 198 & & 125 & & & & & & \\
\hline \multirow{2}{*}{\multicolumn{10}{|c|}{$\begin{array}{l}\text { Heterogeneity: } \operatorname{Tau}^{2}=0.00 ; \mathrm{Chi}^{2}=0.59, \mathrm{df}=2(\mathrm{P}=0.75) ; \mathrm{I}^{2}=0 \% \\
\text { Test for overall effect: } Z=5.01(\mathrm{P}=0.00001)\end{array}$}} \\
\hline & & & & & & & & & \\
\hline \multicolumn{10}{|c|}{ 1.1.3 Blastocyst stage: hCG $\geq 500 \mathrm{IU}$} \\
\hline Wirleitner 2015a (4) & 31 & 89 & 34 & 93 & $11.0 \%$ & $0.95[0.64,1.41]$ & & & ๑) ?૯?૯૯ \\
\hline Wirleitner 2015a (5) & 188 & 510 & 198 & 494 & $68.3 \%$ & $0.92[0.79,1.08]$ & & - & ๑? ?૯?૯૯ \\
\hline Wirleitner 2015b & 68 & 255 & 68 & 225 & $20.7 \%$ & $0.88[0.66,1.17]$ & & - & ? ? $\oplus \oplus \oplus \oplus \oplus$ \\
\hline Subtotal $(95 \% \mathrm{Cl})$ & & 854 & & 812 & $100.0 \%$ & $0.92[0.80,1.04]$ & & & \\
\hline Total events & 287 & & 300 & & & & & & \\
\hline \multirow{2}{*}{\multicolumn{10}{|c|}{$\begin{array}{l}\text { Heterogeneity: } \operatorname{Tau}^{2}=0.00 ; \mathrm{Chi}^{2}=0.11, \mathrm{df}=2(\mathrm{P}=0.95) ; \mathrm{I}^{2}=0 \% \\
\text { Test for overall effect: } Z=1.34(P=0.18)\end{array}$}} \\
\hline & & & & & & & & & \\
\hline & & & & & & & 0.5 & 1.5 & \\
\hline \multirow{2}{*}{\multicolumn{10}{|c|}{ Test for subgroup differences: $\mathrm{Chi}^{2}=29.39, \mathrm{df}=2(\mathrm{P}<0.00001), \mathrm{I}^{2}=93.2 \%$}} \\
\hline Footnotes & rences. & & & & & & & & \\
\hline (1) hCG $100 \mathrm{IU}$ & & & & & & & (A) Random sequenc & e generation & bias) \\
\hline (2) hCG $200 \mathrm{IU}$ & & & & & & & (B) Allocation conceal & ment (select & \\
\hline (3) hCG 500 IU & & & & & & & (C) Blinding of particip & ants and pe & Iformance bias) \\
\hline (4) Day 3 hCG admir & tration & & & & & & (D) Blinding of outcom & he assessm & on bias) \\
\hline \multirow{3}{*}{\multicolumn{2}{|c|}{ (5) Day 5 hCG administration }} & & & & & & (E) Incomplete outcon & he data (attrit & \\
\hline & & & & & & & (F) Selective reporting & (reporting b & \\
\hline & & & & & & & (G) Other bias & & \\
\hline
\end{tabular}

- Cleavage stage: IC-hCG less than 500 IU versus no IC-hCG: one RCT with two experimental arms contributed to calculation of the combined outcome (Mansour 2011). The heterogeneity was insignificant $\left(\mathrm{Chi}^{2}=0.01, \mathrm{df}=1, \mathrm{P}=0.91, \mathrm{I}^{2}=0 \%\right)$, and findings showed no benefit of the intervention, which was consistent with no substantive difference or disadvantage of indeterminate magnitude (RR $0.76,95 \% \mathrm{Cl} 0.58$ to 1.01 ; one RCT; $\mathrm{N}=280 ; \mathrm{I}^{2}=$ $0 \%$; very low-quality evidence).

- Cleavage stage: IC-hCG 500 IU or greater versus no IC-hCG: three RCTs contributed to calculation of the combined outcome (Aaleyasin 2015; Mansour 2011; Singh 2014). The heterogeneity was insignificant $\left(\mathrm{Chi}^{2}=0.59, \mathrm{df}=2, \mathrm{P}=0.75, \mathrm{I}^{2}=0 \%\right)$, and the live birth rate was higher in the hCG group (RR 1.57, 95\% Cl 1.32 to 1.87 ; three RCTs; $N=914 ; I^{2}=0 \%$; moderate-quality evidence). This suggested that in women with a $27 \%$ chance of live birth without using IC-hCG, the live birth rate among those using IChCG 500 IU or greater will be between $36 \%$ and $51 \%$.

- Blastocyst stage: IC-hCG 500 IU or greater versus no IC-hCG: two RCTs with three experimental arms contributed to calculation of the combined outcome (Wirleitner 2015a; Wirleitner 2015b). The heterogeneity was insignificant $\left(\mathrm{Chi}^{2}=0.11, \mathrm{df}=2, \mathrm{P}=0.95, \mathrm{I}^{2}\right.$ $=0 \%$ ), and results showed no substantive differences between groups in live birth rates (RR 0.92, 95\% Cl 0.80 to 1.04; two RCTs; $\mathrm{N}=1666 ; \mathrm{I}^{2}=0 \%$; moderate-quality evidence).
Data were insufficient for the prespecified subgroup analyses to be performed based on embryo processing and number of embryos transferred.

\section{Sensitivity analyses}

Removing studies with high risk of bias in one or more domains did not alter the results significantly (Mansour 2011; Singh 2014; Wirleitner 2015a), but it meant that no data were available for one of the comparisons.

- Cleavage stage: IC-hCG less than 500 IU versus no IC-hCG (no data).

- Cleavage stage: IC-hCG 500 IU or greater versus no IC-hCG (RR $1.65,95 \% \mathrm{Cl} 1.27$ to 2.16 ; one RCT; $\mathrm{N}=483$ ).

- Blastocyst stage: IC-hCG 500 IU or greater versus no IC-hCG (RR $0.88,95 \% \mathrm{Cl} 0.66$ to 1.17 ; one RCT; $\mathrm{N}=480$ ).

Removing the studies available in abstract format only did not alter the results significantly (Singh 2014; Wirleitner 2015b).

- Cleavage stage: IC-hCG less than 500 IU versus no IC-hCG (RR $0.76,95 \% \mathrm{Cl} 0.58$ to 1.01 ; one $\mathrm{RCT} ; \mathrm{N}=280 ; \mathrm{I}^{2}=0 \%$; very lowquality evidence).

- Cleavage stage: IC-hCG 500 IU or greater versus no IC-hCG (RR $1.55,95 \% \mathrm{Cl} 1.28$ to 1.87 ; two RCTs; $\mathrm{N}=698 ; \mathrm{I}^{2}=0 \%$; moderatequality evidence). 
- Blastocyst stage: IC-hCG 500 IU or greater versus no IC-hCG (RR $0.92,95 \% \mathrm{Cl} 0.80$ to 1.07 ; one RCT; $\mathrm{N}=1186 ; \mathrm{I}^{2}=0 \%$; moderatequality evidence).

The calculated combined outcome based on the fixed-effect model was similar to that based on the random-effects model for the following.

- Cleavage stage: IC-hCG less than $500 \mathrm{IU}$ versus no IC-hCG (RR $0.76,95 \% \mathrm{Cl} 0.58$ to 1.01 ; one $\mathrm{RCT} ; \mathrm{N}=280 ; \mathrm{I}^{2}=0 \%$; very lowquality evidence).

- Cleavage stage: IC-hCG 500 IU or greater versus no IC-hCG (RR $1.59,95 \% \mathrm{Cl} 1.33$ to 1.90 ; three RCTs; $\mathrm{N}=914 ; \mathrm{I}^{2}=0 \%$; moderatequality evidence).

- Blastocyst stage: IC-hCG 500 IU or greater versus no IC-hCG (RR $0.91,95 \% \mathrm{Cl} 0.80$ to 1.04 ; two RCTs; $\mathrm{N}=1666 ; \mathrm{I}^{2}=0 \%$; moderatequality evidence).

Results did not differ substantially when odds ratio (OR) was used instead of risk ratio (RR).
- Cleavage stage: IC-hCG less than 500 IU versus no IC-hCG (OR $0.62,95 \% \mathrm{Cl} 0.38$ to 1.03 ; one $\mathrm{RCT} ; \mathrm{N}=280 ; \mathrm{I}^{2}=0 \%$; very lowquality evidence).

- Cleavage stage: IC-hCG 500 IU or greater versus no IC-hCG (OR $2.10,95 \% \mathrm{Cl} 1.59$ to 2.79 ; three RCTs; $\mathrm{N}=914 ; \mathrm{I}^{2}=0 \%$; moderatequality evidence).

- Blastocyst stage: IC-hCG 500 IU or greater versus no IC-hCG (OR $0.87,95 \% \mathrm{Cl} 0.71$ to 1.06 ; two RCTs; $\mathrm{N}=1666 ; \mathrm{I}^{2}=0 \%$; moderatequality evidence).

\section{Miscarriage}

(Analysis 1.2)

Eleven studies with 15 experimental arms reported on miscarriage (Aaleyasin 2015; Dehghani Firouzabadi 2016; Hong 2014; Hosseini 2016; Huang 2016; Mansour 2011; Navali 2016; Singh 2014; Wirleitner 2015a; Wirleitner 2015b; Zarei 2014; Analysis 1.2; Figure 6). Heterogeneity between studies was unsubstantial $\left(\mathrm{Chi}^{2}=6.95\right.$, $\left.\mathrm{df}=14, \mathrm{P}=0.74, \mathrm{I}^{2}=0 \%\right)$, and studies provided no evidence of a difference between groups in miscarriage rates (RR 1.04, 95\% Cl 0.81 to $1.35 ; 11$ RCTs; $N=3927 ;\left.\right|^{2}=0 \%$; very low-quality evidence).

Figure 6. Forest plot of comparison: 1 Intrauterine human chorionic gonadotropin (hCG) versus no hCG, outcome: 1.2 Miscarriage.

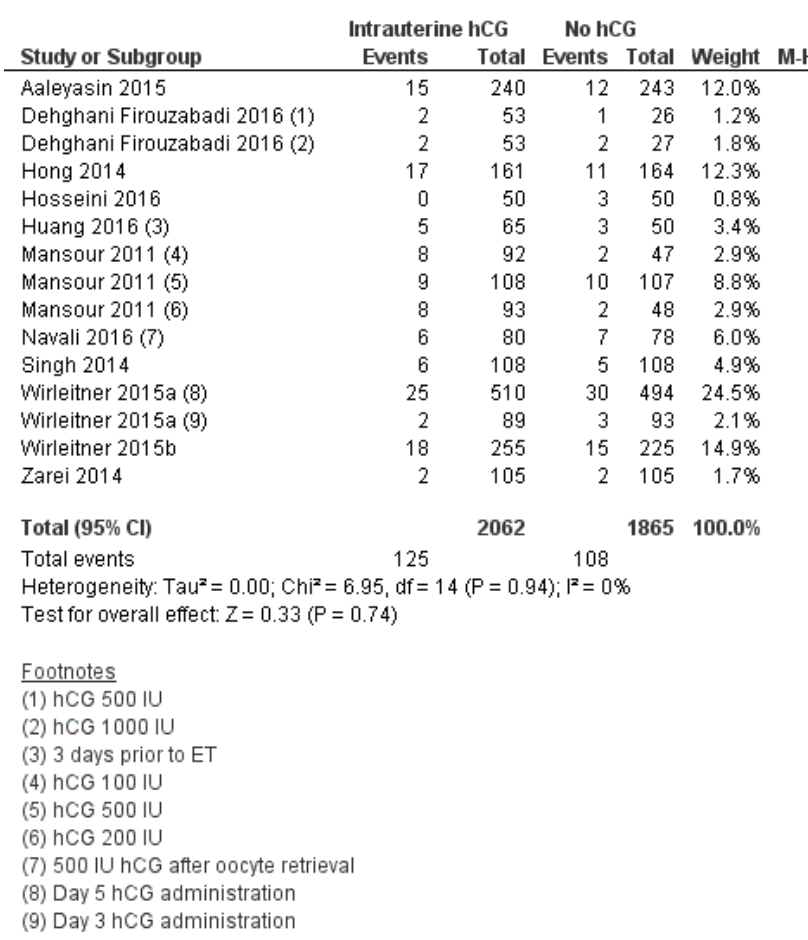

Risk Ratio Risk Ratio

Random, $95 \% \mathrm{Cl}$

$1.27[0.61,2.65]$

$0.98[0.09,10.33]$

$0.51[0.08,3.42]$

$1.57[0.76,3.26]$

$0.14[0.01,2.70]$

$1.28[0.32,5.11]$

$2.04[0.45,9.24]$

$0.89[0.38,2.11]$

$2.06[0.46,9.34]$

$0.84[0.29,2.38]$

$1.20[0.38,3.81]$

$0.81[0.48,1.35]$

$0.70[0.12,4.07]$

$1.06[0.55,2.05]$

$1.00[0.14,6.97]$

$1.04[0.81,1.35]$

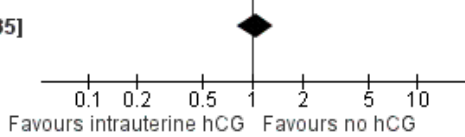

\section{Risk of bias legend}

(A) Random sequence generation (selection bias)

(B) Allocation concealment (selection bias)

(C) Blinding of participants and personnel (performance bias)

(D) Blinding of outcome assessment (detection bias)

(E) Incomplete outcome data (attrition bias)

(F) Selective reporting (reporting bias)

(G) Other bias

\section{Sensitivity analyses}

Removing studies with high risk of bias in one or more domains Dehghani Firouzabadi 2016, Hosseini 2016, Mansour 2011, Navali 2016, Singh 2014, and Wirleitner 2015a - did not alter the results significantly (RR $1.26,95 \% \mathrm{Cl} 0.86$ to 1.84 ; five RCTs; $\mathrm{N}=1613 ; \mathrm{I}^{2}=$ $0 \%$; very low-quality evidence).

Removing the two studies available in abstract format only - Singh 2014 and Wirleitner 2015b - did not alter the results significantly (RR
$1.03,95 \% \mathrm{Cl} 0.78$ to 1.37 ; nine RCTs; $\mathrm{N}=3231 ;\left.\right|^{2}=0 \%$; very lowquality evidence).

The calculated combined outcome based on the fixed-effect model was similar to that based on the random-effects model (RR 1.04, $95 \% \mathrm{Cl} 0.81$ to $1.34 ; 11 \mathrm{RCTs}$; $=3927 ; I^{2}=0 \%$; very low-quality evidence). 
Results did not differ substantially when OR was used instead of RR (OR $1.05,95 \% \mathrm{Cl} 0.80$ to $1.37 ; 11 \mathrm{RCTs} ; \mathrm{N}=3927 ; \mathrm{I}^{2}=0 \%$; very lowquality evidence).

\section{Secondary analysis per clinical pregnancy}

\section{(Analysis 1.3)}

Studies provided no evidence of a difference between groups in miscarriage rates calculated per clinical pregnancy (RR $0.84,95 \% \mathrm{Cl}$ 0.62 to $1.13 ; 11$ RCTs; $N=1620 ; I^{2}=24 \%$; very low-quality evidence) (Analysis 1.3).

\section{Secondary outcomes}

\section{Clinical pregnancy}

(Analysis 1.4)

All included studies reported clinical pregnancy (Analysis 1.4).

\section{Subgroup analysis}

The forest plot displayed the studies based on embryo stage at transfer and hCG dose (Figure 7). The test for subgroup differences indicated a considerable difference between subgroups $\left(\mathrm{Chi}^{2}=\right.$ $\left.25.95, \mathrm{df}=2, \mathrm{P} \leq 0.00001, \mathrm{I}^{2}=92.3 \%\right)$.

Figure 7. Forest plot of comparison: 1 Intrauterine human chorionic gonadotropin (hCG) versus no hCG, outcome: 1.4 Clinical pregnancy.

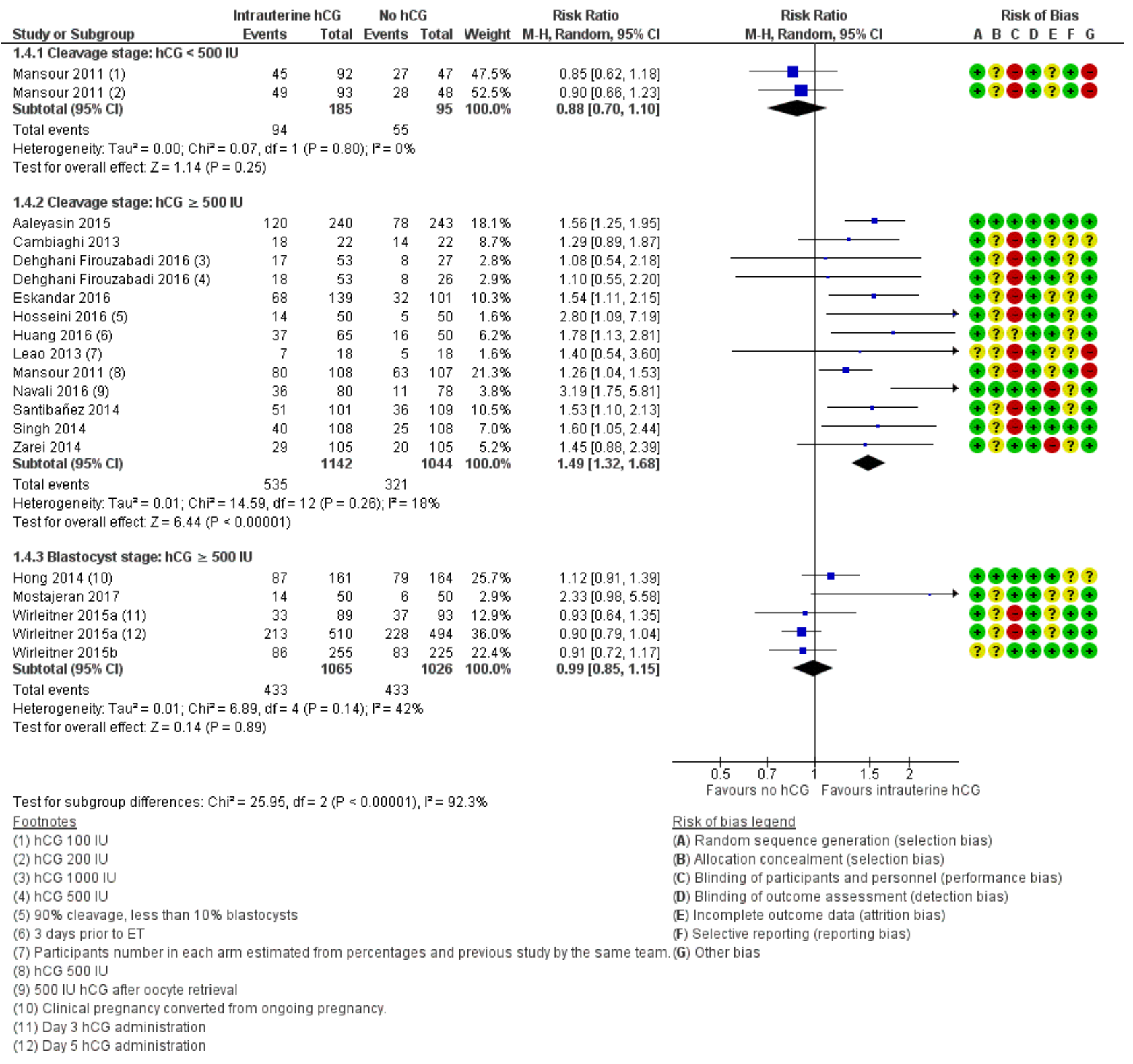

- Cleavage stage: IC-hCG less than 500 IU versus no IC-hCG: one RCT with two experimental arms contributed to calculation of the combined outcome (Mansour 2011). Heterogeneity was insignificant $\left(\mathrm{Chi}^{2}=0.07, \mathrm{df}=1, \mathrm{P}=0.80, \mathrm{I}^{2}=0 \%\right)$, and studies 
provided no evidence of a difference between groups in clinical pregnancy rates (RR $0.88,95 \% \mathrm{Cl} 0.70$ to 1.10 ; one $\mathrm{RCT}$; $\mathrm{N}=280$; $\mathrm{I}^{2}=0 \%$; very low-quality evidence).

- Cleavage stage: IC-hCG 500 IU or greater versus no IC-hCG: 12 RCTs contributed to calculation of the combined outcome (Aaleyasin 2015; Cambiaghi 2013; Dehghani Firouzabadi 2016; Eskandar 2016; Hosseini 2016; Huang 2016; Leao 2013; Mansour 2011; Navali 2016; Santibañez 2014; Singh 2014; Zarei 2014). Heterogeneity was insignificant $\left(\mathrm{Chi}^{2}=14.59, \mathrm{df}=12, \mathrm{P}=0.26\right.$, $\left.1^{2}=18 \%\right)$, and the clinical pregnancy rate was higher in the hCG group (RR $1.49,95 \% \mathrm{Cl} 1.32$ to $1.68 ; 12$ RCTs; $\mathrm{N}=2186$; $\mathrm{I}^{2}=18 \%$; moderate-quality evidence).

One study investigated IC-hCG $500 \mathrm{IU}$ and reported no evidence of a difference between groups in clinical pregnancy rates (Kokkali 2014). Data from this study were insufficient to be included in the meta-analysis.

- Blastocyst stage: IC-hCG 500 IU or greater versus no IC-hCG: four RCTs with five experimental arms contributed to calculation of the combined outcome (Hong 2014; Mostajeran 2017; Wirleitner 2015a; Wirleitner 2015b). Heterogeneity was moderate $\left(\mathrm{Chi}^{2}=\right.$ $6.89, \mathrm{df}=4, \mathrm{P}=0.14, \mathrm{I}^{2}=42 \%$ ), and studies provided no evidence of a difference between groups in clinical pregnancy rates (RR
$0.99,95 \% \mathrm{Cl} 0.85$ to 1.15 ; four RCTs; $\mathrm{N}=2091 ;\left.\right|^{2}=42 \%$; moderatequality evidence).

Data were insufficient for the predefined subgroup analyses to be performed based on embryo processing and number of embryos transferred.

\section{Complications}

(Analysis 1.5)

Seven studies with 10 experimental arms reported complications (Aaleyasin 2015; Dehghani Firouzabadi 2016; Hosseini 2016; Mansour 2011; Navali 2016; Santibañez 2014; Zarei 2014; Analysis 1.5).

Evidence was insufficient to show whether there was a difference between groups for any of the mentioned complications: ectopic pregnancy (four studies; $\mathrm{N}=1073$; four events overall), heterotopic pregnancy (one study; $\mathrm{N}=495$; one event), intrauterine death (three studies; $N=1078 ; 22$ events), and triplets (one study; $N=48$; three events). For intrauterine death, the analysis in Figure 8 displays the Peto OR (which is the default setting for this analysis). MantelHaenszel random-effects RRs were almost identical (RR 0.77, 95\% $\mathrm{Cl} 0.33$ to 1.77 ; three studies; $\left.\mathrm{N}=1078 ; \mathrm{I}^{2}=0 \%\right)$. 
Figure 8. Forest plot of comparison: 1 Intrauterine human chorionic gonadotropin (hCG) versus no hCG, outcome: 1.5 Complications.

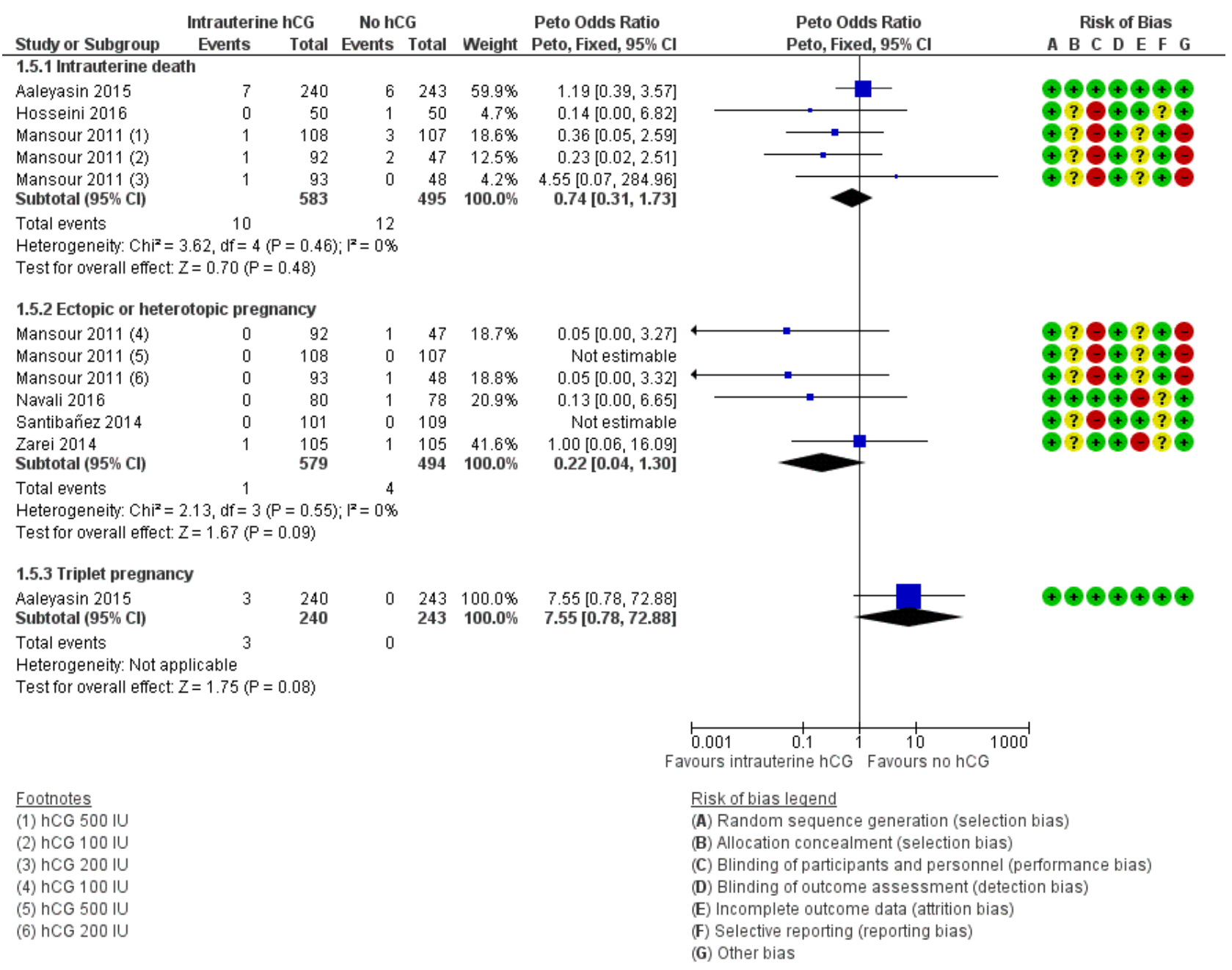

\section{DISCUSSION}

\section{Summary of main results}

This updated systematic review included 17 randomised controlled trials (RCTs) investigating the effect of intrauterine administration of human chorionic gonadotropin (hCG) to 4751 subfertile women undergoing assisted reproduction. Intracavitary hCG (IC-hCG) was administered in variable doses at different times before embryo transfer (ET). hCG was obtained from the urine of pregnant women or from cell cultures using recombinant DNA technology.

For analyses of live birth and clinical pregnancy, there was considerable heterogeneity $\left(1^{2}>75 \%\right)$ and therefore we present subgroups for dosage and stage of ET. Exploration for the sources of heterogeneity revealed two key prespecified variables as important determinants: stage of ET (cleavage vs blastocyst stage) and dose of IC-hCG (<500 IU vs $\geq 500 \mathrm{IU})$. We performed meta-analysis within the subgroups defined by stage of embryo and dose of IC-hCG.

Live birth rates among women having cleavage-stage ET with an IChCG dose < 500 IU compared to women having cleavage-stage ET without IC-hCG showed no benefit of the intervention and would be consistent with no substantive difference or disadvantage of indeterminate magnitude. In a clinic with a live birth rate of $49 \%$ per cycle, use of IC-hCG < $500 \mathrm{IU}$ would be associated with a live birth rate ranging from $28 \%$ to $50 \%$.

Results show an increase in live birth rate in the subgroup of women undergoing cleavage-stage ET with an IC-hCG dose $\geq 500$ IU compared to women having cleavage-stage ET without IC-hCG (RR 1.57, 95\% Cl 1.32 to 1.87; three RCTs; 914 participants; I $^{2}=0 \%$; moderate-quality evidence). At a clinic with a live birth rate of $27 \%$ per cycle, use of IC-hCG $\geq 500$ IU would be associated with a live birth rate ranging from $36 \%$ to $51 \%$.

Results show no substantive differences in live birth among women having blastocyst-stage ET with an IC-hCG dose $\geq 500$ IU compared to women having blastocyst-stage ET without IC-hCG (moderatequality evidence). At a clinic with a live birth rate of $36 \%$ per cycle, use of IC-hCG $\geq 500 \mathrm{IU}$ would be associated with a live birth rate ranging from $29 \%$ to $38 \%$.

Evidence for clinical pregnancy among women having cleavagestage ET with an IC-hCG dose < 500 IU showed no benefit of the intervention and would be consistent with no substantive 
difference or disadvantage of indeterminate magnitude (very lowquality evidence).

Results show an increase in clinical pregnancy rate in the subgroup of women having cleavage-stage ET with an IC-hCG dose of 500 IU or greater compared to women having cleavage-stage ET with no IC-hCG (moderate-quality evidence).

Results show no substantive differences in clinical pregnancy in the subgroup of women having blastocyst-stage ET with an IC-hCG dose of 500 IU or greater (moderate-quality evidence).

No RCTs investigated blastocyst-stage ET with an IC-hCG dose $<500$ IU.

We are uncertain whether miscarriage and complication rates were influenced by IC-hCG administration, irrespective of embryo stage at transfer or dose of IC-hCG (very low-quality evidence). Reported complications were few, and very low-quality evidence was insufficient to permit conclusions to be drawn.

\section{Overall completeness and applicability of evidence}

All RCTs reported on clinical pregnancy, which is an important secondary outcome, but only a few RCTs continued follow-up until live birth, which is the most important primary outcome.

Most RCTs reported miscarriage rates. RCTs rarely reported complications and adverse events, or their absence.

Data were insufficient for all planned subgroup analyses to be performed.

The inclusion criteria for participants ensured a broad range of subfertility causes and women's characteristics similar to those expected in a regular assisted reproduction unit.

\section{Quality of the evidence}

We rated most of the studies $(12 / 17)$ at high risk of bias in at least one of the seven domains assessed. Common problems were unclear reporting of study methods and lack of blinding. Brief reporting of results in studies published as abstracts represents an additional potential source of bias. Ten studies did not report funding, and seven studies reported internal funding. No studies reported external funding.

The quality of the evidence as assessed via GRADE varied from very low to moderate for live birth and clinical pregnancy, which means that further research is likely to have an important impact on our confidence in the estimate of effect and may change the estimate for some subgroups. The quality of the evidence for miscarriage was very low, meaning that we are very uncertain about the estimate. The main limitations in the overall quality of the evidence were high risk of bias and serious imprecision.

\section{Potential biases in the review process}

We performed a systematic search in consultation with the Cochrane Gynaecology and Fertility Group Trials Search Coordinator, but we cannot be sure all relevant trials were identified for inclusion. The protocol was pre-published and was followed accordingly (Craciunas 2015). We attempted to contact study authors when data were missing, but only one study author replied, providing clarification and additional data (Mansour 2011). We performed analyses on an intention-to-treat basis. Potential bias in the review process was unlikely. Data from two studies awaiting classification and from five ongoing studies may inform future updates of this review.

\section{Agreements and disagreements with other studies or reviews}

One previously published meta-analysis concluded that women undergoing in vitro fertilisation (IVF)/intracytoplasmic sperm injection (ICSI) may benefit from IC-hCG injection before ET (Ye 2015). One meta-analysis found no effect of IC-hCG in terms of live birth and miscarriage but reported increased clinical pregnancy following IC-hCG injection (Osman 2016). A third meta-analysis published as an abstract reported increased clinical pregnancy rates and similar implantation, miscarriage, and ongoing pregnancy rates following IC-hCG (Dieamant 2016). These previous meta-analyses included significantly fewer RCTs compared to the present review (five, eight, and six, respectively) and have not explored the sources of heterogeneity based on IChCG dose and embryo stage at transfer.

The reported effect of intrauterine hCG administration was consistent within the subgroups of our review, with an apparent different effect based on stage of the embryo at transfer and dose of IC-hCG.

\section{AUTHORS' CONCLUSIONS}

\section{Implications for practice}

The finding of probably improved clinical pregnancy and live birth for cleavage-stage transfers using an intracavity human chorionic gonadotropin (IC-hCG) dose of 500 IU or greater is clinically important. Given the strength of the evidence, we believe that patients will benefit from this intervention, and it could be incorporated into clinical practice. However, current evidence for IC-hCG treatment does not support its use for blastocyst transfers. Review authors found no evidence that miscarriage was influenced by intrauterine human chorionic gonadotropin (hCG) administration, irrespective of embryo stage at transfer or dose of IC-hCG. Events were too few to allow any conclusions to be drawn with regard to other complications.

\section{Implications for research}

The findings of this review should provide a strong foundation for funding and conducting further high-quality randomised controlled trials of intrauterine hCG administration for women undergoing assisted reproduction according to CONSORT (Consolidated Standards of Reporting Trials) guidelines. These trials should be powered adequately and should focus on subgroups (cleavage vs blastocyst, fresh vs frozen/thawed, single vs two or more embryo transfers, cause of subfertility, dose and timing of IC-hCG) to identify the groups of women who would benefit the most from this intervention, and they should report on potential adverse events. Live birth rate must be the primary outcome. Blinding throughout the treatment cycle and during embryo transfer may reduce potential performance bias (adjusting ovarian stimulation doses; deciding the timing of maturation triggering, oocyte retrieval, and technique during embryo transfer, respectively). 


\section{ACK N OWLEDGEMENTS}

We thank Helen Nagels (Managing Editor), Marian Showell (Information Specialist), and the editorial board of the Cochrane
Gynaecology and Fertility Group for their invaluable assistance in developing this review.

We thank our peer reviewers for providing helpful and constructive feedback. 


\section{R E F E R E N C E S}

\section{References to studies included in this review}

\section{Aaleyasin 2015 \{published data only\}}

Aaleyasin A, Aghahosseini M, Rashidi M, Safdarian L, Sarvi F, Najmi Z, et al. In vitro fertilization outcome following embryo transfer with or without preinstillation of human chorionic gonadotropin into the uterine cavity: a randomized controlled trial. Gynecologic and Obstetric Investigation 2015;79(3):201-5. [DOI: 10.1159/000363235; PUBMED: 25531413]

\section{Cambiaghi 2013 \{published data only\}}

Cambiaghi AS, Leao RBF, Alvarez AV, Nascimento PF. Intrauterine injection of human chorionic gonadotropin before embryo transfer may improve clinical pregnancy and implantation rates in blastocysts transfers. Fertility and Sterility 2013;100(3):S121. [DOI: 10.1016/j.fertnstert.2013.07.1634]

\section{Dehghani Firouzabadi 2016 \{published data only\}}

Dehghani Firouzabadi R, Janati, Razi MH. The effect of intrauterine human chorionic gonadotropin injection before embryo transfer on the implantation and pregnancy rate in infertile patients: a randomized clinical trial. International Journal of Reproductive Biomedicine 2016;14(10):657-64. [PUBMED: 27921090]

Janati S, Dehghani Firouzabadi R, Mohseni F, Razi MH. Evaluation effect of intrauterine human chorionic gonadotropin injection before embryo transfer in implantation and pregnancy rate in infertile patients and comparison with conventional embryo transfer in IVF/ICSI/ET cycles. Iranian Journal of Reproductive Medicine 2013;11(4):67-8.

\section{Eskandar 2016 \{published and unpublished data\}}

Eskandar MA, Al-Emain MA, Atwan YL, Bakar SD. Does intrauterine injection of human chorionic gonadotropin before embryo transfer improve the pregnancy rate in in vitro fertilization/intracytoplasmic sperm injection (IVFICSI) cycles? A prospective randomized controlled trial. Reproductive Sciences 2016;23(1 Suppl):102.

\section{Hong 2014 \{published data only\}}

Hong KH, Forman EJ, Werner MD, Upham KM, Gumeny CL, Winslow $A D$, et al. Endometrial infusion of human chorionic gonadotropin at the time of blastocyst embryo transfer does not impact clinical outcomes: a randomized, doubleblind, placebo-controlled trial. Fertility and Sterility 2014;102(6):1591-5. [DOI: 10.1016/j.fertnstert.2014.08.006; PUBMED: 25234040]

\section{Hosseini 2016 \{published data only\}}

Hosseini RS, Farzadi L, Abdollahi S, Nouri M, Ghasemzadeh A, Hamdi K, et al. Effect of intrauterine injection of human chorionic gonadotropin before frozen-thawed embryo transfer on implantation and clinical pregnancy rate: a randomized controlled trial. International Journal of Women's Health and Reproduction Sciences 2016;4(4):189-93. [DOI: 10.15296/ ijwhr.2016.42]
Huang 2016 \{published data only\}

Huang P, Wei L, Li X. A study of intrauterine infusion of human chorionic gonadotropin (hCG) before frozenthawed embryo transfer after two or more implantation failures. Gynecological Endocrinology 2017;33(1):67-9. [DOI: 10.1080/09513590.2016.1207164; PUBMED: 27449969]

\section{Kokkali 2014 \{published data only\}}

Kokkali G, Chronopoulou M, Baxevani E, Biba M, Aggeli I, Fakiridou M, et al. A randomised control pilot study of the use of intrauterine human chorionic gonadotropin injection before embryo transfer in egg recipient cycles. Human Reproduction 2014;29(Suppl 1):i208.

\section{Leao 2013 \{published data only\}}

Leao RBF, Cambiaghi AS, Leao BF, Alvarez ABV, Figueiredo PN. Intrauterine injection of human chorionic gonadotropin before embryo transfer may improve the pregnancy rates in in vitro fertilization cycles of patients with repeated implantation failures. Proceedings of the 5th IVI International Congress; 2013 Apr 4-6; Seville, Spain. http://www.comtecmed.com/ivi/2013/ Uploads/Editor/abstract_66.pdf. 2013. [http://comtecmed.com/ ivi/2013/Uploads/Editor/abstract_66.pdf]

\section{Mansour 2011 \{published and unpublished data\}}

Mansour R. Re: Intrauterine hCG [personal communication]. Email to: L Craciunas 10 June 2015.

* Mansour R, Tawab N, Kamal O, El-Faissal Y, Serour A, Aboulghar M, et al. Intrauterine injection of human chorionic gonadotropin before embryo transfer significantly improves the implantation and pregnancy rates in in vitro fertilization/ intracytoplasmic sperm injection: a prospective randomized study. Fertility and Sterility 2011;96(6):1370-4. [DOI: 10.1016/ j.fertnstert.2011.09.044; PUBMED: 22047664]

\section{Mostajeran 2017 \{published data only\}}

Mostajeran F, Godazandeh F, Ahmadi SM, Movahedi M, Jabalamelian SA. Effect of intrauterine injection of human chorionic gonadotropin before embryo transfer on pregnancy rate: a prospective randomized study. Journal of Research in Medical Sciences 2017;22:6. [DOI: 10.4103/1735-1995.199096; PUBMED: 28400828]

\section{Navali 2016 \{published data only\}}

Navali N, Gassemzadeh A, Farzadi L, Abdollahi S, Nouri M, Hamdi K, et al. Intrauterine administration of hCG immediately after oocyte retrieval and the outcome of ICSI: a randomized controlled trial. Human Reproduction 2016;31(11):2520-6. [DOI: doi:10.1093/humrep/dew236; PUBMED: 27680029]

\section{Santibañez 2014 \{published data only\}}

Santibañez A, García J, Pashkova O, Colín O, Castellanos G, Sánchez AP, et al. Effect of intrauterine injection of human chorionic gonadotropin before embryo transfer on clinical pregnancy rates from in vitro fertilisation cycles: a prospective study. Reproductive Biology and Endocrinology 2014;12:9. [DOI: 10.1186/1477-7827-12-9; PUBMED: 24476536] 
Singh 2014 \{published data only\}

Singh R, Singh M. Intra-uterine administration of human chorionic gonadotrophin (hCG) before embryo transfer in recurrent implantation failure (RIF) patients improves implantation and pregnancy rates in IVF-ICSI cycles. Human Reproduction 2014;29(Suppl 1):i79.

\section{Wirleitner 2015a \{published data only\}}

Wirleitner B, Schuff M, Vanderzwalmen P, Stecher A, Okhowat J, Hradecký $L$, et al. Intrauterine administration of human chorionic gonadotropin does not improve pregnancy and life birth rates independently of blastocyst quality: a randomised prospective study. Reproductive Biology and Endocrinology 2015;13:70. [DOI: 10.1186/s12958-015-0069-1; PUBMED: 26141379]

\section{Wirleitner 2015b \{published data only\}}

Wirleitner B, Schuff M, Vanderzwalmen P, Stecher A, Hradecky L, Kohoutek T, et al. 0-182 - the usefulness of intrauterine hCG administration prior to blastocyst transfer in IVF-patients $\geq 38$ years. Human Reproduction 2015;30(Suppl 1):i79-i80. [DOI: 10.1093/humrep/30.Supplement_1.1]

\section{Zarei 2014 \{published data only\}}

Zarei A, Parsanezhad ME, Younesi M, Alborzi S, Zolghadri J, Samsami A, et al. Intrauterine administration of recombinant human chorionic gonadotropin before embryo transfer on outcome of in vitro fertilization/ intracytoplasmic sperm injection: a randomized clinical trial. Iranian Journal of Reproductive Medicine 2014;12:1-6. [PUBMED: 24799855]

\section{References to studies excluded from this review}

\section{Dieamant 2016 \{published data only\}}

Dieamant F, Oliveira JBA, Petersen CG, Mauri AL, Vagnini LD, Renzi A, et al. Can the intrauterine infusion of hCG improve ART outcomes? Unsafe and conflicting results in meta-analyses. Human Reproduction 2016;31(Suppl 1):i250. [DOI: 10.1093/ humrep/31.Supplement_1.1]

\section{Giuliani 2015 \{published data only\}}

Giuliani E, Olson M, Strug M, Young J, Shavell V, Dodds W, et al. Intrauterine hCG infusion affects the distribution of natural killer cells in the endometrium of fertile oocyte donors. Fertility and Sterility 2015;104:e149-e150.

\section{Huang 2017 \{published data only\}}

Huang P, Wei L, Li X, Qin A. Effects of intrauterine perfusion of human chorionic gonadotropin in women with different implantation failure numbers. American Journal of Reproductive Immunology 2017;79:e12809. [DOI: 10.1111/aji.12809; PUBMED: 29288552]

\section{Janati 2013 \{published data only\}}

Janati S, Dehghani Firouzabadi R, Mohseni F, Razi MH. Evaluation of effect of intrauterine human chorionic gonadotropin injection before embryo transfer in implantation and pregnancy rate in infertile patients and comparison with conventional embryo transfer in IVF/ICSI/ET cycles. Iranian Journal of Reproductive Medicine 2013;11:67-8.

\section{Jeong 2013 \{published data only\}}

Jeong HJ, Ryu MJ, Kim HM, Lee HS, Lee JH, Chung MK. Intrauterine injection of hCG before embryo transfer improves the clinical pregnancy rate and implantation rate in the patients with repeated implantation failure. Proceedings of the 5th IVI International Congress; 2013 Apr 4-6; Seville, Spain. http:// www.comtecmed.com/ivi/2013/Uploads/Editor/abstract_56.pdf 2013. [http://www.comtecmed.com/ivi/2013/Uploads/Editor/ abstract_56.pdf]

\section{Kanter 2017 \{published data only\}}

Kanter JR, Pollitzer C, Rao AS, Servy EJ, Massey J, GavrilovaJordan L. Intrauterine injection of hCG to enhance embryo implantation. Fertility and Sterility 2017;108:e370-e371.

\section{Li 2013 \{published data only\}}

Li T, Wang X, Yue C, Zhang J, Huang R, Liang X, et al. Intrauterine injection of human chorionic gonadotropin improves the pregnancy rates in in-vitro fertilizationembryo transfer cycles of repeated failures. Journal of Clinicians (Electronic Edition) 2013;7:3862-5. [DOI: 10.3877/ cma.j.issn.1674-0785.2013.09.0102]

\section{Osman 2016 \{published data only\}}

Osman A, Pundir J, Elsherbini M, Dave S, El-Toukhy T, Khalaf Y. The effect of intrauterine HCG injection on IVF outcome: a systematic review and meta-analysis. Reproductive BioMedicine Online 2016;33:350-9. [DOI: 10.1016/j.rbmo.2016.05.010; PUBMED: 27317131$]$

\section{Rebolloso 2013 \{published data only\}}

Rebolloso MM, Rosales De Leon JC, Galache Vega P, SantosHaliscak R, Diaz-Spindola P, Gonzalez Vega O. Do intrauterine injection of human chorionic gonadotropin (hCG) before embryo transfer increases implantation and pregnancy rates in patients undergoing in vitro fertilization?. Fertility and Sterility 2013;100:S289. [DOI: 10.1016/j.fertnstert.2013.07.1082]

\section{Riboldi 2013 \{published data only\}}

Riboldi M, Barros B, Piccolomini M, Alegretti JR, Motta ELA, Serafini PC. Does the intrauterine administration of rhCG before vitrified blastocysts transfer improves the potential of pregnancies when using blastocysts of inferior morphological grading?. Fertility and Sterility 2013;100:S289. [DOI: 10.1016/ j.fertnstert.2013.07.1080]

\section{Strug 2016 \{published data only\}}

Strug MR, Su R, Young JE, Dodds WG, Shavell VI, Díaz-Gimeno P, et al. Intrauterine human chorionic gonadotropin infusion in oocyte donors promotes endometrial synchrony and induction of early decidual markers for stromal survival: a randomized clinical trial. Human Reproduction 2016;31:1552-61. [DOI: 10.1093/humrep/dew080; PUBMED: 27122490]

\section{Volovsky 2016 \{published data only\}}

Volovsky M, Healey M, MacLachlan VB, Vollenhoven BJ. Intrauterine human chorionic gonadotropin (hCG) infusion prior to embryo transfer (ET) may be detrimental to pregnancy rate. Fertility and Sterility 2016;106:e52. 
Ye 2015 \{published data only\}

Ye H, Hu J, He W, Zhang Y, Li C. The efficacy of intrauterine injection of human chorionic gonadotropin before embryo transfer in assisted reproductive cycles: meta-analysis. Journal of International Medical Research 2015;43:738-46. [DOI: 0.1177/0300060515592903; PUBMED: 26359294]

\section{References to studies awaiting assessment}

Badehnoosh 2014 \{published data only\}

Badehnoosh B, Mohammadzadeh A, Sadeghi M, Akhondi M, Kazemnejad S, Sadaei-Jahromi N, et al. O-27 - the effects of intrauterine injection of human chorionic gonadotropin (hCG) before embryo transfer on the implantation rate in the intracytoplasmic sperm injection (ICSI) program. Iranian Journal of Reproductive Medicine 2014;12(Suppl 1):10-11.

Bhat 2014 \{published data only (unpublished sought but not used)\} Bhat VV, Dutta I, Dutta DK, Gcitha MD. Outcome of intrauterine injection of human chorionic gonadotropin before embryo transfer in patients with previous IVF/ICSI failure: a randomized study. Journal of South Asian Federation of Obstetrics and Gynaecology 2014;6(1):15-7. [DOI: 10.5005/jpjournals-10006-1259]

\section{References to ongoing studies}

IRCT2017041733486N1 \{published data only\}

Evaluation effect of intrauterine injection of human chorionic gonadotropin before embryo transfer on implantation rate and pregnancy rate in frozen cycles on IVF/ICSI. Ongoing study October 2017.

\section{NCT02668965 \{published data only\}}

The effects of intrauterine infusion of hCG at the time of embryo transfer. Ongoing study December 2015.

\section{NCT02825108 \{published data only\}}

Intrauterine injection of human chorionic gonadotropin injection (hCG) before embryo transfer on pregnancy outcomes in frozen embryo transfer cycles. Ongoing study January 2015.

\section{NCT02870855 \{published data only\}}

Effect of intrauterine injection of hCG on pregnancy outcome in repeated implantation failure patients. Ongoing study July 2017.

\section{NCT03238807 \{published data only\}}

Effect of intrauterine injection of hCG before ET on clinical outcomes in IVF/ICSI cycles. Ongoing study October 2017.

\section{Additional references}

\section{Abou-Setta 2014 \\ Abou-Setta AM, Peters LR, D'Angelo A, Sallam HN, Hart RJ, Al-Inany HG. Post-embryo transfer interventions for assisted reproduction technology cycles. Cochrane Database of Systematic Reviews 2014, Issue 8. [DOI: 10.1002/14651858.CD006567.pub3; PUBMED: 25157849]}

\section{Akhtar 2013}

Akhtar MA, Sur S, Raine-Fenning N, Jayaprakasan K, Thornton JG, Quenby S. Heparin for assisted reproduction. Cochrane Database of Systematic Reviews 2013, Issue 8. [DOI: 10.1002/14651858.CD009452.pub2; PUBMED: 23955506]

\section{Bourdiec 2013}

Bourdiec A, Bédard D, Rao CV, Akoum A. Human chorionic gonadotropin regulates endothelial cell responsiveness to interleukin 1 and amplifies the cytokine-mediated effect on cell proliferation, migration and the release of angiogenic factors. American Journal of Reproductive Immunology 2013;70:127-38. [DOI: 10.1111/aji.12080; PUBMED: 23351058]

\section{Brown 2010}

Brown J, Buckingham K, Abou-Setta AM, Buckett W. Ultrasound versus 'clinical touch' for catheter guidance during embryo transfer in women. Cochrane Database of Systematic Reviews 2010, Issue 1. [DOI: 10.1002/14651858.CD006107.pub3; PUBMED: 20091584]

\section{Cole 2010}

Cole LA. Biological functions of hCG and hCG-related molecules. Reproductive Biology and Endocrinology 2010;8:102. [DOI: 10.1186/1477-7827-8-102; PUBMED: 20735820]

\section{Craciunas 2014}

Craciunas L, Tsampras N, Fitzgerald C. Cervical mucus removal before embryo transfer in women undergoing in vitro fertilization/intracytoplasmic sperm injection: a systematic review and meta-analysis of randomized controlled trials. Fertility and Sterility 2014;101:1302-7. [DOI: 10.1016/ j.fertnstert.2014.01.047; PUBMED: 24602754]

\section{Derks 2009}

Derks RS, Farquhar C, Mol BW, Buckingham K, Heineman MJ. Techniques for preparation prior to embryo transfer. Cochrane Database of Systematic Reviews 2009, Issue 4. [DOI: 10.1002/14651858.CD007682.pub2; PUBMED: 19821435]

\section{Diedrich 2007}

Diedrich K, Fauser BC, Devroey P, Griesinger G, Evian Annual Reproduction (EVAR) Workshop Group. The role of the endometrium and embryo in human implantation. Human Reproduction Update 2007;13:365-77. [PUBMED: 17548368]

\section{GRADE 2013}

Schünemann H, Brożek J, Guyatt G, Oxman A. GRADE Handbook for Grading Quality of Evidence and Strength of Recommendations. The GRADE Working Group, 2013.

\section{GRADEpro 2015 [Computer program]}

McMaster University. GRADEpro GDT: GRADEpro Guideline Development Tool. Hamilton (ON): McMaster University, 2015.

\section{Higgins 2011}

Higgins JPT, Green S. Cochrane Handbook for Systematic Reviews of Interventions Version 5.1.0 (updated March 2011). The Cochrane Collaboration, 2011. Available from www.cochrane-handbook.org. 


\section{Kayisli 2003}

Kayisli UA, Selam B, Guzeloglu-Kayisli O, Demir R, Arici A. Human chorionic gonadotropin contributes to maternal immunotolerance and endometrial apoptosis by regulating FasFas ligand system. Journal of Immunology 2003;171:2305-13. [PUBMED: 12928375]

\section{Kupka 2014}

Kupka MS, Ferraretti AP, de Mouzon J, Erb K, D'Hooghe T, Castilla JA, et al. Assisted reproductive technology in Europe, 2010: results generated from European registers by ESHRE. Human Reproduction 2014;29:2099-113. [DOI: 10.1093/humrep/ deu175; PUBMED: 25069504]

\section{Licht 1998}

Licht P, Lösch A, Dittrich R, Neuwinger J, Siebzehnrübl E, Wildt L. Novel insights into human endometrial paracrinology and embryo-maternal communication by intrauterine microdialysis. Human Reproduction Update 1998;4:532-8. [PUBMED: 10027606]

\section{Mansour 1990}

Mansour R, Aboulghar M, Serour G. Dummy embryo transfer: a technique that minimizes the problems of embryo transfer and improves the pregnancy rate in human in vitro fertilization. Fertility and Sterility 1990;54:678-81. [PUBMED: 2209889]

\section{Nastri 2012}

Nastri CO, Gibreel A, Raine-Fenning N, Maheshwari A, Ferriani RA, Bhattacharya $S$, et al. Endometrial injury in women undergoing assisted reproductive techniques. Cochrane Database of Systematic Reviews 2012, Issue 7. [CENTRAL: CD009517; DOI: 10.1002/14651858.CD009517.pub2; PUBMED: 22786529]

\section{Racicot 2014}

Racicot KE, Wünsche V, Auerbach B, Aldo P, Silasi M, Mor G. Human chorionic gonadotropin enhances trophoblastepithelial interaction in an in vitro model of human implantation. Reproductive Sciences 2014;21:1274-80. [DOI: 10.1177/1933719114522553; PUBMED: 24520082]

\section{CHARACTERISTICS OF STUDIES}

Characteristics of included studies [ordered by study ID]

\section{RevMan 2014 [Computer program]}

The Nordic Cochrane Centre, The Cochrane Collaboration. Review Manager (RevMan). Version 5.3. Copenhagen: The Nordic Cochrane Centre, The Cochrane Collaboration, 2014.

\section{Schoolcraft 2001}

Schoolcraft WB, Surrey ES, Gardner DK. Embryo transfer: techniques and variables affecting success. Fertility and Sterility 2001;76:863-70. [PUBMED: 11704102]

\section{Siristatidis 2016}

Siristatidis CS, Basios G, Pergialiotis V, Vogiatzi P. Aspirin for in vitro fertilisation. Cochrane Database of Systematic Reviews 2016, Issue 11. [DOI: 10.1002/14651858.CD004832.pub4]

\section{Zegers-Hochschild 2009}

Zegers-Hochschild F, Adamson GD, de Mouzon J, Ishihara O, Mansour R, Nygren K, et al. The International Committee for Monitoring Assisted Reproductive Technology (ICMART) and the World Health Organization (WHO) Revised Glossary on ART Terminology, 2009. Human Reproduction 2009;24:2683-7. [DOI: 10.1093/humrep/dep343; PUBMED: 19801627]

\section{References to other published versions of this review Craciunas 2015}

Craciunas L, Tsampras N, Coomarasamy A, Raine-Fenning N. Intrauterine administration of human chorionic gonadotropin (hCG) for subfertile women undergoing assisted reproduction. Cochrane Database of Systematic Reviews 2015, Issue 2. [DOI: 10.1002/14651858.CD011537]

\section{Craciunas 2016}

Craciunas L, Tsampras N, Coomarasamy A, Raine-Fenning N. Intrauterine administration of human chorionic gonadotropin (hCG) for subfertile women undergoing assisted reproduction. Cochrane Database of Systematic Reviews 2016, Issue 5. [DOI: 10.1002/14651858.CD011537.pub2; PUBMED: 27195724]

* Indicates the major publication for the study

Aaleyasin 2015

\begin{tabular}{ll}
\hline Methods & Design: 2-arm parallel RCT \\
\hline Participants & Number: 483 \\
& Women's age (mean years; experimental vs control): 29.1 vs 28.7 \\
& Inclusion criteria: all infertile women who were candidates for the first IVF/ICSI \\
& $\begin{array}{l}\text { Exclusion criteria: aged > } 40 \text { years; history of percutaneous epididymal sperm aspiration; testicular } \\
\text { sperm extraction; myomectomy; hydrosalpinx; presence of uterine fibroma with the pressure effect on } \\
\text { endometrium; endometriosis; azoospermia }\end{array}$
\end{tabular}

Ovarian controlled hyperstimulation: long $\mathrm{GnRH}$ agonist protocol 
Aaleyasin 2015 (Continued)

\section{Fertilisation: ICSI}

Stage of the embryo at transfer: cleavage

Embryo processing: fresh

Number of embryos transferred (mean; experimental vs control): 2.8 vs 2.9

Interventions

Experimental ( $\mathbf{n}=\mathbf{2 4 0}$ ): hCG $500 \mathrm{IU}$ in a volume of $50 \mu \mathrm{L}$ tissue culture media (Vitrolife, Göteborg, Sweden) was injected into the uterus 5 to 7 minutes before ET

Control (n = 243): $50 \mu \mathrm{L}$ tissue culture media (Vitrolife, Göteborg, Sweden) instead of hCG

\begin{tabular}{|c|c|c|}
\hline Outcomes & \multicolumn{2}{|c|}{ Clinical pregnancy, miscarriage, live birth, intrauterine death } \\
\hline \multirow[t]{6}{*}{ Notes } & \multicolumn{2}{|c|}{ Location: Shariati Teaching Hospital, Tehran, Iran } \\
\hline & \multicolumn{2}{|c|}{ Period: January 2011 to July 2012} \\
\hline & \multicolumn{2}{|c|}{ Power calculation: yes } \\
\hline & \multicolumn{2}{|c|}{ Funding: not mentioned } \\
\hline & \multicolumn{2}{|c|}{ Trial registration: not mentioned and not found } \\
\hline & \multicolumn{2}{|c|}{ Publication type: full text } \\
\hline \multicolumn{3}{|l|}{ Risk of bias } \\
\hline Bias & Authors' judgement & Support for judgement \\
\hline $\begin{array}{l}\text { Random sequence genera- } \\
\text { tion (selection bias) }\end{array}$ & Low risk & Computer-generated list \\
\hline $\begin{array}{l}\text { Allocation concealment } \\
\text { (selection bias) }\end{array}$ & Low risk & $\begin{array}{l}\text { A technician, not belonging to the study personnel, prepared and coded drugs } \\
\text { according to the list. }\end{array}$ \\
\hline $\begin{array}{l}\text { Blinding of participants } \\
\text { and personnel (perfor- } \\
\text { mance bias) } \\
\text { All outcomes }\end{array}$ & Low risk & $\begin{array}{l}\text { All participants and clinical care providers were blinded to the list until the end } \\
\text { of the study. }\end{array}$ \\
\hline $\begin{array}{l}\text { Blinding of outcome as- } \\
\text { sessment (detection bias) } \\
\text { All outcomes }\end{array}$ & Low risk & $\begin{array}{l}\text { All participants and clinical care providers were blinded to the list until the end } \\
\text { of the study. }\end{array}$ \\
\hline $\begin{array}{l}\text { Incomplete outcome data } \\
\text { (attrition bias) } \\
\text { All outcomes }\end{array}$ & Low risk & Zero women were lost to follow-up. \\
\hline $\begin{array}{l}\text { Selective reporting (re- } \\
\text { porting bias) }\end{array}$ & Low risk & Reported on all important outcomes \\
\hline Other bias & Low risk & Similar baseline characteristics between groups after randomisation \\
\hline
\end{tabular}

Methods Design: 2-arm parallel RCT


Cambiaghi 2013 (Continued)

Participants

Number: 44

Women's age (mean years; experimental vs control): not mentioned

Inclusion criteria: endometrial thickness $>7 \mathrm{~mm}$ on the day the donor received hCG and at least 2

blastocysts on the day of ET

Exclusion criteria: not mentioned

Ovarian controlled hyperstimulation: donor oocytes, protocol not mentioned

Fertilisation: not mentioned

Stage of the embryo at transfer: blastocyst

Embryo processing: fresh

Number of embryos transferred: not mentioned (likely 2, from inclusion criteria)

Interventions

Experimental ( $\mathbf{n}=\mathbf{2 2})$ : intrauterine injection of hCG 500 IU 6 hours before ET

Control ( $\mathbf{n}=\mathbf{2 2})$ : ET without any pre-intrauterine injection

\begin{tabular}{ll}
\hline Outcomes & Clinical pregnancy \\
\hline Notes & Location: Instituto Paulista de Ginecologia, Obstetricia e Medicina Reproducao, Sao Paulo, Brazil \\
Period: January to December 2012 \\
Power calculation: no \\
Funding: not mentioned \\
Trial registration: not mentioned and not found \\
Publication type: abstract
\end{tabular}

\section{Risk of bias}

\begin{tabular}{lll}
\hline Bias & Authors' judgement & Support for judgement \\
\hline $\begin{array}{l}\text { Random sequence genera- } \\
\text { tion (selection bias) }\end{array}$ & Low risk & Computer-based randomisation \\
\hline $\begin{array}{l}\text { Allocation concealment } \\
\text { (selection bias) }\end{array}$ & Unclear risk & Allocation concealment not mentioned \\
\hline $\begin{array}{l}\text { Blinding of participants } \\
\text { and personnel (perfor- } \\
\text { mance bias) }\end{array}$ & High risk & Not mentioned \\
$\begin{array}{l}\text { All outcomes } \\
\text { Blinding of outcome as- } \\
\text { sessment (detection bias) } \\
\begin{array}{l}\text { All outcomes } \\
\text { Incomplete outcome data }\end{array}\end{array}$ & Low risk & Not mentioned, but unlikely to induce bias \\
$\begin{array}{l}\text { (attrition bias) } \\
\text { All outcomes }\end{array}$ & & \\
\hline
\end{tabular}

Intrauterine administration of human chorionic gonadotropin (hCG) for subfertile women undergoing assisted reproduction (Review) 
Cambiaghi 2013 (Continued)

Selective reporting (re- Unclear risk No reporting on adverse events, miscarriage, or live birth porting bias)

Other bias Unclear risk No reporting on baseline characteristics between groups

Dehghani Firouzabadi 2016

\begin{tabular}{ll}
\hline Methods & Design: 3-arm parallel RCT \\
\hline Participants & Number: 159 \\
& Women's age: 20 to 40 years \\
& Inclusion criteria: women aged 20 to 40 years with a male factor or unexplained infertility and basal \\
& FSH < 12 who had undergone assisted reproduction \\
& Exclusion criteria: azoospermia; presence of uterine myoma; endometriosis; hydrosalpinges; previ- \\
& ous IVF/ICSI trials (successful or unsuccessful); history of endocrine disease such as diabetes and thy- \\
roid dysfunction; previous history of hysteroscopic operation due to submucosal myoma; intrauterine \\
synechia
\end{tabular}

Ovarian controlled hyperstimulation: antagonist protocol

Fertilisation: ICSI

Stage of the embryo at transfer: cleavage

Embryo processing: fresh

Number of embryos transferred: 1 to 3

$\begin{array}{ll}\text { Interventions } & \text { Experimental }(\mathbf{n}=\mathbf{5 3}) \text { : hCG } 500 \mathrm{IU}(40 \mu \mathrm{L}) \text { intrauterine injection } 7 \text { minutes before ET } \\ & \text { Experimental }(\mathbf{n}=\mathbf{5 3}) \text { : hCG } 1000 \mathrm{IU}(40 \mu \mathrm{L}) \text { intrauterine injection } 7 \text { minutes before ET } \\ \text { Control }(\mathbf{n}=\mathbf{5 3}) \text { : nothing before ET }\end{array}$

Outcomes Clinical pregnancy, miscarriage

Notes Location: Research and Clinical Center for Infertility, Shahid Sadoughi University of Medical Sciences, Yazd, Iran

Period: April 2012 to March 2013

Power calculation: not mentioned

Funding: not mentioned

Trial registration: IRCT2012091310328N3

Publication type: full text

\section{Risk of bias}

\begin{tabular}{lll}
\hline Bias & Authors' judgement & Support for judgement \\
\hline $\begin{array}{l}\text { Random sequence genera- } \\
\text { tion (selection bias) }\end{array}$ & Low risk & $\begin{array}{l}\text { Liable women were randomly assigned to 2 test groups in the ratio of } 1: 1 \text { or to } \\
\text { a control group according to computer-generated random numbers }(\mathrm{n}=53) .\end{array}$ \\
\hline
\end{tabular}


Dehghani Firouzabadi 2016 (Continued)

Allocation concealment Unclear risk Allocation concealment not mentioned
(selection bias)

Blinding of participants High risk $\quad$ Not blinded
and personnel (perfor-
mance bias)
All outcomes

Blinding of outcome as- Low risk Not blinded, but unlikely to induce bias

sessment (detection bias)

All outcomes

\begin{tabular}{lll}
\hline $\begin{array}{l}\text { Incomplete outcome data } \\
\text { (attrition bias) } \\
\text { All outcomes }\end{array}$ & Low risk & Data reported on all randomised participants \\
\hline $\begin{array}{l}\text { Selective reporting (re- } \\
\text { porting bias) }\end{array}$ & Unclear risk & No live birth data \\
\hline Other bias & Low risk & Similar baseline characteristics between groups after randomisation \\
\hline
\end{tabular}

\section{Eskandar 2016}

\begin{tabular}{ll}
\hline Methods & Design: 2 -arm parallel RCT \\
\hline Participants & Wumber: 240 \\
& Inclusion criteria: women undergoing embryo transfer \\
& Exclusion criteria: not mentioned \\
& Ovarian controlled hyperstimulation: not mentioned \\
& Fertilisation: IVF and ICSI \\
& Stage of the embryo at transfer: not mentioned, assumed cleavage (day 3) based on other publica- \\
tions from the same IVF unit & Embryo processing: not mentioned \\
& Number of embryos transferred: 2 to 3 \\
\end{tabular}

Interventions $\quad$ Experimental $(\mathbf{n}=\mathbf{1 3 9})$ : $500 \mathrm{IU}$ of hCG intrauterine 10 minutes before ET

Control ( $\mathbf{n}=\mathbf{1 0 1})$ : ET without any pre-intrauterine injection

\begin{tabular}{ll}
\hline Outcomes & Clinical pregnancy \\
\hline Notes & Location: Saudi Center for Assisted Reproduction, Abha, Saudi Arabia \\
& Period: not mentioned \\
& Power calculation: not mentioned \\
& Funding: not mentioned \\
& Trial registration: not mentioned and not found
\end{tabular}


Eskandar 2016 (Continued)

Publication type: abstract

\section{Risk of bias}

\begin{tabular}{|c|c|c|}
\hline Bias & Authors' judgement & Support for judgement \\
\hline $\begin{array}{l}\text { Random sequence genera- } \\
\text { tion (selection bias) }\end{array}$ & Low risk & $\begin{array}{l}\text { Participants were divided randomly into } 2 \text { groups by a computer-based pro- } \\
\text { gramme. }\end{array}$ \\
\hline $\begin{array}{l}\text { Allocation concealment } \\
\text { (selection bias) }\end{array}$ & Unclear risk & Allocation concealment was not mentioned. \\
\hline $\begin{array}{l}\text { Blinding of participants } \\
\text { and personnel (perfor- } \\
\text { mance bias) } \\
\text { All outcomes }\end{array}$ & High risk & Blinding was not mentioned. \\
\hline $\begin{array}{l}\text { Blinding of outcome as- } \\
\text { sessment (detection bias) } \\
\text { All outcomes }\end{array}$ & Low risk & Blinding not mentioned, but unlikely to induce bias \\
\hline $\begin{array}{l}\text { Incomplete outcome data } \\
\text { (attrition bias) } \\
\text { All outcomes }\end{array}$ & Unclear risk & Very brief reporting on results \\
\hline $\begin{array}{l}\text { Selective reporting (re- } \\
\text { porting bias) }\end{array}$ & Unclear risk & No reporting on adverse events, live birth, or miscarriage \\
\hline Other bias & Low risk & Similar baseline characteristics between groups after randomisation \\
\hline
\end{tabular}

Hong 2014

\begin{tabular}{ll}
\hline Methods & Design: 2-arm parallel RCT \\
\hline Purticipants & Women's age (mean years; experimental vs control): 35.0 vs 35.1 \\
& Inclusion criteria: all participants undergoing fresh or frozen ET within the ART programme when the \\
female partner was younger than 43 years of age \\
Exclusion criteria: women could not be simultaneously participating in another prospective clinical \\
trial at the centre, but no other inclusion/exclusion criteria were applied \\
Ovarian controlled hyperstimulation: not mentioned \\
Fertilisation: not mentioned \\
Stage of the embryo at transfer: blastocyst \\
Embryo processing: fresh and frozen/thawed \\
Number of embryos transferred: 1 or 2 \\
Experimental (n = 148): endometrial infusion of $20 \mu \mathrm{L}$ ET media (synthetic serum substitute and \\
MediCult BlastAssist, Origio) laden with 500 IU of purified urinary placental hCG (Novarel, Ferring Phar- \\
maceuticals) < 3 minutes before ET \\
Control (152): endometrial infusion of $20 \mu \mathrm{L}$ ET media only \\
\hline Interventions
\end{tabular}


Hong 2014 (Continued)

Outcomes Miscarriage and clinical pregnancy (converted from ongoing pregnancy)

Notes Location: Reproductive Medicine Associates of New Jersey, Princeton, New Jersey, USA

Period: August 2012 to December 2013

Power calculation: yes, but not met (778 embryos required, 473 embryos transferred)

Funding: not mentioned

Trial registration: NCT01643993

Publication type: full text

\section{Risk of bias}

\begin{tabular}{|c|c|c|}
\hline Bias & Authors' judgement & Support for judgement \\
\hline $\begin{array}{l}\text { Random sequence genera- } \\
\text { tion (selection bias) }\end{array}$ & Low risk & $\begin{array}{l}\text { A random number function was used to create variable blocks of } 4 \text { to } 8 \text {, with } \\
\text { participants assigned to the } 2 \text { groups in a } 1: 1 \text { allocation. }\end{array}$ \\
\hline $\begin{array}{l}\text { Allocation concealment } \\
\text { (selection bias) }\end{array}$ & Low risk & $\begin{array}{l}\text { Allocation concealment was achieved with sequentially numbered, opaque, } \\
\text { sealed envelopes. }\end{array}$ \\
\hline $\begin{array}{l}\text { Blinding of participants } \\
\text { and personnel (perfor- } \\
\text { mance bias) } \\
\text { All outcomes }\end{array}$ & Low risk & $\begin{array}{l}\text { Both the physician performing the transfer and the participants were blinded } \\
\text { to the assigned treatment group throughout the entirety of the study. }\end{array}$ \\
\hline $\begin{array}{l}\text { Blinding of outcome as- } \\
\text { sessment (detection bias) } \\
\text { All outcomes }\end{array}$ & Low risk & Not mentioned, but unlikely to induce bias \\
\hline $\begin{array}{l}\text { Incomplete outcome data } \\
\text { (attrition bias) } \\
\text { All outcomes }\end{array}$ & Low risk & No loss to follow-up \\
\hline $\begin{array}{l}\text { Selective reporting (re- } \\
\text { porting bias) }\end{array}$ & Unclear risk & No reports on live births and adverse events \\
\hline Other bias & Unclear risk & $\begin{array}{l}25 \text { participants declined to participate for various reasons after randomisa- } \\
\text { tion. }\end{array}$ \\
\hline
\end{tabular}

Hosseini 2016

Methods Design: 2-arm parallel RCT

Participants Number: 100

Women's age (mean years; experimental vs control): 30.5 vs 31.3

Inclusion criteria: women undergoing assisted reproduction

Exclusion criteria: history of uterine surgery such as myomectomy; history of recurrent miscarriage; known hydrosalpinx, endometrioma, or endometriosis 
Ovarian controlled hyperstimulation: frozen/thawed cycles; preparation of endometrium initiated with hormone replacement protocol, which involved administration of oestrogen, followed by progesterone without ovarian downregulation

Fertilisation: not mentioned

Stage of the embryo at transfer: $90 \%+$ cleavage, $<10 \%$ blastocysts

Embryo processing: frozen/thawed Number of embryos transferred: 2 to 3

Interventions

Experimental ( $\mathbf{n}=\mathbf{5 0}$ ): case group received intrauterine injection of $40 \mu \mathrm{L}$ of a 5000-unit hCG vial (Choriomon, IBSA, Lugano) mixed with $0.4 \mathrm{~mL}$ of IMSI-type media (equivalent to $500 \mathrm{hCG}$ units) through Labotect catheter (Labotect, Labor-Technik-Gottingen GmbH, Germany). Seven minutes later, embryo transfer was performed with a sterile Labotect catheter, guided by abdominal ultrasound at 1 to $1.5 \mathrm{~cm}$ from uterine fundus.

Control $(\mathbf{n}=\mathbf{5 0})$ : in the control group, embryo transfer was carried out with no intervention

\begin{tabular}{ll}
\hline Outcomes & Clinical pregnancy, miscarriage, still birth \\
\hline Notes & Location: Al-Zahra Hospital Fertility Center, Tabriz, Iran \\
Period: May 2014 to April 2015 \\
Power calculation: no \\
Funding: not mentioned \\
Trial registration: not mentioned \\
Publication type: full text
\end{tabular}

\section{Risk of bias}

\begin{tabular}{|c|c|c|}
\hline Bias & Authors' judgement & Support for judgement \\
\hline $\begin{array}{l}\text { Random sequence genera- } \\
\text { tion (selection bias) }\end{array}$ & Low risk & $\begin{array}{l}\text { During embryo transfer, participants were randomly divided (according to ta- } \\
\text { ble of random numbers) into control and case groups ( } 50 \text { patients each). }\end{array}$ \\
\hline $\begin{array}{l}\text { Allocation concealment } \\
\text { (selection bias) }\end{array}$ & Unclear risk & Allocation concealment was not mentioned. \\
\hline $\begin{array}{l}\text { Blinding of participants } \\
\text { and personnel (perfor- } \\
\text { mance bias) } \\
\text { All outcomes }\end{array}$ & High risk & $\begin{array}{l}\text { Blinding was not possible owing to the nature of the intervention (control } \\
\text { group received no placebo). }\end{array}$ \\
\hline $\begin{array}{l}\text { Blinding of outcome as- } \\
\text { sessment (detection bias) } \\
\text { All outcomes }\end{array}$ & Low risk & Not blinded, but unlikely to induce bias \\
\hline $\begin{array}{l}\text { Incomplete outcome data } \\
\text { (attrition bias) } \\
\text { All outcomes }\end{array}$ & Low risk & All participants were accounted for by outcome measures. \\
\hline $\begin{array}{l}\text { Selective reporting (re- } \\
\text { porting bias) }\end{array}$ & Unclear risk & No live birth data \\
\hline Other bias & Low risk & Similar baseline characteristics between groups after randomisation \\
\hline
\end{tabular}


Huang 2016

Methods

Participants
Design: 3-arm parallel RCT, only data from control group (not placebo) used

Number: 161 total, 115 used for comparison

Women's age (mean years; experimental vs control): 33.95 vs 33.08

Inclusion criteria: 2 instances of failed transfer of good-quality embryos; undergoing FET cycles; aged 38 years; body mass index (BMI) of 18 to 24 ; normal endometrial thickness ( 8 to $16 \mathrm{~mm}$ ); frozen preservation of $\geq 2$ embryos, with at least 1 good-quality embryo

Exclusion criteria: diseases such as endometrial polyps, intrauterine adhesion, or uterine submucosal myomas, which might cause endometrial abnormalities; adenomyosis; hydropic fallopian tubes, PCOS, or endometriosis $\geq$ stage III

Ovarian controlled hyperstimulation: frozen/thawed cycles; preparation of endometrium was conducted with letrozole and progesterone

Fertilisation: not mentioned

Stage of the embryo at transfer: cleavage

Embryo processing: frozen/thawed

Number of embryos transferred: 2

Experimental ( $\mathbf{n}=\mathbf{6 5})$ : the perfusion group received 1000 IU of hCG (Lizhu, Zhuhai, China) mixed with 1 $\mathrm{mL}$ of normal saline via intrauterine injection 3 days before $E T$

Control ( $\mathbf{n}=\mathbf{5 0})$ : no intrauterine injection

\begin{tabular}{|c|c|c|}
\hline Outcomes & \multicolumn{2}{|c|}{ Clinical pregnancy, miscarriage } \\
\hline \multirow[t]{6}{*}{ Notes } & \multicolumn{2}{|c|}{$\begin{array}{l}\text { Location: Center of Reproductive Medicine of Liuzhou Maternity and Child Healthcare Hospital, } \\
\text { Guangxi Province, China }\end{array}$} \\
\hline & \multicolumn{2}{|c|}{ Period: January 2015 and December 2015} \\
\hline & \multicolumn{2}{|c|}{ Power calculation: no } \\
\hline & \multicolumn{2}{|c|}{ Funding: not mentioned } \\
\hline & \multicolumn{2}{|c|}{ Trial registration: not mentioned } \\
\hline & \multicolumn{2}{|c|}{ Publication type: full text } \\
\hline \multicolumn{3}{|l|}{ Risk of bias } \\
\hline Bias & Authors' judgement & Support for judgement \\
\hline $\begin{array}{l}\text { Random sequence genera- } \\
\text { tion (selection bias) }\end{array}$ & Low risk & $\begin{array}{l}\text { Randomisation via a computerised random digit generator based on patient } \\
\text { registration number in order of referral }\end{array}$ \\
\hline $\begin{array}{l}\text { Allocation concealment } \\
\text { (selection bias) }\end{array}$ & Unclear risk & Not mentioned \\
\hline $\begin{array}{l}\text { Blinding of participants } \\
\text { and personnel (perfor- } \\
\text { mance bias) } \\
\text { All outcomes }\end{array}$ & Unclear risk & Single-blinded mentioned, but not clear who was blinded \\
\hline
\end{tabular}


Huang 2016 (Continued)

Blinding of outcome as- Low risk Not mentioned, but unlikely to induce bias sessment (detection bias)

All outcomes

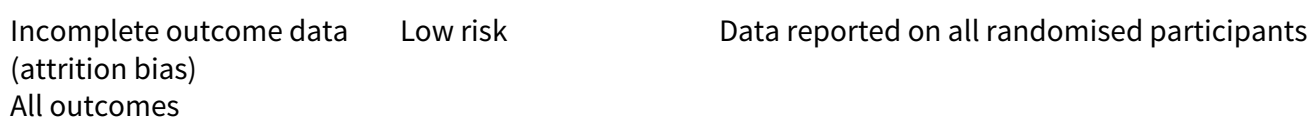

Selective reporting (re- Unclear risk No live birth data

Other bias Low risk Similar baseline characteristics between groups after randomisation

Kokkali 2014

\begin{tabular}{|c|c|}
\hline Methods & Design: 2-arm parallel RCT \\
\hline \multirow[t]{9}{*}{ Participants } & Number: 194 \\
\hline & Women's age (years): $>40$ \\
\hline & Inclusion criteria: women aged $>40$ years receiving donor eggs \\
\hline & Exclusion criteria: not mentioned \\
\hline & Ovarian controlled hyperstimulation: not mentioned \\
\hline & Fertilisation: not mentioned \\
\hline & Stage of the embryo at transfer: not mentioned \\
\hline & Embryo processing: not mentioned \\
\hline & Number of embryos transferred: not mentioned \\
\hline \multirow[t]{2}{*}{ Interventions } & Experimental ( $\mathbf{n = 9 7 ) : ~ i n t r a u t e r i n e ~ h C G ~} 500$ IU injection 7 minutes before ET \\
\hline & Control $(n=97):$ no intrauterine injection \\
\hline Outcomes & Clinical pregnancy \\
\hline \multirow[t]{6}{*}{ Notes } & Location: Genesis Athens Hospital, Centre for Human Reproduction, Athens, Greece \\
\hline & Period: July 2012 to September 2013 \\
\hline & Power calculation: no \\
\hline & Funding: Genesis Athens Clinic \\
\hline & Trial registration: not registered \\
\hline & Publication type: abstract \\
\hline
\end{tabular}

\section{Risk of bias}

\begin{tabular}{lll}
\hline Bias & Authors' judgement & Support for judgement \\
\hline $\begin{array}{l}\text { Random sequence genera- } \\
\text { tion (selection bias) }\end{array}$ & Low risk & $\begin{array}{l}\text { Randomisation was performed in a 1:1 fashion to 1 of 2 groups [...] prepared } \\
\text { from a computer-generated list. }\end{array}$ \\
\hline \hline
\end{tabular}


Kokkali 2014 (Continued)

$\begin{aligned} & \text { Allocation concealment Low risk } \\ & \text { (selection bias) }\end{aligned}$
opaque, sealed envelopes prepared from a computer-generated list.
(selection bias)

High risk

Not blinded

Blinding of participants

and personnel (perfor-

mance bias)

All outcomes

Blinding of outcome as-
sessment (detection bias) $\quad$ Low risk $\quad$ Not blinded, but unlikely to induce bias

(detection bias)

All outcomes

Incomplete outcome data Unclear risk $\quad$ Very brief reporting on results
(attrition bias)

All outcomes

Selective reporting (re- Unclear risk No reporting on live birth and adverse events
porting bias)

porting bias)

Other bias Unclear risk No reporting on baseline characteristics between groups

Leao 2013

\begin{tabular}{ll}
\hline Methods & Design: 2 -arm parallel RCT \\
\hline Participants & Wumber: 36 \\
& Inclusion criteria: women with 2 previous failures in IVF cycles with ET \\
& Exclusion criteria: not mentioned \\
& Ovarian controlled hyperstimulation: not mentioned \\
& Fertilisation: not mentioned \\
& Stage of the embryo at transfer: not mentioned \\
& Embryo processing: not mentioned \\
& Number of embryos transferred: not mentioned
\end{tabular}

Interventions Experimental $(\mathbf{n}=\mathbf{1 8})$ : intrauterine injection of hCG 500 IU 6 hours before ET

Control ( $\mathbf{n}=\mathbf{1 8})$ : women were forwarded straight to ET

\begin{tabular}{ll}
\hline Outcomes & Clinical pregnancy \\
\hline Notes & Location: IPGO, Sao Paulo, Brazil \\
& Period: January to December 2012 \\
& Power calculation: no \\
& Funding: not mentioned \\
& Trial registration: not mentioned and not found
\end{tabular}




\section{Risk of bias}

\begin{tabular}{|c|c|c|}
\hline Bias & Authors' judgement & Support for judgement \\
\hline $\begin{array}{l}\text { Random sequence genera- } \\
\text { tion (selection bias) }\end{array}$ & Unclear risk & Randomisation mentioned with no details \\
\hline $\begin{array}{l}\text { Allocation concealment } \\
\text { (selection bias) }\end{array}$ & Unclear risk & Allocation concealment not mentioned \\
\hline $\begin{array}{l}\text { Blinding of participants } \\
\text { and personnel (perfor- } \\
\text { mance bias) } \\
\text { All outcomes }\end{array}$ & High risk & Not mentioned \\
\hline $\begin{array}{l}\text { Blinding of outcome as- } \\
\text { sessment (detection bias) } \\
\text { All outcomes }\end{array}$ & Low risk & Not mentioned, but unlikely to induce bias \\
\hline $\begin{array}{l}\text { Incomplete outcome data } \\
\text { (attrition bias) } \\
\text { All outcomes }\end{array}$ & Unclear risk & Very brief reporting on results \\
\hline $\begin{array}{l}\text { Selective reporting (re- } \\
\text { porting bias) }\end{array}$ & Unclear risk & No reporting on adverse events, miscarriage, or live birth \\
\hline Other bias & High risk & $\begin{array}{l}\text { Number of participants in each arm was not reported, but was deduced based } \\
\text { on percentages and previous study by the same team }\end{array}$ \\
\hline
\end{tabular}

Mansour 2011

Methods Design: 2 RCTs within the same study analysed as 4-armed parallel RCT

Participants

Number: $280+215=495$

Women's age (mean years; experimental 100, 200 vs control; 500 vs control): 29 vs 28.5 vs 29.1 ; 28.3 vs 28.4

Inclusion criteria: women aged $<40$ years old with infertility due to male factor

Exclusion criteria: previous IVF/ICSI trials, including a successful trial; azoospermia; uterine myoma or previous myomectomy; endometriosis; presence of

hydrosalpinges

Ovarian controlled hyperstimulation: not mentioned

Fertilisation: ICSI

Stage of the embryo at transfer: cleavage

Embryo processing: fresh

Number of embryos transferred (mean; experimental 100, 200 vs control; 500 vs control): 2.9 vs 2.8 vs $2.9 ; 2.9$ vs 2.8 
Mansour 2011 (Continued)

Interventions
Experimental 100 ( $\mathbf{n}=\mathbf{9 2}$ ): $40 \mu \mathrm{L}$ of tissue culture medium (G-2 plus ref. 10132, Vitrolife, Göteborg, Sweden) containing hCG $100 \mathrm{IU}$ injected intrauterine approximately 7 minutes before ET

Experimental 200 ( $\mathbf{n}=93$ ): $40 \mu \mathrm{L}$ of tissue culture medium (G-2 plus ref. 10132, Vitrolife, Göteborg, Sweden) containing hCG 200 IU injected intrauterine approximately 7 minutes before ET

Experimental 500 ( $\mathbf{n}=\mathbf{1 0 8}$ ): $40 \mu \mathrm{L}$ of tissue culture medium (G-2 plus ref. 10132, Vitrolife, Göteborg, Sweden) containing hCG 500 IU injected intrauterine approximately 7 minutes before ET

Control ( $\mathbf{n}=95$ + 107): no intrauterine hCG injection before ET

\begin{tabular}{ll}
\hline Outcomes & Live birth, miscarriage, clinical pregnancy, ectopic pregnancy \\
\hline Notes & Location: The Egyptian IVF-ET Center, Cairo, Egypt \\
Period: January 2010 to January 2011 \\
Power calculation: yes, but not met \\
Funding: The Egyptian IVF-ET Center \\
Trial registration: NCT01030393 \\
Publication type: full text \\
Live birth rate was established by personal communication with study authors, June 2015. Study publi- \\
cation reported number of deliveries, which included 6 women who had stillbirths (3 in each group).
\end{tabular}

\section{Risk of bias}

\begin{tabular}{|c|c|c|}
\hline Bias & Authors' judgement & Support for judgement \\
\hline $\begin{array}{l}\text { Random sequence genera- } \\
\text { tion (selection bias) }\end{array}$ & Low risk & $\begin{array}{l}\text { Participants were randomised to } 2 \text { groups with the use of sealed dark en- } \\
\text { velopes. }\end{array}$ \\
\hline $\begin{array}{l}\text { Allocation concealment } \\
\text { (selection bias) }\end{array}$ & Unclear risk & $\begin{array}{l}\text { Allocation concealment not mentioned. Could explain different withdrawal } \\
\text { rates between groups }\end{array}$ \\
\hline $\begin{array}{l}\text { Blinding of participants } \\
\text { and personnel (perfor- } \\
\text { mance bias) } \\
\text { All outcomes }\end{array}$ & High risk & Not blinded \\
\hline $\begin{array}{l}\text { Blinding of outcome as- } \\
\text { sessment (detection bias) } \\
\text { All outcomes }\end{array}$ & Low risk & Not blinded, but unlikely to induce bias \\
\hline $\begin{array}{l}\text { Incomplete outcome data } \\
\text { (attrition bias) } \\
\text { All outcomes }\end{array}$ & Unclear risk & Women lost to follow-up live birth (similar numbers between groups) \\
\hline $\begin{array}{l}\text { Selective reporting (re- } \\
\text { porting bias) }\end{array}$ & Low risk & Reported on all important outcomes \\
\hline Other bias & High risk & $\begin{array}{l}\text { Interim analysis with change of protocol and premature ending of study. Rela- } \\
\text { tively high live birth rate in control group, reasons unclear }\end{array}$ \\
\hline
\end{tabular}


Mostajeran 2017

\begin{tabular}{|c|c|}
\hline Methods & Design: 2-arm parallel RCT \\
\hline \multirow[t]{4}{*}{ Participants } & Number: 100 \\
\hline & Women's age: mean $31.3 \pm 5.2$ years \\
\hline & $\begin{array}{l}\text { Inclusion criteria: women } 20 \text { to } 40 \text { years old with body mass index } 18 \text { to } 30 \mathrm{~kg} / \mathrm{m}^{2} \text { were eligible if they } \\
\text { were infertile owing to male factor, had a regular menstrual cycle of } 24 \text { to } 35 \mathrm{days} \text {, and were presumed } \\
\text { to be ovulatory }\end{array}$ \\
\hline & $\begin{array}{l}\text { Exclusion criteria: presence of polycystic ovary syndrome, with uterine pathologies, endometriosis, or } \\
\text { presence of hydrosalpinges and any endocrine disease or chronic systemic illness; azoospermia; histo- } \\
\text { ry of successful IVF or ICSI }\end{array}$ \\
\hline
\end{tabular}

Ovarian controlled hyperstimulation: not mentioned

Fertilisation: IVF and ICSI

Stage of the embryo at transfer: blastocyst

Embryo processing: frozen/thawed

Number of embryos transferred: 1 to 3

Interventions

Experimental $(\mathbf{n}=\mathbf{5 0})$ : injection of $700 \mathrm{IU}$ of intrauterine hCG (chorionic gonadotropin human, Darou Pakhsh Company, Iran) 10 minutes before embryo transfer

Control $(\mathbf{n}=\mathbf{5 0})$ : did not receive hCG before embryo transfer

\begin{tabular}{ll}
\hline Outcomes & Clinical pregnancy \\
\hline Notes & Location: Fertility and Infertility Center of Isfahan in Iran \\
& Period: September 2013 to April 2014 \\
& Power calculation: yes, but inadequate \\
& Funding: not mentioned \\
& Trial registration: not mentioned \\
& Publication type: full text \\
\hline
\end{tabular}

Risk of bias

\begin{tabular}{lll}
\hline Bias & Authors' judgement & Support for judgement \\
\hline $\begin{array}{l}\text { Random sequence genera- } \\
\text { tion (selection bias) }\end{array}$ & Low risk & $\begin{array}{l}\text { Participants were randomly divided into two 50 - member groups by random } \\
\text { allocation software. } \\
\text { Saghaei, 2004 }\end{array}$ \\
\hline $\begin{array}{l}\text { Allocation concealment } \\
\text { (selection bias) }\end{array}$ & Unclear risk & Not mentioned \\
\hline $\begin{array}{l}\text { Blinding of participants } \\
\begin{array}{l}\text { and personnel (perfor- } \\
\text { mance bias) }\end{array}\end{array}$ & Low risk & $\begin{array}{l}\text { Embryo transfer in both groups was done by the attending gynaecologist, who } \\
\text { All outcomes }\end{array}$ \\
\hline $\begin{array}{l}\text { Blinding of outcome as- } \\
\text { sessment (detection bias) } \\
\text { All outcomes }\end{array}$ & Low risk & Not blinded, but unlikely to induce bias \\
\hline
\end{tabular}


Mostajeran 2017 (Continued)

Incomplete outcome data Unclear risk 6 participants lost to follow-up

(attrition bias)

All outcomes

Selective reporting (re- Unclear risk $\quad$ No data on miscarriage or live birth
porting bias)

Other bias Low risk Similar baseline characteristics between groups after randomisation

Navali 2016

\begin{tabular}{ll}
\hline Methods & Design: 2 -arm parallel RCT \\
\hline Participants & Number: 158 \\
& Women's age (mean years; experimental vs control): $30.6 \mathrm{vs} 32$ \\
& Inclusion criteria: normal ovarian reserve (anti-Müllerian hormone $\geq 0.7 \mathrm{ng} / \mathrm{mL}$ ); age $\leq 41 \mathrm{years;}$ unde \\
& going ICSI and fresh ET; normal levels of thyroid-stimulating hormone and prolactin \\
& $\begin{array}{l}\text { Exclusion criteria: uncontrolled chronic maternal disease such as endocrinopathy and autoimmune } \\
\text { disease, severe endometriosis, severe hydrosalpinx, or non-obstructive azoospermia; high risk for se- } \\
\text { vere ovarian hyperstimulation syndrome (development of }>20 \text { follicles }>10 \text { mm at ovarian stimulation } \\
\text { or retrieval of }>16 \text { oocytes on the day of oocyte retrieval); morphological embryo deficiencies }\end{array}$
\end{tabular}

Ovarian controlled hyperstimulation: flexible antagonist protocol

Fertilisation: ICSI

Stage of the embryo at transfer: cleavage

Embryo processing: fresh

Number of embryos transferred: 2 to 3

Interventions Experimental $(\mathbf{n}=\mathbf{8 0}): 0.1 \mathrm{~mL}(500 \mathrm{IU} \mathrm{hCG})$ and $0.4 \mathrm{~mL}$ normal saline were pulled into an insulin syringe and injected into the uterus immediately after oocyte retrieval under general anaesthesia

Control ( $\mathbf{n = 7 8 ) : ~} 0.5 \mathrm{~mL}$ normal saline injected into the uterus at the same time as experimental group

Outcomes Clinical pregnancy, miscarriage, ectopic pregnancy

Notes Location: Reproductive Medical Center, Al-Zahra University Hospital, Tabriz University of Medical Sciences, Tabriz, Iran

Period: September 2015 to February 2016

Power calculation: yes, but not met

Funding: Women's Health Research Center, Tabriz University of Medical Sciences, Iran. No external funds were used.

Trial registration: IRCT201206165485N4

Publication type: full text

\section{Risk of bias}


Navali 2016 (Continued)

$\begin{array}{ll}\begin{array}{l}\text { Random sequence genera- } \\ \text { tion (selection bias) }\end{array} & \text { Low risk } \\ \end{array}$
tion (selection bias) tion was used to randomise participants.

\begin{tabular}{|c|c|c|}
\hline $\begin{array}{l}\text { Allocation concealment } \\
\text { (selection bias) }\end{array}$ & Low risk & $\begin{array}{l}\text { Treatment allocation was placed in a sealed, opaque envelope that was picked } \\
\text { up consecutively by an operating room technician during the oocyte retrieval } \\
\text { procedure. }\end{array}$ \\
\hline
\end{tabular}

\begin{tabular}{ll}
\hline $\begin{array}{l}\text { Blinding of participants } \\
\text { and personnel (perfor- }\end{array}$ & Low risk \\
anly the technician was aware of the participant's allocation; she prepared \\
and handed the intervention drug to the physician.
\end{tabular}
mance bias)

All outcomes

\begin{tabular}{|c|c|c|}
\hline $\begin{array}{l}\text { Blinding of outcome as- } \\
\text { sessment (detection bias) } \\
\text { All outcomes }\end{array}$ & Low risk & $\begin{array}{l}\text { Only the technician was aware of the participant's allocation; she prepared } \\
\text { and handed the intervention drug to the physician. }\end{array}$ \\
\hline $\begin{array}{l}\text { Incomplete outcome data } \\
\text { (attrition bias) } \\
\text { All outcomes }\end{array}$ & High risk & $\begin{array}{l}20(12 \%) \text { participants were lost to follow-up or were excluded following ran- } \\
\text { domisation. }\end{array}$ \\
\hline $\begin{array}{l}\text { Selective reporting (re- } \\
\text { porting bias) }\end{array}$ & Unclear risk & No data on live birth \\
\hline Other bias & Low risk & Similar baseline characteristics between groups after randomisation \\
\hline
\end{tabular}

Santibañez 2014

\begin{tabular}{|c|c|}
\hline Methods & Design: 2-arm parallel RCT \\
\hline \multirow[t]{9}{*}{ Participants } & Number: 210 \\
\hline & Women's age (mean years; experimental vs control): 36.4 vs 37.3 \\
\hline & Inclusion criteria: infertile women aged < 40 years who had an indication for an IVF/ICSI \\
\hline & Exclusion criteria: azoospermia \\
\hline & Ovarian controlled hyperstimulation: indicated based on individual participant characteristics \\
\hline & Fertilisation: IVF or ICSI \\
\hline & Stage of the embryo at transfer: cleavage \\
\hline & Embryo processing: fresh and frozen/thawed \\
\hline & Number of embryos transferred (mean): 2.1 \\
\hline \multirow[t]{2}{*}{ Interventions } & $\begin{array}{l}\text { Experimental ( } \mathbf{n}=\mathbf{1 0 1}): 20 \mu \mathrm{L} \text { of embryo culture medium (G-2, Vitrolife, Göteborg, Sweden) that con- } \\
\text { tained hCG } 500 \text { IU was administered intrauterine before ET }\end{array}$ \\
\hline & Control $(n=109)$ : no intrauterine hCG was administered \\
\hline Outcomes & Clinical pregnancy, ectopic pregnancy \\
\hline \multirow[t]{3}{*}{ Notes } & Study authors mention "prospective observational study", but the design was in fact RCT. \\
\hline & Location: Reproductive Medicine Centre, PROCREA, Mexico City \\
\hline & Period: August 2011 to November 2012 \\
\hline
\end{tabular}


Santibañez 2014 (Continued)

\section{Power calculation: yes}

Funding: PROCREA

Trial registration: not mentioned and not found

Publication type: full text

\section{Risk of bias}

\begin{tabular}{lll}
\hline Bias & Authors' judgement & Support for judgement \\
\hline $\begin{array}{l}\text { Random sequence genera- } \\
\text { tion (selection bias) }\end{array}$ & Low risk & $\begin{array}{l}\text { A simple randomisation sample and assignment were generated in a comput- } \\
\text { er-based programme. }\end{array}$ \\
\hline $\begin{array}{l}\text { Allocation concealment } \\
\text { (selection bias) }\end{array}$ & Unclear risk & Not mentioned \\
\hline $\begin{array}{l}\text { Blinding of participants } \\
\text { and personnel (perfor- } \\
\text { mance bias) } \\
\text { All outcomes }\end{array}$ & High risk & Not mentioned \\
\hline
\end{tabular}

Blinding of outcome as- Low risk

Not mentioned, but unlikely to induce bias

sessment (detection bias)

All outcomes

Incomplete outcome data $\quad$ Low risk $\quad$ All women followed up until pregnancy test/ultrasound scan
(attrition bias)

All outcomes

Selective reporting (re- Unclear risk No reporting on live birth and miscarriage despite mention of follow-up porting bias)

Other bias Low risk Similar baseline characteristics between groups after randomisation

Singh 2014

Methods Design: 2-arm parallel RCT

Participants

Number: 216

Women's age (mean years; experimental vs control): 35 vs 34.5 (from ESHRE 2014 oral presentation)

Inclusion criteria: infertile women aged < 42 years; recurrent implantation failure

Exclusion criteria: not mentioned

Ovarian controlled hyperstimulation: based on individual participant characteristics (from ESHRE 2014 oral presentation)

\section{Fertilisation: ICSI}

Stage of the embryo at transfer: cleavage

Embryo processing: not mentioned

Number of embryos transferred (mean; experimental vs control): 2.7 vs 2.5 (from ESHRE 2014 oral presentation) 
Singh 2014 (Continued)

Interventions

Experimental ( $\mathbf{n}=\mathbf{1 0 8}$ ): intrauterine administration of rhCG $500 \mathrm{IU}$ in $40 \mu \mathrm{L} 5$ minutes before ET

Control ( $\mathbf{n}=\mathbf{1 0 8})$ : culture medium administered only before ET (from ESHRE 2014 oral presentation)

\begin{tabular}{|c|c|c|}
\hline Outcomes & \multicolumn{2}{|c|}{ Clinical pregnancy, miscarriage, live birth (from ESHRE 2014 oral presentation) } \\
\hline \multirow[t]{6}{*}{ Notes } & \multicolumn{2}{|c|}{ Location: Bhopal Test Tube Baby Centre, Infertility, Bhopal, India } \\
\hline & \multicolumn{2}{|l|}{ Period: 2006 to 2013} \\
\hline & \multicolumn{2}{|c|}{ Power calculation: not mentioned } \\
\hline & \multicolumn{2}{|c|}{ Funding: Bhopal Test Tube Baby Centre } \\
\hline & \multicolumn{2}{|c|}{ Trial registration: $\mathrm{BTTB} / 2006 / 19(?)$} \\
\hline & \multicolumn{2}{|c|}{ Publication type: abstract } \\
\hline \multicolumn{3}{|l|}{ Risk of bias } \\
\hline Bias & Authors' judgement & Support for judgement \\
\hline $\begin{array}{l}\text { Random sequence genera- } \\
\text { tion (selection bias) }\end{array}$ & Low risk & $\begin{array}{l}\text { Participants were randomly divided into } 2 \text { groups via a computer-generated } \\
\text { list. }\end{array}$ \\
\hline $\begin{array}{l}\text { Allocation concealment } \\
\text { (selection bias) }\end{array}$ & Unclear risk & Not mentioned \\
\hline $\begin{array}{l}\text { Blinding of participants } \\
\text { and personnel (perfor- } \\
\text { mance bias) } \\
\text { All outcomes }\end{array}$ & High risk & Not mentioned \\
\hline $\begin{array}{l}\text { Blinding of outcome as- } \\
\text { sessment (detection bias) } \\
\text { All outcomes }\end{array}$ & Low risk & Not mentioned, but unlikely to induce bias \\
\hline $\begin{array}{l}\text { Incomplete outcome data } \\
\text { (attrition bias) } \\
\text { All outcomes }\end{array}$ & Low risk & Zero women lost to follow-up \\
\hline $\begin{array}{l}\text { Selective reporting (re- } \\
\text { porting bias) }\end{array}$ & Low risk & Reported on all important outcomes \\
\hline Other bias & Low risk & Similar baseline characteristics between groups after randomisation \\
\hline
\end{tabular}

Wirleitner 2015a

\begin{tabular}{ll}
\hline Methods & Design: 4-arm parallel RCT (same intervention on day 3 or 5$)$ \\
\hline Participants & Number: $182+1004=1186$ \\
& Women's age (mean years; experimental vs control): 36.1 vs $35.5 ; 37.1$ vs 36.7 \\
& Inclusion criteria: fresh autologous blastocyst transfer on day 5; woman aged $\leq 43$ years \\
& $\begin{array}{l}\text { Exclusion criteria: oocyte donation cycles; women with reported recurrent implantation failure }(\geq 3 \\
\text { negative IVF cycles) }\end{array}$ \\
\hline
\end{tabular}


Wirleitner 2015a (Continued)

Ovarian controlled hyperstimulation: $\mathrm{GnRH}$ agonist long protocol

Fertilisation: IVF or IMSI

Stage of the embryo at transfer: blastocyst

Embryo processing: fresh

Number of embryos transferred: 1 or 2

Interventions

Experimental (day 3) ( $\mathbf{n}=\mathbf{8 9}$ ): intrauterine hCG 500 IU (Pregnyl, Organon, Netherlands) dissolved in 40 $\mu \mathrm{L}$ embryo culture medium G-2 PLUS (Vitrolife, Göteborg, Sweden) administered on day 3 (2 days before ET)

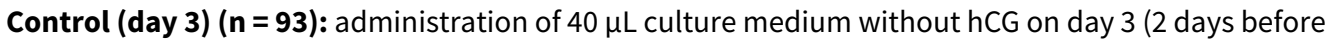
ET)

Experimental (day 5) ( $\mathbf{n}=\mathbf{5 1 0}$ ): intrauterine hCG 500 IU (Pregnyl, Organon, Netherlands) dissolved in $40 \mu \mathrm{L}$ embryo culture medium G-2 PLUS (Vitrolife, Göteborg, Sweden) administered on day 5 ( 3 minutes before ET)

Control (day 5) (n= 494): administration of $40 \mu \mathrm{L}$ culture medium without hCG on day 3 ( 3 minutes before ET)

\begin{tabular}{|c|c|c|}
\hline Outcomes & \multicolumn{2}{|c|}{ Clinical pregnancy, miscarriage, live birth } \\
\hline \multirow[t]{6}{*}{ Notes } & \multicolumn{2}{|c|}{ Location: IVF Centers Prof. Zech, Bregenz, Austria } \\
\hline & \multicolumn{2}{|c|}{ Period: February 2013 to February 2014} \\
\hline & \multicolumn{2}{|c|}{ Power calculation: met only for day 5 administration } \\
\hline & \multicolumn{2}{|c|}{ Funding: not mentioned } \\
\hline & \multicolumn{2}{|c|}{ Trial registration: not mentioned and not found } \\
\hline & \multicolumn{2}{|c|}{ Publication type: full text } \\
\hline \multicolumn{3}{|l|}{ Risk of bias } \\
\hline Bias & Authors' judgement & Support for judgement \\
\hline $\begin{array}{l}\text { Random sequence genera- } \\
\text { tion (selection bias) }\end{array}$ & Low risk & Randomisation was done electronically with a random number generator. \\
\hline $\begin{array}{l}\text { Allocation concealment } \\
\text { (selection bias) }\end{array}$ & Unclear risk & Not mentioned \\
\hline $\begin{array}{l}\text { Blinding of participants } \\
\text { and personnel (perfor- } \\
\text { mance bias) } \\
\text { All outcomes }\end{array}$ & High risk & Participants blinded, but not personnel \\
\hline
\end{tabular}

$\begin{array}{ll}\text { Blinding of outcome as- } & \text { Low risk } \quad \text { Not blinded, but unlikely to induce bias } \\ \text { sessment (detection bias) }\end{array}$

sessment (detection bias)

All outcomes

Incomplete outcome data Unclear risk 19 participants lost to follow-up
(attrition bias)


Wirleitner 2015a (Continued)

Selective reporting (re- Low risk $\quad$ Reports on all relevant outcomes
porting bias)

\begin{tabular}{ll}
\hline Other bias Low risk & $\begin{array}{l}\text { Baseline characteristics of participants were comparable between } 2 \text { study } \\
\text { groups. }\end{array}$
\end{tabular}

Wirleitner 2015b

\begin{tabular}{|c|c|c|}
\hline Methods & \multicolumn{2}{|c|}{ Design: 2-arm parallel RCT } \\
\hline \multirow[t]{9}{*}{ Participants } & \multicolumn{2}{|l|}{ Number: 480} \\
\hline & \multicolumn{2}{|c|}{ Women's age (mean years; experimental vs control): 40.3 vs 40.4} \\
\hline & \multicolumn{2}{|c|}{ Inclusion criteria: women aged 38 to 43 years } \\
\hline & \multicolumn{2}{|c|}{ Exclusion criteria: recurrent implantation failure } \\
\hline & \multicolumn{2}{|c|}{ Ovarian controlled hyperstimulation: $\mathrm{GnRH}$ agonist long protocol } \\
\hline & \multicolumn{2}{|l|}{ Fertilisation: IMSI } \\
\hline & \multicolumn{2}{|c|}{ Stage of the embryo at transfer: blastocyst } \\
\hline & \multicolumn{2}{|c|}{ Embryo processing: fresh } \\
\hline & \multicolumn{2}{|c|}{ Number of embryos transferred: 1 or 2} \\
\hline \multirow[t]{2}{*}{ Interventions } & \multirow{2}{*}{\multicolumn{2}{|c|}{$\begin{array}{l}\text { Experimental }(\mathbf{n}=\mathbf{2 5 5}) \text { : intrauterine } \mathrm{hCG} 500 \mathrm{IU} \text { dissolved in } 40 \mu \mathrm{L} \text { embryo culture medium adminis- } \\
\text { tered } 3 \text { minutes before ET } \\
\text { Control }(\mathbf{n}=\mathbf{2 2 5}) \text { : administration of } 40 \mu \mathrm{L} \text { culture medium without hCG } 3 \text { minutes before ET }\end{array}$}} \\
\hline & & \\
\hline Outcomes & \multicolumn{2}{|c|}{ Clinical pregnancy, miscarriage, live birth } \\
\hline \multirow[t]{6}{*}{ Notes } & \multicolumn{2}{|c|}{ Location: IVF-Centers Prof. Zech, Bregenz, Austria } \\
\hline & \multicolumn{2}{|l|}{ Period: not mentioned } \\
\hline & \multicolumn{2}{|l|}{ Power calculation: yes } \\
\hline & \multicolumn{2}{|c|}{ Funding: funded by hospital/clinic(s) - this study was not externally funded } \\
\hline & \multicolumn{2}{|c|}{ Trial registration: CRT 355} \\
\hline & \multicolumn{2}{|c|}{ Publication type: abstract } \\
\hline \multicolumn{3}{|l|}{ Risk of bias } \\
\hline Bias & Authors' judgement & Support for judgement \\
\hline $\begin{array}{l}\text { Random sequence genera- } \\
\text { tion (selection bias) }\end{array}$ & Unclear risk & Randomisation was mentioned without further details. \\
\hline $\begin{array}{l}\text { Allocation concealment } \\
\text { (selection bias) }\end{array}$ & Unclear risk & Not mentioned \\
\hline
\end{tabular}


Wirleitner 2015b (Continued)
Blinding of participants
Low risk
Participants were blinded. and personnel (performance bias)

All outcomes

\begin{tabular}{lll}
\hline $\begin{array}{l}\text { Blinding of outcome as- } \\
\text { sessment (detection bias) } \\
\text { All outcomes }\end{array}$ & Low risk & Not blinded, but unlikely to induce bias \\
\hline $\begin{array}{l}\text { Incomplete outcome data } \\
\begin{array}{l}\text { (attrition bias) } \\
\text { All outcomes }\end{array}\end{array}$ & Low risk & All participants were followed up. \\
\hline $\begin{array}{l}\text { Selective reporting (re- } \\
\text { porting bias) }\end{array}$ & Low risk & Reports on all relevant outcomes \\
\hline Other bias & Low risk & $\begin{array}{l}\text { Baseline characteristics of participants were comparable between } 2 \text { study } \\
\text { groups. }\end{array}$ \\
\hline
\end{tabular}

Zarei 2014

Methods Design: 2-arm parallel RCT

Participants

Number: 210

Women's age (mean years; experimental vs control): 29.9 vs 31.2

Inclusion criteria: 18 - to 40 -year-old women with infertility

Exclusion criteria: women with autoimmune disorders, endocrinopathies, who had previous successful IVF/ICSI trials; endometriosis; azoospermia; hydrosalpinges

Ovarian controlled hyperstimulation: not mentioned

Fertilisation: ICSI

Stage of the embryo at transfer: cleavage

Embryo processing: not mentioned (likely fresh)

Number of embryos transferred (mean; experimental vs control): 6.1 vs 5.7

Interventions Experimental ( $\mathbf{n}=\mathbf{1 0 5})$ : $r h C G 250 \mu \mathrm{g}(0.5 \mathrm{~mL}, 6500 \mathrm{IU})$ (Ovitrelle, Merck Serono, France) through intrauterine injection 12 minutes before ET

Control ( $\mathbf{n}=\mathbf{1 0 5})$ : intrauterine injection of normal saline $(0.5 \mathrm{~mL}) 12$ minutes before ET

Outcomes Clinical pregnancy, miscarriage, ectopic pregnancy, stillbirth

Notes

Location: Reproductive Medicine Center of Mother and Child Hospital, Shiraz, Iran

Period: December 2011 to November 2012

Power calculation: yes

Funding: Shiraz University of Medical Sciences

Trial registration: IRCT2012121711790N1 
Zarei 2014 (Continued)

Publication type: full text

\section{Risk of bias}

\begin{tabular}{|c|c|c|}
\hline Bias & Authors' judgement & Support for judgement \\
\hline $\begin{array}{l}\text { Random sequence genera- } \\
\text { tion (selection bias) }\end{array}$ & Low risk & $\begin{array}{l}\text { Participants were randomly assigned to } 2 \text { study groups via a computerised } \\
\text { random digit generator based on their registration number in order of referral. }\end{array}$ \\
\hline $\begin{array}{l}\text { Allocation concealment } \\
\text { (selection bias) }\end{array}$ & Unclear risk & Not mentioned \\
\hline $\begin{array}{l}\text { Blinding of participants } \\
\text { and personnel (perfor- } \\
\text { mance bias) } \\
\text { All outcomes }\end{array}$ & Low risk & $\begin{array}{l}\text { The syringes with volume of } 0.5 \mathrm{~mL} \text { from each group were prepared by the fel- } \\
\text { lowship student and injected blinded by the attending gynaecologist. }\end{array}$ \\
\hline $\begin{array}{l}\text { Blinding of outcome as- } \\
\text { sessment (detection bias) } \\
\text { All outcomes }\end{array}$ & Low risk & $\begin{array}{l}\text { Double-blinding mentioned (? women ? outcome assessors - in addition to gy- } \\
\text { naecologists performing the transfer), unlikely to induce bias }\end{array}$ \\
\hline $\begin{array}{l}\text { Incomplete outcome data } \\
\text { (attrition bias) } \\
\text { All outcomes }\end{array}$ & High risk & $\begin{array}{l}\text { 23/105 participants in intrauterine rhCG group and } 7 / 105 \text { participants in place- } \\
\text { bo group were lost to follow-up after receiving the allocated treatment (un- } \\
\text { clear why). }\end{array}$ \\
\hline $\begin{array}{l}\text { Selective reporting (re- } \\
\text { porting bias) }\end{array}$ & Unclear risk & No report on live births \\
\hline Other bias & Low risk & $\begin{array}{l}\text { Baseline characteristics of participants were comparable between } 2 \text { study } \\
\text { groups. }\end{array}$ \\
\hline
\end{tabular}

ART: assisted reproductive technology; BMI: body mass index; ET: embryo transfer; ESHRE: European Society of Human Reproduction and Embryology; FET: frozen embryo transfer; FSH: follicle-stimulating hormone; GnRH: gonadotropin-releasing hormone; hCG: human chorionic gonadotropin; ICSI: intracytoplasmic sperm injection; IMSI: intracytoplasmic morphologically selected sperm injection; IU: international unit; IVF: in vitro fertilisation; PCOS: polycystic ovary syndrome; RCT: randomised controlled trial; rhCG: recombinant human chorionic gonadotropin.

Characteristics of excluded studies [ordered by study ID]

\begin{tabular}{ll}
\hline Study & Reason for exclusion \\
\hline Dieamant 2016 & Meta-analysis \\
\hline Giuliani 2015 & Participants were oocyte donors who did not undergo embryo transfer \\
\hline Huang 2017 & Retrospective \\
\hline Janati 2013 & $\begin{array}{l}\text { Included in the first review; replaced now by more recent full publication (Dehghani Firouzabadi } \\
\text { 2016) }\end{array}$ \\
\hline Jeong 2013 & Retrospective \\
\hline Kanter 2017 & Retrospective \\
\hline Li 2013 & Not randomised \\
\hline
\end{tabular}




\begin{tabular}{ll}
\hline Study & Reason for exclusion \\
\hline Osman 2016 & Meta-analysis \\
\hline Rebolloso 2013 & Not randomised \\
\hline Riboldi 2013 & Not randomised \\
\hline Strug 2016 & Participants were oocyte donors who did not undergo embryo transfer. \\
\hline Volovsky 2016 & Case control \\
\hline Ye 2015 & Meta-analysis \\
\hline
\end{tabular}

Characteristics of studies awaiting assessment [ordered by study ID]

Badehnoosh 2014

\begin{tabular}{|c|c|}
\hline Methods & Design: 2-arm parallel RCT \\
\hline \multirow[t]{9}{*}{ Participants } & Number: 80 \\
\hline & Women's age (mean years; experimental vs control): 29.5 vs 29.3 \\
\hline & Inclusion criteria: women undergoing ICSI \\
\hline & Exclusion criteria: not mentioned \\
\hline & Ovarian controlled hyperstimulation: not mentioned \\
\hline & Fertilisation: ICSI \\
\hline & Stage of the embryo at transfer: not mentioned \\
\hline & Embryo processing: not mentioned \\
\hline & Number of embryos transferred (mean; experimental vs control): 2.9 vs 2.8 \\
\hline \multirow[t]{2}{*}{ Interventions } & $\begin{array}{l}\text { Experimental: intrauterine injection of hCG } 500 \mathrm{IU} \text { dissolved in } 40 \mu \mathrm{L} \text { of ET media } 10 \text { minutes be- } \\
\text { fore ET }\end{array}$ \\
\hline & Control: $40 \mu \mathrm{L}$ of ET media 10 minutes before ET \\
\hline Outcomes & Implantation rate defined as positive pregnancy test at 2 weeks after ET (biochemical pregnancy) \\
\hline \multirow[t]{7}{*}{ Notes } & $\begin{array}{l}\text { We emailed the study authors in February } 2016 \text { and January } 2018 \text { for more information on study } \\
\text { design and outcomes. No reply has yet been received. }\end{array}$ \\
\hline & Location: Avicenna Infertility Clinic, Tehran, Iran \\
\hline & Period: not mentioned \\
\hline & Power calculation: not mentioned \\
\hline & Funding: not mentioned \\
\hline & Trial registration: not mentioned and not found \\
\hline & Publication type: abstract \\
\hline
\end{tabular}


Bhat 2014

\begin{tabular}{|c|c|}
\hline Methods & Design: 2-arm parallel RCT \\
\hline \multirow[t]{9}{*}{ Participants } & Number: 32 \\
\hline & Women's age (mean years; experimental vs control): 29.6 vs 29.6 \\
\hline & Inclusion criteria: women undergoing IVF \\
\hline & Exclusion criteria: not mentioned \\
\hline & Ovarian controlled hyperstimulation: not mentioned \\
\hline & Fertilisation: IVF or ICSI \\
\hline & Stage of the embryo at transfer: cleavage \\
\hline & Embryo processing: fresh and frozen/thawed \\
\hline & Number of embryos transferred (mean; experimental vs control): 2.9 vs 2.9 \\
\hline \multirow[t]{2}{*}{ Interventions } & Experimental: intrauterine administration of hCG 500 IU 7 minutes before ET \\
\hline & Control: ET without hCG \\
\hline Outcomes & Fertilisation rate \\
\hline \multirow[t]{7}{*}{ Notes } & $\begin{array}{l}\text { We emailed the study authors in February } 2016 \text { and January } 2018 \text { for more information on study } \\
\text { design and outcomes. No reply has yet been received. }\end{array}$ \\
\hline & Location: Radhakrishna Multispeciality Hospital and IVF Centre in Bengaluru in Southern India \\
\hline & Period: April 2013 to March 2014 \\
\hline & Power calculation: not mentioned \\
\hline & Funding: none \\
\hline & Trial registration: not mentioned and not found \\
\hline & Publication type: full text \\
\hline
\end{tabular}

ET: embryo transfer; hCG: human chorionic gonadotropin; ICSI: intracytoplasmic sperm injection; IU: international unit; IVF: in vitro fertilisation; RCT: randomised controlled trial.

Characteristics of ongoing studies [ordered by study ID]

\section{IRCT2017041733486N1}

Trial name or title
Evaluation effect of intrauterine injection of human chorionic gonadotropin before embryo transfer on implantation rate and pregnancy rate in frozen cycles on IVF/ICSI

Randomised controlled trial

\begin{tabular}{ll}
\hline Participants & Women undergoing embryo transfer \\
\hline Interventions & $\begin{array}{l}\text { Experimental: interventional group ( } \mathrm{n} 130) \text { was injected with } 500 \text { IU of intrauterine hCG before } \\
\text { embryo transfer } \\
\text { Control: the second group }(n=130) \text { did not receive administration of } 500 \text { IU hCG }\end{array}$ \\
\hline
\end{tabular}

Intrauterine administration of human chorionic gonadotropin (hCG) for subfertile women undergoing assisted reproduction (Review) 
IRCT2017041733486N1 (Continued)

Outcomes Chemical and clinical pregnancy, implantation, miscarriage, ectopic pregnancy

\begin{tabular}{ll}
\hline Starting date & October 2017 \\
\hline Contact information & Ziaee Zohreh; 00982188896692; ziaee-z@razi.tums.ac.ir
\end{tabular}

Notes

\section{NCT02668965}

\begin{tabular}{ll}
\hline Trial name or title & The effects of intrauterine infusion of hCG at the time of embryo transfer \\
\hline Methods & Randomised controlled trial \\
\hline Participants & Women undergoing embryo transfer
\end{tabular}

Interventions Experimental: intrauterine infusion with hCG (500 IU) 10 microliters before embryo transfer

Control: intrauterine infusion with standard embryo culture media 10 microliters before embryo transfer

Outcomes Implantation, chemical and clinical pregnancy

\begin{tabular}{ll}
\hline Starting date & December 2015 \\
\hline Contact information & Savinee Boonsuk, MD; +66818706643; noomnim_mu@hotmail.com \\
\hline
\end{tabular}

\section{Notes}

\section{NCT02825108}

\begin{tabular}{ll}
\hline Trial name or title & $\begin{array}{l}\text { Intrauterine injection of human chorionic gonadotropin injection (hCG) before embryo transfer on } \\
\text { pregnancy outcomes in frozen embryo transfer cycles }\end{array}$
\end{tabular}

\begin{tabular}{ll}
\hline Methods & $\begin{array}{l}\text { Randomised double-blind clinical trial to evaluate the effect of intrauterine injection of human } \\
\text { chorionic gonadotropin ( } \mathrm{hCG} \text { ) before frozen embryo transfer (ET) }\end{array}$
\end{tabular}

\begin{tabular}{ll}
\hline Participants & All patients with primary infertility who have only 1 fresh implantation failure and are undergoing \\
frozen embryo transfer cycles were enrolled.
\end{tabular}
frozen embryo transfer cycles were enrolled.

\begin{tabular}{ll}
\hline Interventions & Experimental: participants receive $40 \mu \mathrm{L}$ of tissue culture medium (G.2plus ref. 10132, Vitrolife, \\
& Göteborg, Sweden) containing 500 IU of hCG (Choriomon, IBSA SA, Switzerland), which is injected \\
& intrauterine, approximately 7 minutes before embryo transfer
\end{tabular}

Control: patients receive only $40 \mu \mathrm{L}$ of tissue culture medium (G.2plus ref. 10132, Vitrolife, Göteborg, Sweden), which is injected intrauterine, approximately 7 minutes before embryo transfer

\begin{tabular}{ll}
\hline Outcomes & Implantation, pregnancy loss/miscarriage \\
\hline Starting date & January 2015 \\
\hline Contact information & $\begin{array}{l}\text { Nasser Aghdami, MD, PhD; (+98)23562000 ext 516; nasser.aghdami@royaninstitute.org } \\
\text { Leila Arab, MD; (+98)23562000 ext 414; leara91@gmail.com }\end{array}$ \\
\hline \hline
\end{tabular}


NCT02825108 (Continued)
Notes
Contact: Leila Arab, MD

\section{NCT02870855}

\begin{tabular}{ll}
\hline Trial name or title & $\begin{array}{l}\text { Effect of intrauterine injection of hCG on pregnancy outcome in repeated implantation failure pa- } \\
\text { tients }\end{array}$ \\
\hline Methods & Randomised controlled trial \\
\hline Participants & Women who undergo frozen ET \\
\hline Interventions & Control: no hCG injection \\
\hline Outcomes & Clinical pregnancy, miscarriage, ectopic pregnancy \\
\hline Starting date & July 2017 \\
\hline Contact information & Yuan Li, doctor; +86-731-82355100; 1002251255@qq.com \\
\hline Notes & \\
\hline
\end{tabular}

\section{NCT03238807}

\begin{tabular}{ll}
\hline Trial name or title & Effect of intrauterine injection of hCG before ET on clinical outcomes in IVF/ICSI cycles \\
\hline Methods & $\begin{array}{l}\text { Randomised controlled trial (RCT) to detect whether intrauterine injection of hCG before ET im- } \\
\text { proves clinical outcomes in IVF/ICSI cycles }\end{array}$
\end{tabular}

Participants Women undergoing IVF/ICSI

Interventions Experimental: $0.1 \mathrm{~mL}$ of the tissue culture medium with 500 IU hCG will be injected inside the
uterus before ET

Control: $0.1 \mathrm{~mL}$ of the tissue culture medium without hCG will be injected inside the uterus before ET

\begin{tabular}{ll}
\hline Outcomes & Implantation, clinical pregnancy, miscarriage, live birth \\
\hline Starting date & October 2017 \\
\hline Contact information & KArim S Abdallah, MSc; +201000024188; karimsayed88@hotmail.com \\
\hline Notes & \\
\hline
\end{tabular}

ET: embryo transfer; hCG: human chorionic gonadotropin; ICSI: intracytoplasmic sperm injection; IVF: in vitro fertilisation; RCT: randomised controlled trial. 
DATA AND ANALYSES

Comparison 1. Intrauterine human chorionic gonadotropin (hCG) versus no hCG

\begin{tabular}{|c|c|c|c|c|}
\hline Outcome or subgroup title & No. of studies & $\begin{array}{l}\text { No. of partici- } \\
\text { pants }\end{array}$ & Statistical method & Effect size \\
\hline 1 Live birth & 5 & & Risk Ratio (M-H, Random, 95\% Cl) & Subtotals only \\
\hline $\begin{array}{l}\text { 1.1 Cleavage stage: } \mathrm{hCG}<500 \\
\text { IU }\end{array}$ & 1 & 280 & Risk Ratio (M-H, Random, 95\% Cl) & $0.76[0.58,1.01]$ \\
\hline $\begin{array}{l}\text { 1.2 Cleavage stage: } \mathrm{hCG} \geq 500 \\
\text { IU }\end{array}$ & 3 & 914 & Risk Ratio (M-H, Random, 95\% Cl) & $1.57[1.32,1.87]$ \\
\hline $\begin{array}{l}1.3 \text { Blastocyst stage: } \mathrm{hCG} \geq \\
500 \mathrm{IU}\end{array}$ & 2 & 1666 & Risk Ratio (M-H, Random, 95\% Cl) & $0.92[0.80,1.04]$ \\
\hline 2 Miscarriage & 11 & 3927 & Risk Ratio (M-H, Random, 95\% Cl) & $1.04[0.81,1.35]$ \\
\hline $\begin{array}{l}3 \text { Miscarriage per clinical } \\
\text { pregnancy }\end{array}$ & 11 & 1620 & Risk Ratio (M-H, Random, 95\% Cl) & $0.84[0.62,1.13]$ \\
\hline 4 Clinical pregnancy & 16 & & Risk Ratio (M-H, Random, 95\% Cl) & Subtotals only \\
\hline $\begin{array}{l}\text { 4.1 Cleavage stage: } h C G<500 \\
\text { IU }\end{array}$ & 1 & 280 & Risk Ratio (M-H, Random, 95\% Cl) & $0.88[0.70,1.10]$ \\
\hline $\begin{array}{l}\text { 4.2 Cleavage stage: } h C G \geq 500 \\
\text { IU }\end{array}$ & 12 & 2186 & Risk Ratio (M-H, Random, 95\% Cl) & $1.49[1.32,1.68]$ \\
\hline $\begin{array}{l}4.3 \text { Blastocyst stage: } \mathrm{hCG} \geq \\
500 \mathrm{IU}\end{array}$ & 4 & 2091 & Risk Ratio (M-H, Random, 95\% Cl) & $0.99[0.85,1.15]$ \\
\hline 5 Complications & 6 & & $\begin{array}{l}\text { Peto Odds Ratio (Peto, Fixed, 95\% } \\
\mathrm{Cl} \text { ) }\end{array}$ & Subtotals only \\
\hline 5.1 Intrauterine death & 3 & 1078 & $\begin{array}{l}\text { Peto Odds Ratio (Peto, Fixed, 95\% } \\
\mathrm{Cl} \text { ) }\end{array}$ & $0.74[0.31,1.73]$ \\
\hline $\begin{array}{l}5.2 \text { Ectopic or heterotopic } \\
\text { pregnancy }\end{array}$ & 4 & 1073 & $\begin{array}{l}\text { Peto Odds Ratio (Peto, Fixed, 95\% } \\
\mathrm{Cl} \text { ) }\end{array}$ & $0.22[0.04,1.30]$ \\
\hline 5.3 Triplet pregnancy & 1 & 483 & $\begin{array}{l}\text { Peto Odds Ratio (Peto, Fixed, 95\% } \\
\mathrm{Cl} \text { ) }\end{array}$ & $7.55[0.78,72.88]$ \\
\hline
\end{tabular}

Analysis 1.1. Comparison 1 Intrauterine human chorionic gonadotropin (hCG) versus no hCG, Outcome 1 Live birth.

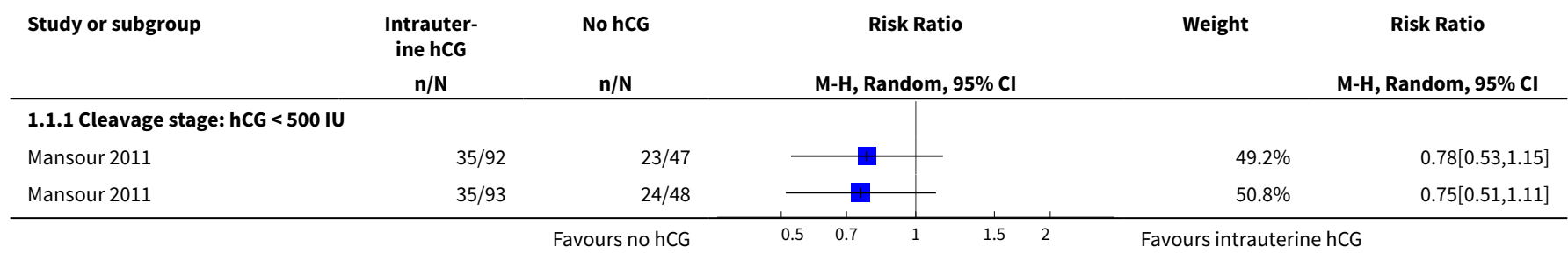




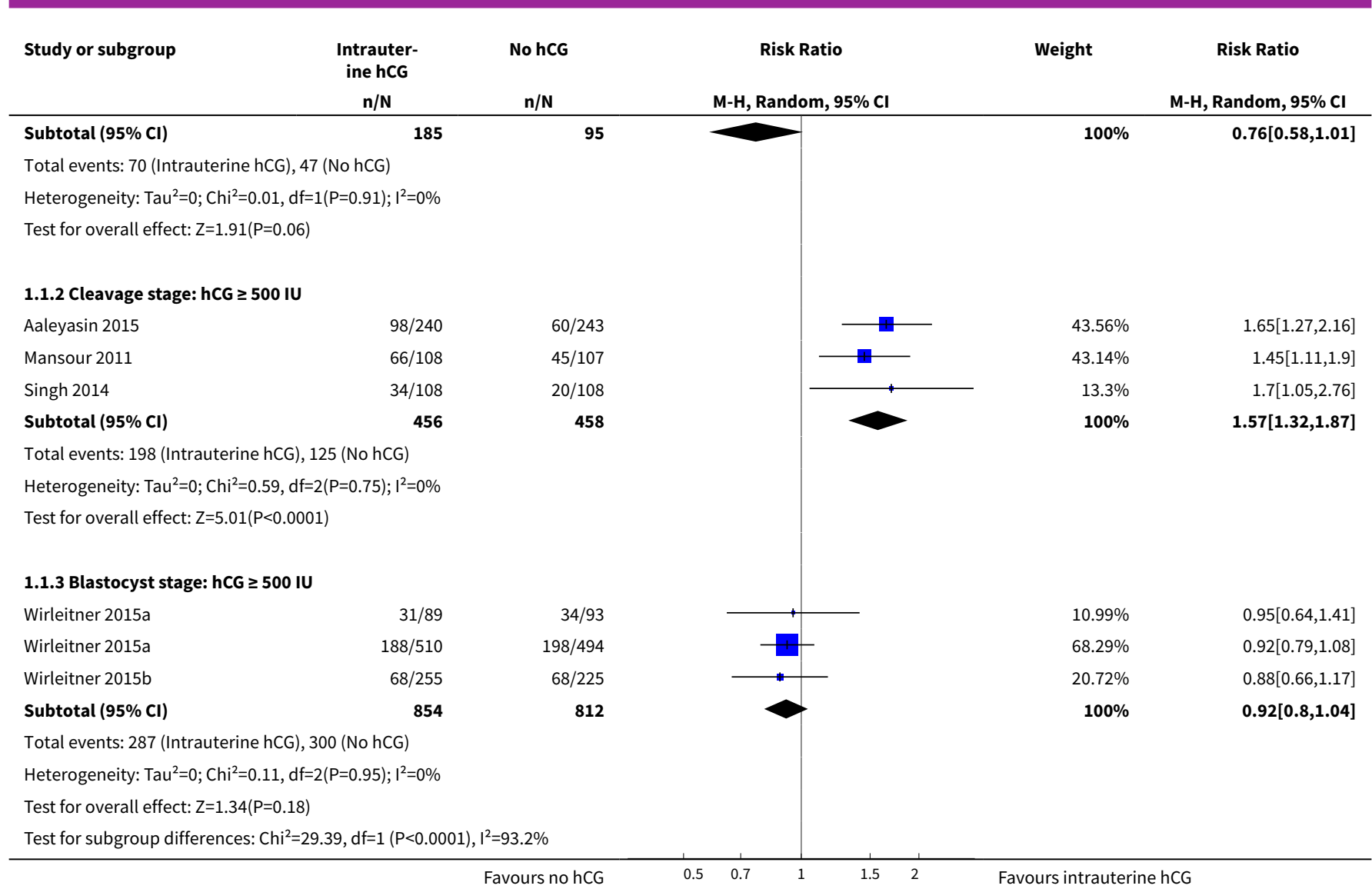

Analysis 1.2. Comparison 1 Intrauterine human chorionic gonadotropin (hCG) versus no hCG, Outcome 2 Miscarriage.

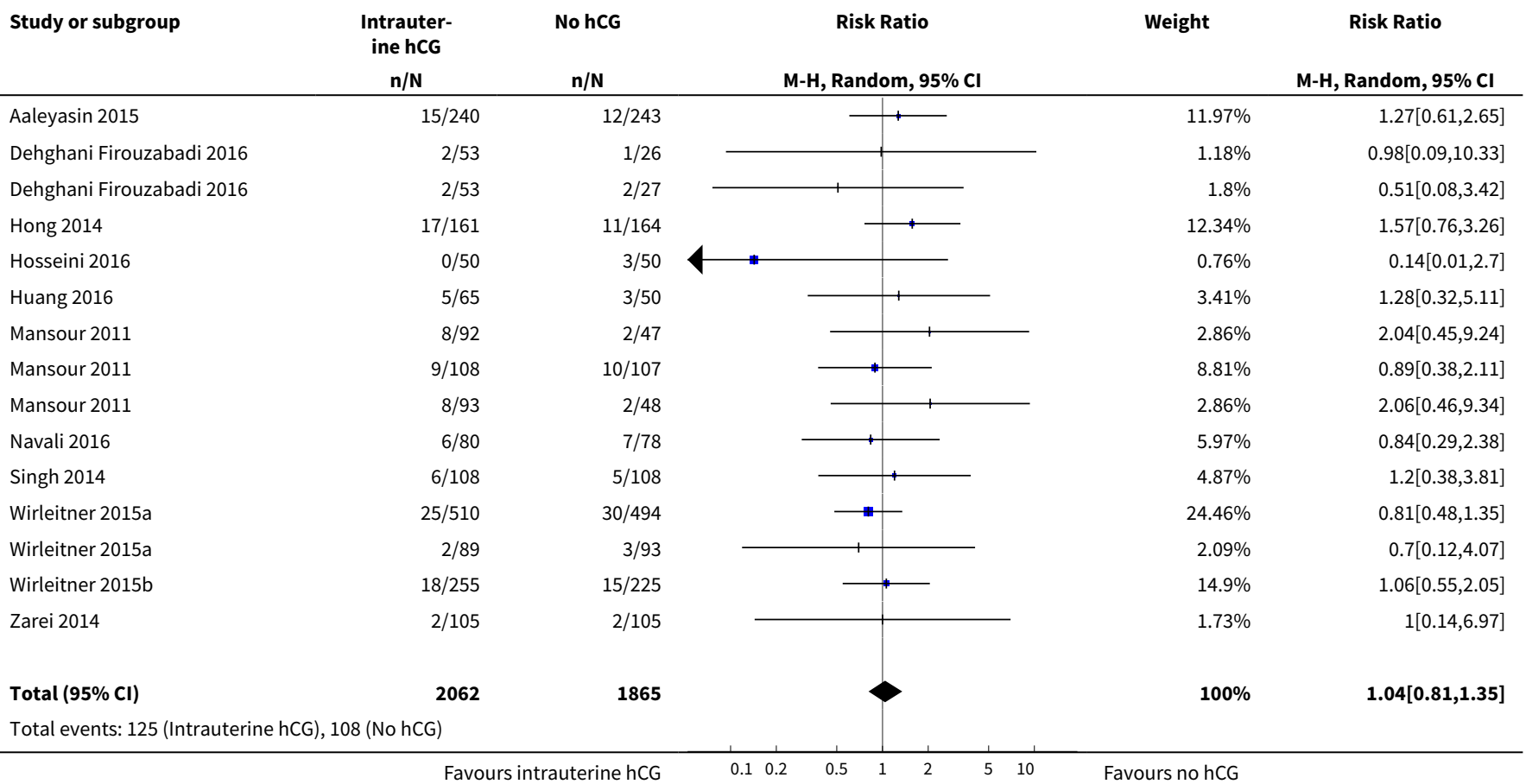




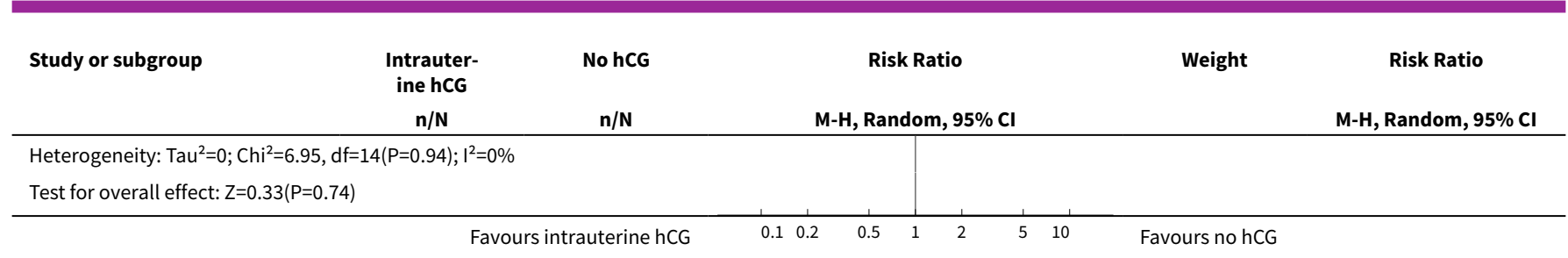

\section{Analysis 1.3. Comparison 1 Intrauterine human chorionic gonadotropin (hCG) versus no hCG, Outcome 3 Miscarriage per clinical pregnancy.}

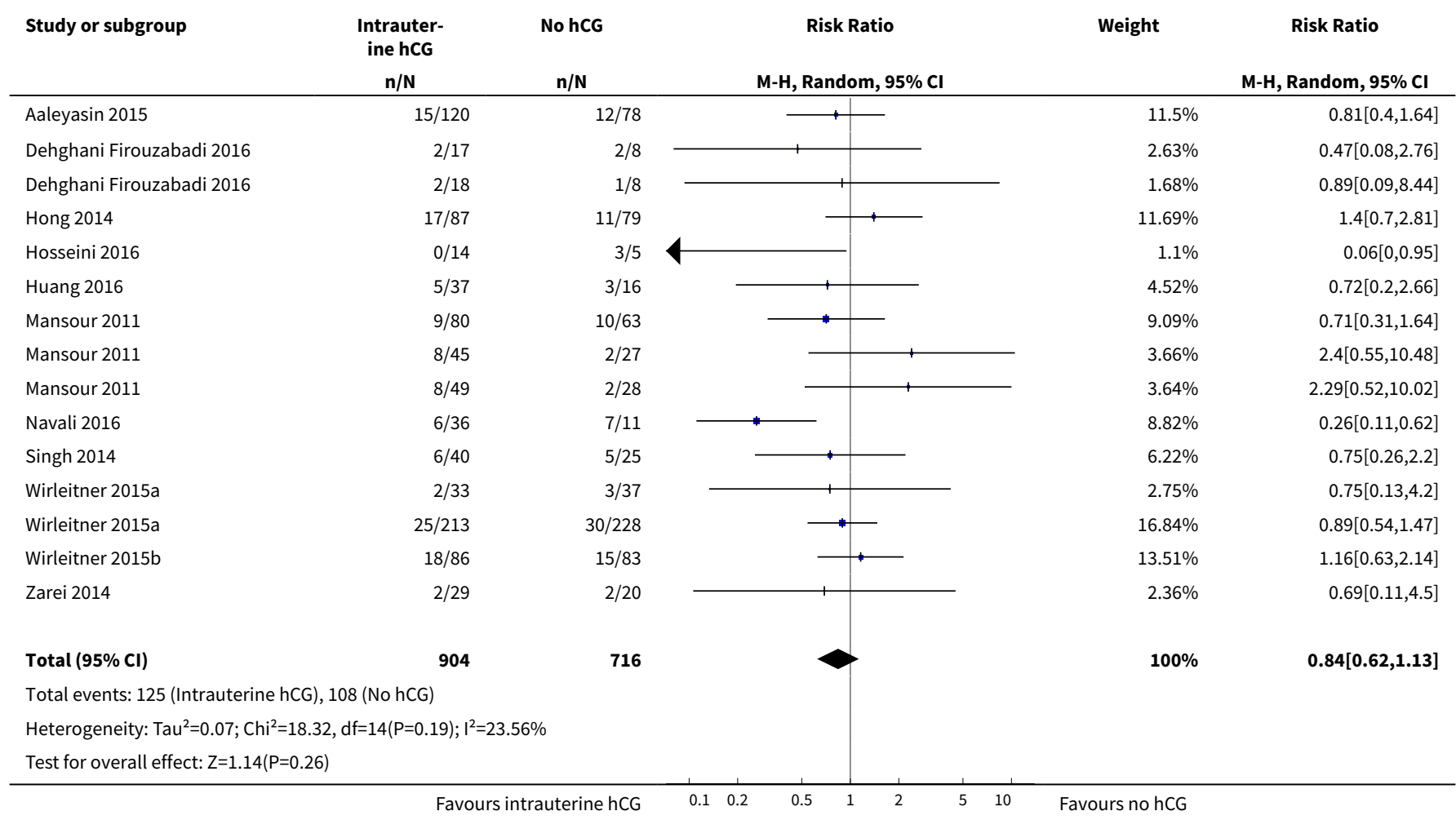

Analysis 1.4. Comparison 1 Intrauterine human chorionic gonadotropin (hCG) versus no hCG, Outcome 4 Clinical pregnancy.

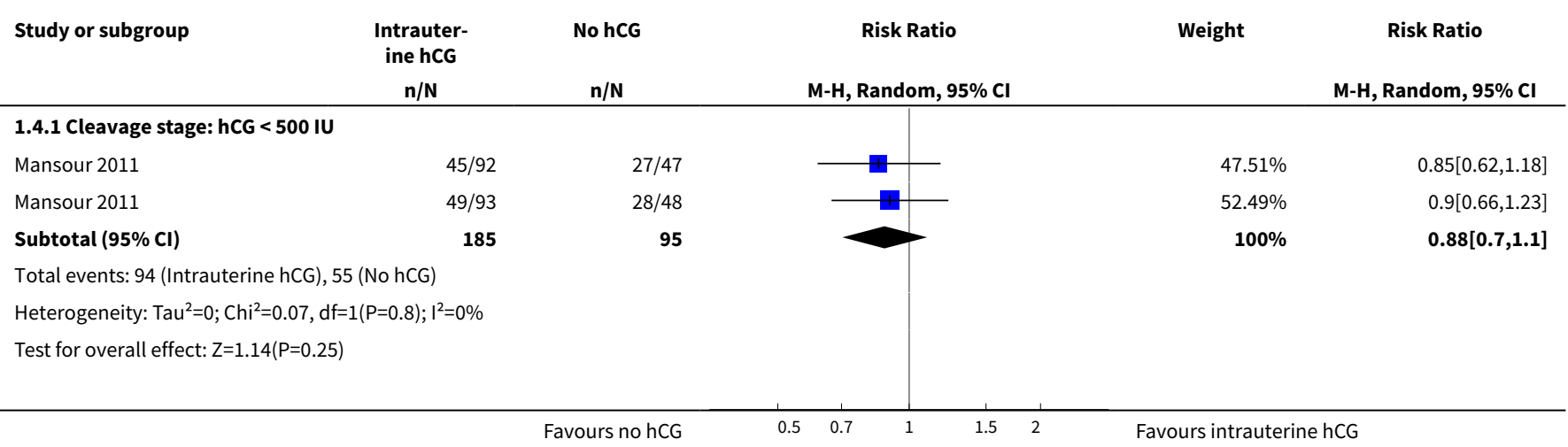




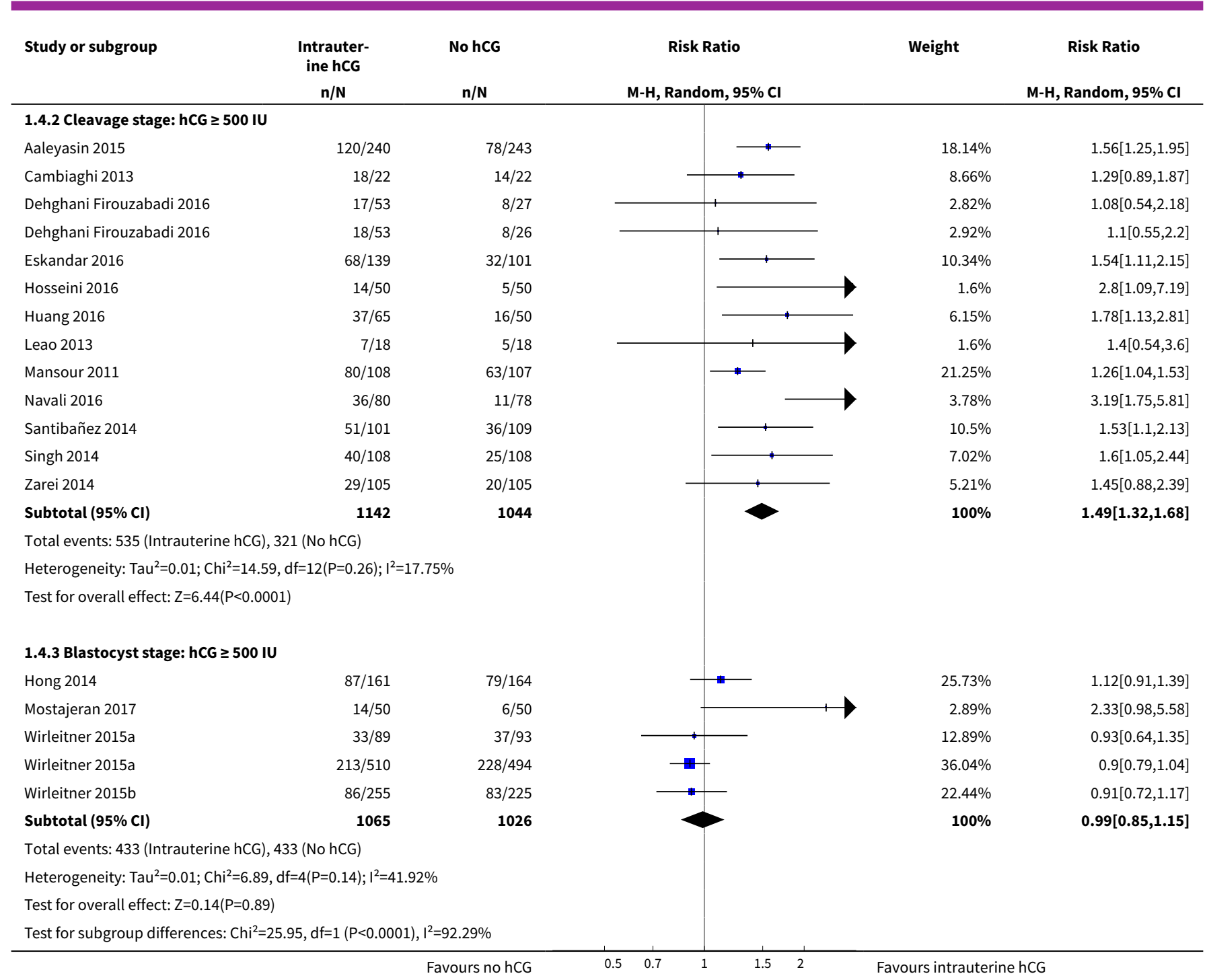

Analysis 1.5. Comparison 1 Intrauterine human chorionic gonadotropin (hCG) versus no hCG, Outcome 5 Complications.

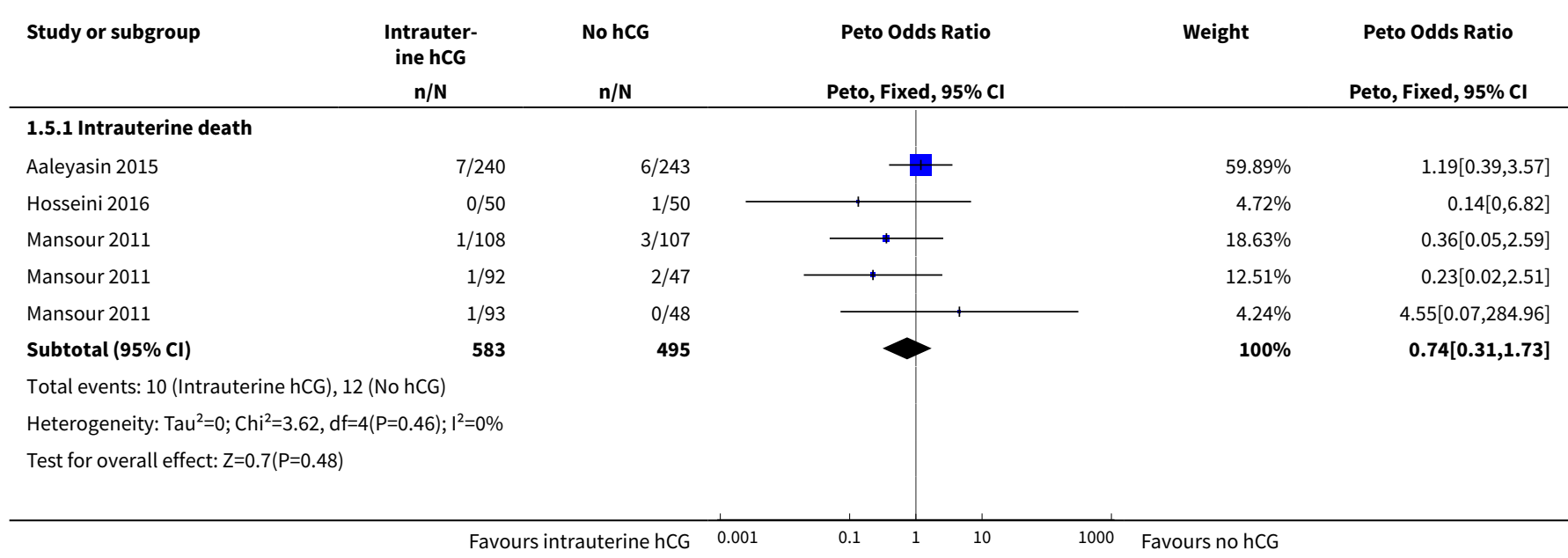




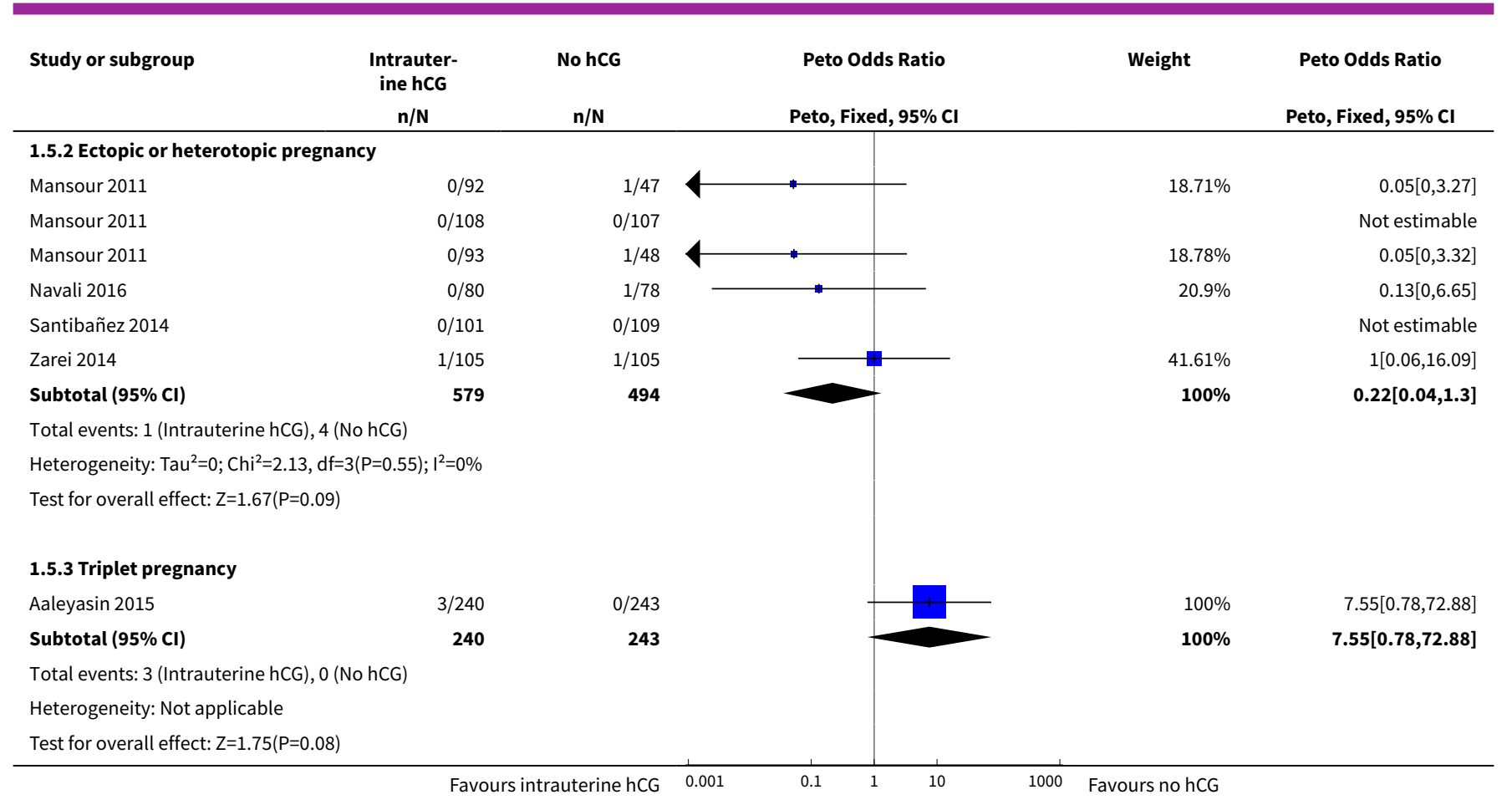

\section{APPENDICES}

\section{Appendix 1. Cochrane Gynaecology and Fertility Group (CGF) Specialised Register search strategy}

\section{PROCITE Platform}

Searched 9 January 2018

Keywords CONTAINS "IVF" or "in vitro fertilization" or "in-vitro fertilisation" or "ICSI" or "intracytoplasmic sperm injection" or "ET" or "Embryo" or "in-vitro fertilization" or "Embryo Transfer" or "Embryo Transfer-uterine" or "blastocyst transfer" or Title CONTAINS "IVF" or "in vitro fertilization" or "in-vitro fertilisation" or "ICSI" or "intracytoplasmic sperm injection" or "Embryo" or "in-vitro fertilization" or "ET" or "Embryo" or "in-vitro fertilization" or "Embryo Transfer" or "Embryo Transfer-uterine" or "blastocyst transfer"

AND

Keywords CONTAINS "HCG " or "human chorionic gonadotrophin" or "human chorionic gonadotropin" or "recombinant HCG" or "rhCG" or Title CONTAINS "HCG " or "human chorionic gonadotrophin" or "human chorionic gonadotropin" or "recombinant HCG" or "rhCG"

AND

Keywords CONTAINS "intrauterine human chorionic gonadotrophin" or "intrauterine" or "Intrauterine injection" or "intrauterine instillation "or "uterine cavity injection" or "endometrial" or "Endometrium" or "uterine" or Title CONTAINS "intrauterine human chorionic gonadotrophin" or "intrauterine" or "Intrauterine injection" or "intrauterine instillation "or "uterine cavity injection" or "Endometrium" or "uterine" (113)

\section{Appendix 2. CENTRAL search strategy}

Web Platform via CENTRAL Register of Studies online (CRSO)

Searched 9 January 2018

\#1 MESH DESCRIPTOR Reproductive Techniques, Assisted EXPLODE ALL TREES 2881

\#2 ( embryo* adj2 transfer*):TI,AB,KY 2522

\#3 (blastocyst* adj2 transfer $\left.{ }^{\star}\right): T I, A B, K Y 264$

Intrauterine administration of human chorionic gonadotropin (hCG) for subfertile women undergoing assisted reproduction (Review) 
\#4 (assisted reproduct* ):TI,AB, KY 851

\#5 (ivf or icsi):TI,AB, KY 4126

\#6 (in vitro fertili?ation):TI,AB,KY 2200

\#7 (intracytoplasmic sperm injection*):TI,AB,KY 1350

\#8 \#1 OR \#2 OR \#3 OR \#4 OR \#5 OR \#6 OR \#7 6556

\#9 MESH DESCRIPTOR Chorionic Gonadotropin EXPLODE ALL TREES 697

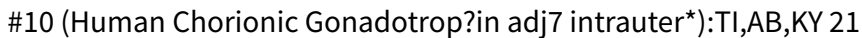

\#11 (Human Chorionic Gonadotrop?in adj7 uter*):TI,AB,KY 6

\#12 ((endometri* adj2 infusion*) and chorionic):TI,AB,KY 3

\#13 ((intra?uter ${ }^{\star}$ adj2 infusion $\left.{ }^{\star}\right)$ and chorionic):TI,AB, KY 6

\#14 ((intra?uter ${ }^{\star}$ adj2 instillation) and chorionic):TI,AB,KY 3

\#15 ((endometri adj2 injection*) and chorionic):TI,AB,KY 3

\#16 ((intra?uter* adj2 injection ${ }^{\star}$ ) and chorionic):TI,AB,KY 36

\#17 ((intra?uter* adj2 administration) and chorionic):TI,AB,KY 28

\#18 (intrauter* adj7 ?hcg):TI,AB,KY 50

\#19 \#9 OR \#10 OR \#11 OR \#12 OR \#13 OR \#14 OR \#15 OR \#16 OR \#17 OR \#18 767

\#20 \#8 AND \#19 493

\section{Appendix 3. MEDLINE search strategy}

OVID Platform

Searched from 1946 to 9 January 2018

1 exp embryo transfer/ or exp fertilization in vitro/ or exp sperm injections, intracytoplasmic/ (40939)

2 embryo transfer\$.tw. (11209)

3 in vitro fertili?ation.tw. (22663)

4 assisted reproduct ${ }^{\star}$.tw. (13334)

5 (ivf or et).tw. (260179)

6 icsi.tw. (7528)

7 intracytoplasmic sperm injection\$.tw. (6590)

8 (blastocyst adj2 transfer\$).tw. (843)

9 or/1-8 (297500)

10 exp Chorionic Gonadotropin/ad, tu, th [Administration \& Dosage, Therapeutic Use, Therapy] (5410)

11 (Human Chorionic Gonadotrop?in adj7 intrauter\$).tw. (87)

12 (Human Chorionic Gonadotrop?in adj7 uter\$).tw. (159)

13 (Human Chorionic Gonadotrop?in adj7 intra-uter\$).tw. (0)

14 ((endometri\$ adj2 infusion\$) and chorionic).tw. (1)

15 ((endometri\$ adj2 ?instillation) and chorionic).tw. (0)

16 ((intra?uter\$ adj2 infusion\$) and chorionic).tw. (6)

17 ((intra?uter\$ adj2 ?instillation) and chorionic).tw. (6)

18 ((endometri\$ adj2 injection\$) and chorionic).tw. (5)

19 ((intra?uter\$ adj2 injection\$) and chorionic).tw. (16)

20 ((intra?uter\$ adj2 administration) and chorionic).tw. (14)

21 ((endometri\$ adj2 administration) and chorionic).tw. (7)

22 (intrauter\$ adj7 ?hcg).tw. (198)

23 (intra-uter\$ adj7 ?hcg).tw. (15)

24 (uter\$ adj7 ?hcg).tw. (342)

25 or/10-24 (6018)

269 and 25 (1776) 
27 randomized controlled trial.pt. (515870)

28 controlled clinical trial.pt. (101741)

29 randomized.ab. (452787)

30 randomised.ab. (91845)

31 placebo.tw. (215895)

32 clinical trials as topic.sh. (202549)

33 randomly.ab. (311971)

34 trial.ti. (203432)

35 (crossover or cross-over or cross over).tw. (83358)

36 or/27-35 (1322190)

37 exp animals/ not humans.sh. (4813914)

3836 not 37 (1219196)

3926 and 38 (369)

\section{Appendix 4. Embase search strategy}

OVID Platform

Searched from 1980 to 9 January 2018

1 exp embryo transfer/ or exp fertilization in vitro/ or exp intracytoplasmic sperm injection/ (58311)

2 embryo $\$$ transfer\$.tw. (17900)

3 in vitro fertili?ation.tw. (26272)

4 assisted reproduct ${ }^{\star}$.tw. (18775)

5 icsi.tw. (13770)

6 intracytoplasmic sperm injection\$.tw. (8240)

7 (blastocyst adj2 transfer\$).tw. (1906)

8 (ivf or et).tw. (606759)

9 or/1-8 (659662)

10 (Human Chorionic Gonadotrop?in adj7 intrauter\$).tw. (122)

11 (Human Chorionic Gonadotrop?in adj7 uter\$).tw. (149)

12 (intrauter\$ adj7 ?hcg).tw. (286)

13 chorionic gonadotropin/dt, ut [Drug Therapy, Intrauterine Drug Administration] (4766)

14 (uter\$ adj3 ?hcg).tw. (127)

15 ((endometri\$ adj2 infusion\$) and chorionic).tw. (2)

16 ((endometri\$ adj2 ?instillation) and chorionic).tw. (0)

17 ((intra?uter\$ adj2 infusion\$) and chorionic).tw. (8)

18 ((intra?uter\$ adj2 ?instillation) and chorionic).tw. (7)

19 ((endometri\$ adj2 injection\$) and chorionic).tw. (5)

20 ((intra?uter\$ adj2 injection\$) and chorionic).tw. (44)

21 ((intra?uter\$ adj2 administration) and chorionic).tw. (30)

22 ((endometri\$ adj2 administration) and chorionic).tw. (14)

23 or/10-22 (5333)

249 and 23 (2692)

25 Clinical Trial/ (962568)

26 Randomized Controlled Trial/ (479015)

27 exp randomization/ (76661)

28 Single Blind Procedure/ (30048)

29 Double Blind Procedure/ (142111)

30 Crossover Procedure/ (53667)

31 Placebo/ (302487)

32 Randomi?ed controlled trial\$.tw. (169852)

33 Rct.tw. (26427)

34 random allocation.tw. (1709)

35 randomly allocated.tw. (28558)

36 allocated randomly.tw. (2271)

37 (allocated adj2 random).tw. (788)

38 Single blind\$.tw. (20051)

39 Double blind\$.tw. (177385)

40 ((treble or triple) adj blind\$).tw. (725)

41 placebo\$.tw. (258956)

42 prospective study/ (414841)

43 or/25-42 (1837099)

Intrauterine administration of human chorionic gonadotropin (hCG) for subfertile women undergoing assisted reproduction (Review) 
44 case study/ (51204)

45 case report.tw. (342456)

46 abstract report/ or letter/ (1012507)

47 or/44-46 (1397981)

4843 not 47 (1790343)

4924 and $48(861)$

\section{Appendix 5. PsycINFO search strategy}

\section{OVID Platform}

Searched from 1806 to 9 January 2018

1 exp reproductive technology/ (1682)

2 in vitro fertili?ation.tw. (684)

3 icsi.tw. (68)

4 intracytoplasmic sperm injection\$.tw. (52)

5 (blastocyst adj2 transfer\$).tw. (4)

6 (embryo\$ adj2 transfer\$).tw. (140)

7 or/1-6 (1957)

8 exp Gonadotropic Hormones/ (4096)

9 (Human Chorionic Gonadotrop?in adj7 intrauter\$).tw. (0)

10 (Human Chorionic Gonadotrop?in adj7 uter\$).tw. (0)

11 (intrauter\$ adj7 ?hcg).tw. (0)

12 (uter\$ adj7 ?hcg).tw. (0)

13 or/8-12 (4096)

147 and 13 (8)

\section{Appendix 6. CINAHL search strategy}

\section{EBSCO Platform}

Searched from 1961 to 9 January 2018

\begin{tabular}{|c|c|c|}
\hline \# & Query & Results \\
\hline S15 & S8 AND S14 & 59 \\
\hline S14 & S9 OR S10 OR S11 OR S12 OR S13 & 697 \\
\hline S13 & TX(Chorionic Gonadotrop?in N7 intrauter $\left.{ }^{\star}\right)$ & 1 \\
\hline S12 & TX(Chorionic Gonadotrop?in N7 uter $)$ & 3 \\
\hline S11 & TX(Human Chorionic Gonadotrop?in N7 intrauter ${ }^{\star}$ ) & 0 \\
\hline S10 & TX(Human Chorionic Gonadotrop?in N7 intrauter $\left.{ }^{\star}\right)$ & 1 \\
\hline S9 & (MM "Gonadotropins, Chorionic") & 588 \\
\hline S8 & S1 OR S2 OR S3 OR S4 OR S5 OR S6 OR S7 & 5290 \\
\hline S7 & TX embryo* N3 transfer* & 1159 \\
\hline S6 & TX ovar* N3 hyperstimulat $^{\star}$ & 456 \\
\hline S5 & ${\text { TX } \text { ovari }^{\star} \text { N3 stimulat }}^{\star}$ & 419 \\
\hline S4 & TX IVF or TXICSI & 2204 \\
\hline
\end{tabular}

Intrauterine administration of human chorionic gonadotropin (hCG) for subfertile women undergoing assisted reproduction (Review) 
(Continued)

\begin{tabular}{llc} 
S3 & (MM "Fertilization in Vitro") & 1803 \\
\hline S2 & TX vitro fertilization & 3895 \\
\hline S1 & TX vitro fertilisation & 3895 \\
\hline
\end{tabular}

WHAT'S NEW

\begin{tabular}{lll}
\hline Date & Event & Description \\
\hline 23 October 2018 & Amended & Correction of text in Declarations of interest section \\
\hline
\end{tabular}

\section{HISTORY}

Protocol first published: Issue 2, 2015

Review first published: Issue 5, 2016

\begin{tabular}{lll}
\hline Date & Event & Description \\
\hline 15 June 2018 & New search has been performed & $\begin{array}{l}\text { New study data were added, leading to a change to the conclu- } \\
\text { sions of this review. }\end{array}$ \\
\hline 15 June 2018 & $\begin{array}{l}\text { New citation required and conclusions } \\
\text { have changed }\end{array}$ & $\begin{array}{l}\text { New searches were performed for this major update, and addi- } \\
\text { tional RCTs have contributed data (Dehghani Firouzabadi 2016; } \\
\text { Eskandar 2016; Hosseini 2016; Huang 2016; Mostajeran 2017; } \\
\text { Navali 2016). }\end{array}$ \\
\hline 22 June 2016 & Amended & Links to an analysis and to a figure were inserted. \\
\hline
\end{tabular}

\section{CONTRIBUTIONS OFAUTHORS}

LC and NT performed the literature search, assessed studies for eligibility, and extracted the data.

LC performed the analyses and drafted the review.

NT, NRF, and AC provided feedback and edited the review.

All review authors agree with the final version of the review.

\section{DECLARATIONSOF INTEREST}

LC, NT and AC do not have any conflicts of interest to disclose. NRF has received travel costs or advisory board honoraria from GE Healthcare, Merck Serono and Ferring and provides medico-legal reports for court proceedings. He has shares in two fertility clinics.

\section{SOURCES OF SUPPORT}

\section{Internal sources}

- None, Other.

\section{External sources}

- None, Other. 


\section{DIFFERENCES BETWEEN PROTOCOL AND REVIEW}

We slightly narrowed the Cochrane Gynaecology and Fertility Group Specialised Register search strategy.

We performed a subgroup analysis based on IC-hCG dose to address the heterogeneity.

For outcomes with event rates below $1 \%$, we used the Peto one-step odds ratio (OR) method to calculate the combined outcome with $95 \%$ confidence interval.

If a study included multiple treatment arms receiving different doses of hCG, we split the control group proportionately with the experimental groups to avoid analysing control participants in duplicate.

\section{N DEX TERMS}

\section{Medical Subject Headings (MeSH)}

${ }^{\star}$ Embryo Transfer [adverse effects] [statistics \& numerical data]; Abortion, Spontaneous [epidemiology] [etiology]; Chorionic Gonadotropin [*administration \& dosage]; Embryo Implantation [drug effects]; Infertility, Female [ ${ }^{\star}$ drug therapy]; Live Birth [epidemiology]; Pregnancy Rate; Reproductive Control Agents [ ${ }^{\star}$ administration \& dosage]; Uterus

\section{MeSH check words}

Adult; Female; Humans; Pregnancy 